\title{
Tabularia
}

TABULARIA Sources écrites des mondes normands médiévaux

\section{De Normandie en Angleterre : enquête sur la poétique de trois rouleaux mortuaires}

From Normandy to England: investigating funeral rolls' poetry'

Dalla Normandia all'Inghilterra : indagine sulla poetica di tre rotoli mortuari

\section{Monique Goullet}

\section{(2) OpenEdition}

Journals

Édition électronique

URL : http://journals.openedition.org/tabularia/2782

DOI : $10.4000 /$ tabularia.2782

ISSN : $1630-7364$

Éditeur :

CRAHAM - Centre Michel de Boüard, Presses universitaires de Caen

Référence électronique

Monique Goullet, « De Normandie en Angleterre : enquête sur la poétique de trois rouleaux

mortuaires », Tabularia [En ligne], Autour de Serlon de Bayeux : la poésie normande aux XIe-XIIe siècles, mis en ligne le 20 octobre 2016, consulté le 20 avril 2019. URL : http://journals.openedition.org/ tabularia/2782 ; DOI : 10.4000/tabularia.2782 


\title{
De Normandie en Angleterre: enquête sur la poétique de trois rouleaux mortuaires ${ }^{1}$
}

\author{
From Normandy to England: \\ investigating funeral rolls "poetry"
}

\section{Dalla Normandia all'Inghilterra: indagine sulla poetica di tre rotoli mortuari}

\author{
Monique GouLLET \\ LAMOP-UMR 8589, CNRS/ Université Paris 1 - Panthéon Sorbonne \\ goulletm@orange.fr
}

Résumé:

Du haut Moyen Âge à l'époque moderne, des rouleaux mortuaires circulèrent au sein des réseaux de confraternité. Les mots de condoléances (tituli) étaient recueillis par un porteur (rolliger) qui passait de porte en porte. Trois rouleaux ont été sélectionnés ici pour leur rapport avec les aires normande et anglaise: celui de Bruno le Chartreux, mis en circulation en 1101-1103; celui de Mathilde, abbesse de la Sainte-Trinité de Caen (1113-1114); celui de Vital de Savigny (1123-1124). À cette époque et durant tout le XII ${ }^{\mathrm{e}}$ siècle, c'est le mode poétique qui s'imposa dans les rouleaux. Dans chaque poème la scansion a été rendue visible, en sorte que le lecteur puisse en percevoir les schémas métriques et phoniques. Ces vers, improvisés ou écrits à l'avance, sont les témoins d'une mixité métrique, encore quantitative mais déjà partiellement syllabique et rythmique.

Mots-clés: rouleaux des morts, Normandie, Angleterre, poésie, rhétorique funèbre

\begin{abstract}
:
From the early Middle Ages to the modern era, funeral rolls circulated within confraternity networks. Words of condolence (tituli) were collected by a roll carrier (rolliger) who went from door to door. Three rolls were selected here for their relationship with Norman and English areas: that of Bruno the Carthusian, which circulated in 1101-1103; that of Matilda, abbess of the Holy Trinity of Caen (1113-1114); that of Vitalis of Savigny (1123-1124). At that time and during the whole twelfth century, poetry was the most commun medium in these rolls. In every poem scansion has been made visible, so that the reader can perceive metrical and phonic schemes. These verses, improvised or written in advance, show a metric diversity, yet quantitative but already partly syllabic and rhythmic.
\end{abstract}

Keywords: funeral rolls, Normandy, England, poetry, funeral rhetoric

1. Il me faut remercier Marie-Agnès Avenel pour sa relecture savante et l'astucieuse mise en place des codes métriques. Cette étude a été entreprise à l'occasion du colloque Autour de Serlon de Bayeux: la poésie en Normandie aux $\mathrm{XI}^{e}$-XII ${ }^{e}$ siècles. Colloque international organisé par le Centre Michel de Boüard - Craham (UMR 6273), dans le cadre de la Chaire d'excellence d'Edoardo D'Angelo, Bayeux et Caen, 20-22 mars 2014. On situe généralement le poète Serlon de Bayeux entre 1050 et 1120.

Tabularia «Études», nº 16, 2016, p. 217-278, 20 octobre 2016 http://www.unicaen.fr/mrsh/craham/revue/tabularia/print.php?dossier=dossier15\&file=03goullet.xml 
Riassunto:

Dall'Alto Medioevo fino all'Età moderna hanno circolato all'interno delle istituzioni monastiche dei "rotoli» mortuari. Le parole esprimenti il cordoglio (tituli) venivano raccolte da un portatore (rolliger) che passava di porta in porta. In questo lavoro sono stati scelti tre rotoli, per il loro rapporto con l'area normanna e con l'area inglese: quello di Bruno il Certosino, messo in circolazione nel 1101-1103; quello di Matilde, badessa della SainteTrinité de Caen (1113-1114); e quello di Vitale di Savigny (1123-1124). A quest'epoca, e durante il sec. XII, nei rotoli si impone lo stile poetico. In ogni poema la scansione era resa visibile, in modo che il lettore potesse apprezzarne gli schemi metrici e fonici. Questi versi, improvvisati o scritti in anticipo, sono i testimoni di una sorta di mescolanza metrica, ancora quantitativa ma in parte già sillabica e ritmica.

Parole chiave: rotoli mortuari, Normandia, Inghilterra, poesia, retorica funebre

\section{Les rouleaux mortuaires}

Le Recueil des rouleaux des morts, collection monumentale éditée par le regretté Jean Dufour ${ }^{2}$, est un outil exceptionnel pour caractériser la production poétique funéraire anonyme du XII ${ }^{e}$ siècle. Au sein des réseaux de confraternité, ces rouleaux étaient de puissants moyens d'information et de célébration des défunts grâce au processus du porte-à-porte. Un porte-rouleau (rolliger ou rotulifer) s'annonçait dans chaque établissement, faisait circuler le faire-part de décès - qu'on appelle «lettre» (littera) ou «encyclique», rédigée généralement en prose - et demandait que chacun appose sur le rouleau de parchemin un «titre» (titulus) portant des mots de condoléances. Pour la période qui nous intéresse, c'est-à-dire au XII ${ }^{e}$ siècle, les titres de quelque importance sont tous en vers; les autres se résument à quelques lignes de prières stéréotypées dénuées de visée littéraire, ce qui ne diminue en rien leur intérêt historique et prosopographique.

Cette pratique mortuaire - qui à en croire Baudri de Bourgueil, était devenue une mode un peu fatigante ${ }^{3}$ - nous vaut donc un florilège tout à fait original de vers récoltés au fil des établissements visités par les rolligeri. La production est certes du tout-venant, mais elle s'avère précieuse, en ce sens qu'elle pourrait permettre de confronter les œuvres obscures de versificateurs occasionnels à celles de poètes réputés ${ }^{4}$. Cette production "triviale» est aussi un témoignage fort sur l'enseignement du trivium: un nombre non négligeable de scripteurs se désignent comme des écoliers (scolares, discipuli, membres

2. Dufour (éd.), 2005-2013, vol. I et V. Outre des index très précieux, ce dernier volume offre 150 pages environ de renseignements sur la pratique de ces rouleaux et sur leur intérêt pour l'historien, en particulier dans le champ culturel. Signalons aussi: Dufour, 2003; Goullet, 2010, p. 163-198.

3. Sur Baudri de Bourgueil et les rouleaux des morts, voir ses poèmes édités et traduits par Jean-Yves Tilliette, Baldricus Burgulianus [Baudri de Bourgueil], 1998, vol. I, poèmes nº 17, 18, 22, 72 et 73 .

4. Cette confrontation, hors de portée ici, pourrait faire l'objet d'une recherche passionnante. 
d'une schola); d'autres se nomment: on trouve un Otho iuvenis à Norwich ${ }^{5}$, à York un Benedictus, un Ricardus et un Petrus, qui complètent la grande pièce 41 du rouleau de Mathilde ${ }^{6}$; le Radulphus Cadomensis filius Fulcredi du rouleau de Vital de Savigny serait peut-être le célèbre Raoul de Caen, auteur des Gesta Tancredi, mais rien n'est moins sûr ${ }^{7}$. Dans cette moisson, foisonnante par le nombre mais répétitive et limitée par l'exclusivité de la thématique funéraire, il y a quelques vers talentueux et des perles inattendues, qui dépassent et transgressent la rhétorique mortuaire; ce sont de belles exceptions.

Le centre de cette thématique est le paradoxe chrétien, qui place la vie dans la mort et inversement. On pleure la perte de l'être cher, mais on juge inutile de pleurer puisqu'il sera sauvé, ou tout au moins on en présente la requête à la fin du poème. Le deuil chrétien est fait de pleurs mêlés de joie; ce contraste est un leitmotiv des rouleaux. Celui de Vital de Savigny se prête tout particulièrement à ce topos, car un défunt nommé Vitalis est une mine d'inspiration étymologique; le rédacteur de Saint-Étienne de Caen n'y résiste pas et donne un distique élégiaque: Dicitur a vita Vitalis, sit sibi vita. /Quotque sonat nomen sibi felix compleat $o m e n^{8}$, tandis que le rédacteur rouennais de la pièce 15 donne ce seul distique: Abbas Vitalis, qui vitam semper amavit, / Vivat et eterna luce fruatur. Amen ${ }^{9}$; son collègue, rouennais lui aussi, se montre plus subtil dans la pièce 17 , où de Vitalis il extrait habilement virtus et alis (Abbas Vitalis, virtute nitentibus alis... ad astra volavit $)^{10}$. La rhétorique de l'éloge se déploie dans les trois rouleaux; elle se construit autour du nom des défunts Bruno, Vital ou Mathilde, elle joue avec les sonorités qui en font des rimes ou des refrains, elle use de la pronomination ${ }^{11}$.

La rhétorique funèbre peut virer au réalisme macabre, par exemple dans le titre donné à Saint-Léger de Préaux, où l'on évoque les cadavres qui nourrissent les vers et les corps qui pourrissent ${ }^{12}$; c'est là un registre assez exceptionnel. Plus inattendue est la présence dans le rouleau de Mathilde de plaisanteries grivoises et misogynes comme celles de la Vox scolarium des «écoliers» de la cathédrale de Bath ${ }^{13}$. Leurs consœurs, les nonnes de Shaftesbury, ayant déploré la mort de Mathilde ${ }^{14}$, les scolares de Bath leur répondent par un petit couplet sur la

5. Dufour (éd.), 2005-2013, vol. I, $\mathrm{n}^{\circ}$ 114, 40, p. 412

6. Ibid., vol. I, n ${ }^{\circ} 114,41$, p. 413-415.

7. Ibid., vol. I, no 122, 204, p. 585 (Versus Radulfi, filii Fulcredi Cadomensis) et vol. V, p. 74, sur Raoul de Caen, qui pourrait être identifié avec l'auteur des Gesta Tancredi sur le plan des dates et de l'onomastique, mais difficilement sur le plan littéraire.

8. Ibid., vol. I, $\mathrm{n}^{\circ} 122,2$, p. 524, v. 5-6.

9. Ibid., vol. I, $\mathrm{n}^{\circ} 122,15$, p. 532 , v. 1-2

10. Ibid., vol. I, $\mathrm{n}^{\circ} 122,17$, p. 533 , v. 1-2

11. La pronomination est une figure rhétorique qui remplace le nom propre par un attribut ou qui accumule les attributs après le nom propre, ainsi qu'on peut le lire, par exemple, dans le poème donné par la cathédrale de Bayeux pour Bruno le Chartreux: voir Dufour (éd.), 2005-2013, vol. I, $\mathrm{n}^{\circ} 105,150$, p. 338-339: Strenuus et fortis conservator monachorum, / Providus et mortis fundator coenobiorum, etc.

12. Ibid., $\mathrm{n}^{\circ} 105,148$, v. 6-9, p. 337

13. Ibid., $\mathrm{n}^{\mathrm{o}} 114,28$, p. 409 .

14. Ibid., $\mathrm{n}^{\circ} 114,18$, p. 407 . 
responsabilité d'Ėve, qui aurait sali à jamais toute la gent féminine dans l'épisode de la chute originelle. Cette misogynie, entièrement fondée sur la tradition biblique du péché originel dont Ėve fut l'instigatrice et Adam la victime, n'est pas l'apanage des scholars anglais: le rouleau de Mathilde en est parsemé15; elle est en soi une thématique littéraire, un lieu commun.

Pour donner une idée de la production poétique contenue dans les rouleaux, j'ai choisi d'en analyser trois parmi les plus importants qui ont déjà été cités: celui de Bruno le Chartreux ( $\mathrm{n}^{\circ} 105$, mis en circulation en 1101-1103); celui de Mathilde, abbesse de la Sainte-Trinité de Caen ( $\mathrm{n}^{\circ} 114$, mis en circulation en 1113-1114); celui de Vital de Savigny ( $\mathrm{n}^{\circ}{ }_{122}$, mis en circulation en $\left.1123-1124\right)^{16}$. Ces rouleaux sont donc à la fois contemporains entre eux et passés dans des aires géographiques identiques ou voisines, parmi lesquelles j’ai sélectionné la Normandie et l'Angleterre. Il est inutile de présenter Bruno, fondateur de l'ordre des Chartreux, souvent célébré dans son rouleau comme professeur et ermite. Vital de Savigny, issu d'une grande famille et proche d'un frère utérin de Guillaume le Conquérant, qui lui confia des charges importantes, est le fondateur de l'abbaye normande de Savigny et de nombreux ermitages. Quant à Mathilde, abbesse de la Trinité de Caen, elle reste mal connue, mais ce qui est sûr, c'est qu'elle n'est pas la fille homonyme de Guillaume le Conquérant ${ }^{17}$.

Les trois rouleaux sont dans des états de conservation divers. Celui de Mathilde est l'un des plus impressionnants qui soient. Mesurant vingt mètres de long, il était opisthographe, c'est-à-dire écrit des deux côtés. Il fut conservé jusqu'au XVIII siècle dans le trésor de la Trinité de Caen, où on l'exhuma dans un état pitoyable; il a aujourd'hui disparu, et on ne le connaît plus que par des copies incomplètes du XVII ${ }^{e}$ et du XVIII ${ }^{e}$ siècle, qui ne permettent pas de restituer le document original ${ }^{18}$. Le rouleau de Bruno fut récupéré en 1514 à la Chartreuse de Calabre et envoyé à la Grande-Chartreuse. François Dupuy en donna une édition à Bâle en 1515, avant que le rouleau ne soit détruit en juin 1562 dans la dévastation de la Grande-Chartreuse par les bandes du baron des Adrets; toutes les éditions du rouleau de Bruno remontent donc à Dupuy ${ }^{19}$. Quant au rouleau de Vital, sauf le début, l'original est conservé aux Archives nationales ${ }^{20}$. Les rouleaux les plus prestigieux étaient ornés de peintures: cela fut vraisemblablement le cas de celui de Mathilde. Plusieurs titres font en effet

15. Sur ce thème voir encore Goullet, 2010, p. 180-182.

16. Malheureusement le rouleau mortuaire de l'abbé de Fécamp Guillaume II de Rots, mort le 26 mars 1107 ( $n^{\circ}$ 109) est non seulement perdu, mais connu seulement par le témoignage d'Orderic Vital (Dufour (éd.), 2005-2013, vol. I, p. 361-363). Le moine Alleaume (Adelelmus) aurait, selon Orderic, rassemblé une riche documentation biblique pour rédiger l'encyclique, et celle-ci était si belle que beaucoup pleuraient à sa lecture. C'est le seul témoignage précis que nous ayons de ce rouleau qui, étant donné la personne de l'abbé Guillaume, avait dû aller jusqu'en Angleterre, comme les trois autres rouleaux cités.

17. Sur la question, voir Goullet, 2010, cit. n. 2, p. 170-173.

18. Dufour (éd.), 2005-2013, vol. I, no 114, p. 392-393.

19. Ibid., $\mathrm{n}^{\mathrm{o}} 105$, p. 278-279.

20. A. N., L 966, nº 4. Voir Dufour (éd.), 2005-2013, vol. I, nº 122, p. 514-521. 
allusion à une pictura: le premier vers du titre $\mathrm{n}^{\circ} 2^{21}$, le dernier vers du titre $123^{22}$ et les vers $5-6 \mathrm{du}$ titre $212^{23}$. Le fait que, dès le titre $\mathrm{n}^{\circ} 2$, cette image soit évoquée trois fois en termes proches conduit à penser qu'il s'agit d'une seule et même peinture placée en tête du rouleau, une sorte de frontispice peint, représentant Mathilde au paradis entourée du chœur des vierges selon le titre $\mathrm{n}^{\circ} 212$; l'image peinte (pictura), et la lettre (litura) $)^{24}$ - terme par lequel on peut entendre ici soit la longue lettre-encyclique liminaire ${ }^{25}$, soit une brève inscription (titulus) en forme de «légende» commentant l'image - signifient et certifient (testificante) ensemble la sainteté de Mathilde.

Étant donné la tradition manuscrite et éditoriale de nos trois rouleaux, il est évident que nous n'en lisons pas le texte original: le temps a dû marquer de son empreinte la langue et la métrique; ce facteur, contre lequel nous ne pouvons rien, devra toujours demeurer en toile de fond. Par ailleurs la récolte est inégale: selon le rang du défunt et selon la présence ou l'absence de lettrés dans les établissements visités, lorsque le rolliger frappe à la porte, tantôt il ne recueille qu'une brève formule de prière pour le défunt et pour tous ceux de l'établissement, tantôt, au contraire, des pièces versifiées, qui feront l'objet de notre étude ${ }^{26}$.

\section{Prosodie et métrique}

Pour sensibiliser les lecteurs qui ne seraient pas familiers à la prosodie et à la métrique médiévales, les textes présentés ont été appareillés des notes indispensables et surtout d'aides à la perception des schémas métriques: les dactyles sont figurés en gras, les spondées en romain, des barres verticales simples séparent les pieds; des caractères en exposant indiquent la (ou les) césure(s): un ${ }^{\mathrm{P}}$ marque la césure penthémimère dans l'hexamètre dactylique, un ${ }^{\mathrm{D}}$ suivi d'une barre verticale la césure du pentamètre dans le distique élégiaque. La diérèse est marquée par un ${ }^{\mathrm{D}}$ suivi d'un nombre correspondant à sa place dans le vers. Les coupes plus rares (trihémimère, hepthémimère, $3^{\mathrm{e}}$ trochaïque) sont représentées

21. Ibid., $\mathrm{n}^{\circ} 114,2$, v. 1, p. 400 : Signat pictura quia non est mors nocitura.

22. Ibid., $\mathrm{n}^{\circ} 114,123$, v. 5 , p. 451 : Non scriptura vel pictura rotuli quem bajulas

23. Ibid., $\mathrm{n}^{\circ} 114,212$, v. 5-6, p. 494: Sed cum pictura tibi testificante litura / Felix virgineis gaudes sociata coreis.

24. Le mot litura est ici un équivalent de littera, confusion installée dès l'époque mérovingienne et très bien attestée au XII ${ }^{\mathrm{e}}$ siècle, en particulier en poésie hexamétrique, où, à la différence de littera, le mot litura fournit une finale prosodiquement adéquate. La clausule mandare lituris est employée au sens de mandare litteris («mettre par écrit»), entre autres chez Flodoard, chez Baudri de Bourgueil, dans les Carmina Ratisponensia. Voir Goullet, 2010, cit. n. 2, p. 166-167, avec une traduction française de l'encyclique, p. 168-170.

25. Dufour (éd.), 2005-2013, vol. I, p. 396-399.

26. Le premier volume de la série publiée par Jean Dufour va jusqu'en 1180 et contient essentiellement des pièces versifiées; dans notre corpus sélectif, constitué par les parties normandes et anglaises des trois rouleaux $n^{\circ} 105,114$ et 122, il n'y a aucun texte en prose. À partir du deuxième volume (1181-1399), les tituli s'abrègent considérablement et ne contiennent plus que la liste des défunts, accompagnée ou non de quelques phrases de prière; il n’y a plus que des vers sporadiques. 
de la manière suivante: un ${ }^{\mathrm{T}}$ pour la trihémimère, un ${ }^{\mathrm{H}}$ pour l'hepthémimère et un ${ }^{\mathrm{F}}$ pour la $3^{\mathrm{e}}$ trochaïque ${ }^{27}$. Les rimes internes sont signifiées par de l'italique, et les rimes externes par le souligné.

Toute la production traitée dans les parties normandes et anglaises de nos trois rouleaux relève de la métrique dactylique. Les distiques élégiaques sont minoritaires mais très bien représentés, soit sous forme de poèmes entiers, soit au début, au milieu ou à la fin de poèmes hexamétriques.

Du point de vue prosodique, la poésie médiévale conserve une partie de ses caractères antiques, ou plutôt ses caractères tardo-antiques, que Bède avait rassemblés dans son De re metrica ${ }^{28}$. Au milieu du Moyen Âge, quelques grandes tendances s'accentuent ${ }^{29}$. Le phénomène prosodique le plus important est l'indifférenciation possible de toutes les voyelles, quelle que soit leur place dans le mot ou dans le vers ${ }^{30}$. C'est ainsi que, dans les poèmes des rouleaux, le -o de Bruno (comme aussi dans les finales des verbes de la $\mathrm{r}^{\mathrm{re}}$ personne de l'indicatif), et le - $a$ de Mathildis varient de quantité d'un poème à l'autre et même d'un vers à l'autre; les mots latins d'origine grecque ne prennent pas en compte eux non plus la longueur des voyelles: la $2^{\mathrm{e}}$ syllabe d'ecclesia est toujours brève de même que la $2^{\mathrm{e}}$ syllabe de $(h)$ eremita et $(h)$ eremus; toutes les syllabes de philosophia sont brèves en grec, mais pas ici ${ }^{31}$. En fin de vers, le $i$ accentué de Maria s'allonge pour former le trochée final du vers. Les graphies médiévales étant instables, il suffit d'ajouter ou de retrancher une consonne pour faire varier les quantités: par exemple relligio entraîne de manière factice l'allongement de la syllabe re-, brève par nature; mais religio orthographié avec un seul $l$ peut aussi commencer par un $r e-$ long $^{32}$. En revanche dans le rouleau de Bruno on trouve une occurrence isolée de l'abrègement du préfixe du verbe praebere > prebere; la graphie -e au lieu de ae a-t-elle favorisé la chose ${ }^{33}$ ? Ce n'est pas sûr. Le $e$ de ille s'allonge devant $q u i^{34}$ et l'enclitique -que devant sapientem $^{35}$. À tout cela s'ajoute le fait que devant une césure toutes les syllabes peuvent s'allonger; on parle alors de productio ob caesuram (allongement devant la césure) ${ }^{36}$.

27. La coupe trihémimère, comme son nom l'indique, se situe après le $3^{\mathrm{e}}$ demi-pied, et l'hepthémimère après le $7^{\mathrm{e}}$ demi-pied: Vita bre|vis ${ }^{\mathrm{T}}$ ca|susque le|vis, ${ }^{\mathrm{H}}$ nec $\mid$ spes reme|andi; la coupe dite " $3^{\mathrm{e}}$ trochaïque" se situe au $3^{\mathrm{e}}$ pied, après le trochée $(-\cup)$ : Mathil|di mise|rērě, ${ }^{\mathrm{F}}$ tŭ|ae mise|rere pu|sillae.

28. BÈde LE VÉnÉrable, 1975.

29. Pour tout ce qui suit, voir NORBERG, 1958.

30. Ibid., p. 7-10.

31. Sur ecclesia, eremus / eremita, philosophia, voir $n^{\circ} 105,146$, v. 1, v. 8, v. 16; 155, v. 7; 153, 11.

32. Rouleau $\mathrm{n}^{\circ} 114,42$, p. 415-416, v. 7 .

33. Rouleau $n^{\circ} 105,117$, v. 1: Poscitis | auxili|um, ${ }^{\text {P }}$ prĕbe|at. Voir Norberg, 1958, p. 8-9, qui donne de nombreux exemples, et en particulier adhĕrebo. Ce fait se produit dès l'Antiquité, et il est plus tard développé au contact de la poésie rythmique.

34. Rouleau $\mathrm{n}^{\circ}$ 105, 132, v. 14: Illē | qui bonus | est.

35. Rouleau $\mathrm{n}^{\mathrm{o}} 105,132$, v. 17: Quis tam $\mid$ feli|cem ${ }^{\mathrm{P}}$ no|vit ${ }^{\mathrm{H}}$ at|quē sapi|entem?

36. Ce fait est très courant; on en trouve une occurrence dans le vers cité dans la note précédente: la finale de novit s'allonge devant la césure hephthémimère. 
La métrique médiévale tend ainsi à devenir syllabique et de moins en moins quantitative ${ }^{37}$. L'absence totale d'élision et d'aphérèse dans notre corpus va en ce sens. Autre conséquence de la régression du modèle purement quantitatif: la césure penthémimère s'impose à environ $95 \%$ dans l'hexamètre dactylique ${ }^{38}$, accompagnée du phénomène médiéval par excellence qu'est la rime; on la trouve en fin de vers ${ }^{39}$, mais plus souvent encore en position de rime interne, appelée aussi "léonine ${ }^{40}$. À l'intérieur d'un même poème on peut passer de la rime léonine à la rime finale, ou à des rimes entre fins d'hémistiches, ou encore les cumuler; la rime peut aussi être absente. Les rimes sont tantôt pauvres, tantôt réduites à l'assonance, ou bien riches, voire "très riches", ornées de schémas complexes et suggérant parfois des fins mélodiques, sous l'influence des poèmes rythmiques.

Prenons un exemple. À Saint-Gabriel, prieuré de Fécamp au nord-est de Caen, le titre $\mathrm{n}^{\mathrm{o}} 2$ du rouleau de Mathilde commente une image peinte qui devait vraisemblablement se trouver avant l'encyclique, en tête du rouleau ${ }^{41}$. Hormis les quatre premiers vers et l'antépénultième, qui ont des rimes léonines, les autres hexamètres sont tous holodactyliques et divisés en trois parties (deux fois deux dactyles, plus la clausule hérö̈que). Les deux premières parties du vers riment ensemble, tandis que la fin du vers rime avec la fin du suivant ( $\mathrm{aB} / \mathrm{bbB}$ ). C'est encore là un détournement de l'hexamètre quantitatif vers une métrique syllabique, dans laquelle les coupes tombent après un nombre constant de syllabes et de pieds, contrairement à la métrique classique, où les coupes ne sont pas à la fin d'un mot mais à l'intérieur d'un mot. Le jeu des rimes - fondées sur un petit nombre de sonorités, ce qui provoque un effet obsédant - est une transposition des vers dits trinini salientes ${ }^{42}$, où les rimes internes se trouvent généralement après les coupes trihémimère et hepthémimère ${ }^{43}$; lorsque les rimes se trouvent après un groupe de deux dactyles, on les nomme tripertiti dactilici ${ }^{44}$. Ce système produit un rythme monotone et très rapide, en raison de la longueur de ces vers de 17 syllabes (le nombre le plus élevé qu'on puisse trouver dans un hexamètre) et de la fréquence des syllabes brèves, deux fois plus nombreuses que les longues si l'on excepte le dernier pied:

$$
\begin{aligned}
& \text { 5Non erat| hostia }{ }^{\mathrm{D} 2} \mid \text { quae mori|entia }{ }^{\mathrm{D} 4} \mid \text { morte pi|aret. } \\
& \text { Si foret }\left|{\text { host } i a^{\mathrm{D} 2}}\right| \text { digna per } \mid \text { omnia }{ }^{\mathrm{D} 4} \mid \text { sacrifi|caret }
\end{aligned}
$$

37. Un exemple extrême: Imbu|tus fon $\mid \mathrm{te}^{\mathrm{P}}$ to|tius $\mid$ philoso|phie $\left(\mathrm{n}^{\mathrm{o}} 105,146, \mathrm{v}\right.$. 8): le -e de fonte s'allonge devant la césure (productio ob caesuram); le $i$ de totius est normalement bref mais s'allonge, et selon le modèle grec toutes les syllabes de philosophia devraient être brèves. Ce vers est un quasi-équivalent syllabique de l'hexamètre.

38. On trouve aussi quelques $3^{\mathrm{e}}$ trochaïques, accompagnées ou non d'une trihémimère et/ou hephthémimère, et quelques cas de trihémimère/hephthémimère structurant une rime.

39. On parle alors de versus caudati.

40. La dernière syllabe du premier hémistiche rime avec la dernière syllabe du vers: Poscitis | auxili|um, ${ }^{\mathrm{P}}$ prebe|at quod | Christus in $\mid$ evum $\left(\mathrm{n}^{\circ} 105,117, \mathrm{v} .1\right)$.

41. Dufour (éd.), 2005-2013, vol. I, $\mathrm{n}^{\circ} 114,2$, p. 400.

42. Voir Norberg, 1958, p. 66.

43. C'est le cas du poème $\mathrm{n}^{\circ} 122,12$, v. 1-8.

44. Voir Norberg, 1958 p. 67. 
Hanc homo. $\mid$ Non erat $^{\mathrm{D} 2} \mid$ hic neque ${ }^{\mathrm{D} 3} \mid$ venerat
D4 $\mid$ ut repa|raret
Vel pere $\mid$ untia, ${ }^{\mathrm{D} 2} \mid$ vel $^{\mathrm{P}}$ mori|entia ${ }^{\mathrm{D} 4} \mid$ vivifi|caret.

etc.

On constate ici deux choses: d'une part la structure de la phrase est déconnectée de la structure du vers (ponctuation forte après homo); d'autre part on trouve, contre la métrique classique, des quadrisyllabes et des pentasyllabes en fin de vers. Cette irrégularité favorise l'abus de ce qu'on appelle la «coupe bucolique», que Bède qualifiait de passio: selon lui c'était un inter-mot entre le $4^{\mathrm{e}}$ et le $5^{\mathrm{e}}$ pied, survenant comme une sorte d'accident ou de «hasard», et que les théoriciens médiévaux n'ont jamais interdit. Cet «accident» révèle une faiblesse de nos poètes, qui utilisent souvent des fins de vers formulaires, et qui les «pensent» en quelque sorte comme isolées du reste; le couple penthémimère/bucolique concourt à la monotonie. Or ici c'est bien l'effet recherché, car les deux dernières mesures du vers (rimes finales en -aret) constituent une sorte de refrain monotone qui interfère avec les deux rimes internes en -ntia.

Le poème $\mathrm{n}^{0} 114,11$, Versus cuiusdam neptis suae ${ }^{45}$, fonctionne lui aussi sur un mode quasi musical: le mot final Maria revient dans toutes les fins de vers et fait refrain; les tonalités en $-a$ et $-e$ dominent, surtout dans les seconds hémistiches, et s'accordent avec celle du refrain:

$$
\begin{array}{ll}
\text { Post obi|tum ma|tri || prope | sis, reve|renda } & \text { Ma|ria } \\
\text { Nomine | Mathil|di || pia | sis, bene|dicta } & \text { Ma|ria } \\
\text { Hanc bene | defen|das, || for|tis vir|tute } & \text { Ma|ria }
\end{array}
$$

Ces poèmes écrits dans l'urgence - à moins qu'on les ait préparés d'avance - sont les témoins d'une mixité métrique, encore quantitative mais déjà partiellement syllabique et rythmique.

\section{Établissements visités et inventaire des pièces versifiées ${ }^{46}$}

Dans la liste ci-dessous, qui nomme les établissements normands et anglais dans lesquels ont circulé les trois rouleaux, chacun des titres est accompagné du nombre de vers copiés sur le rouleau; ces vers sont reproduits dans la seconde partie de l'article. Lorsque les vers ne dépassent pas le nombre de deux, je les ai signalés sans les reproduire: dans la liste ils sont annoncés par le sigle $[\boldsymbol{\Delta}]$. Ce sigle est également utilisé pour indiquer les titres qui n’ont produit que de très brèves formules de prières.

\footnotetext{
45. Dufour (éd.), 2005-2013, vol. I, nº 114, 11, p. 405, ici v. 1-3.

46. L'ordre des textes est celui de l'édition de Jean Dufour, qui diffère en partie de l'itinéraire indiqué sur les cartes: voir les rouleaux de Bruno, no 105, p. 701-704, de Mathilde, no 114, p. 705-710, et de Vital, no 122, p. 711-713.
} 


\section{Rouleau de Bruno le Chartreux (no 105)}

\section{Normandie}

Préaux (Saint-Léger), 117, p. 325 (3 hexamètres)

Rouen (cathédrale N.-D.), 146, p. 336-337 (22 hexamètres)

Rouen (Sainte-Trinité[-du-Mont]), 147, p. 337 (9 hexamètres)

Préaux (Saint-Léger), 148, p. 337-338 ( 1 hexamètre +6 distiques +2 hexamètres)

Bayeux (cathédrale N.-D.), 150, p. 338-339 (16 hexamètres)

S.l. [Bayeux] (cathédrale N.-D.), 151, p. 339 (7 hexamètres, Versus scholares eiusdem urbis)

[Bayeux] Saint-Georges, 152, p. 339 (7 hexamètres)

Caen (Saint-Étienne), 153, p. 339 (5 distiques élégiaques)

Cerisy[-la-Forêt] Saint-Vigor, 154, p. 340 [ $\mathbf{\Delta}]$

Coutances (cathédrale N.-D.), 155, p. 340 (7 distiques)

S.l. [Coutances (cathédrale N.-D.) ?], 156, p. 340 (3 distiques, Versus scolares eiusdem urbis)

Le Mont-Saint-Michel, 157, p. 340-341 (6 hexamètres)

\section{Angleterre}

London, Cathedral S. Paul, 121, p. 326 (6 hexamètres)

Canterbury (S. Peter), 128, p. 329 (2 hexamètres [ $\triangle \mathbf{\Delta}]$ )

Bury (S. Edmunds), 129, p. 329 (1 distique élégiaque $[\Delta]$ )

Bury (S. Edmunds), 130, p. 329 (1 distique élégiaque $[\Delta]$ )

Spalding (S. Mary et S. Nicholas), 131, p. 329-330 (14 distiques)

Lincoln (cathedral S. Mary), 132, p. 331 (12 distiques élégiaques)

York (S. Mary), 133, p. 331 (2 distiques élégiaques)

York (S. Mary), 134, p. 331-332 (12 hexamètres)

[York (cathedral S. Peter)], 135, p. 332 (1 distique élégiaque [ $\mathbf{\Delta}$ ])

[York (cathedral S. Peter)], 136, p. 332-333 (10 distiques élégiaques)

Beverley (S. John), no 105, 137, p. 333 (7 hexamètres)

[York (cathedral S. Peter)], 138, p. 333 (12 hexamètres)

[York (cathedral S. Peter)], 139, p. 334 (2 hexamètres [ $\mathbf{\Delta}$ ])

[York (cathedral S. Peter)], 140, p. 334 (1 distique élégiaque [ $\mathbf{\Delta}$ ])

[York (cathedral S. Peter)], 141, p. 334 (14 hexamètres)

[York (cathedral S. Peter)], 142, p. 334-335 (5 distiques élégiaques) 
Chester (S. Peter), 143, p. 335 (5 distiques élégiaques)

[Chester (S. Peter)], 144, p. 335 (12 hexamètres)

Malmesbury (S. Mary, S. Aldhelm), 145, p. 335-336 (18 hexamètres + 1 distique élégiaque)

\section{Rouleau de Mathilde abbesse de la Sainte-Trinité de Caen}

\section{Normandie 1}

Caen (Saint-Étienne), 1, p. 399 (20 hexamètres)

Saint-Gabriel, 2, p. 400 (22 hexamètres)

Sées (Saint-Martin), 4, p. 401 [ $\mathbf{\Delta}]$

Bayeux (cathédrale N.-D), 5, p. 401-402 (7 hexamètres)

Saint-Vigor le Grand, 6, p. 402 [ $\mathbf{\Delta}]$

Fontenay[-le-Marmion] (Saint-Étienne), 7, p. 403 (3 distiques élégiaques)

Beaumont[-en-Auge] (Notre-Dame), 8, p. 403 [ $\triangle$ ]

\section{Angleterre}

Winchester, cathedral (S. Peter, S. Swinthun), 9, p. 403-404 [ $\mathbf{\Delta}$ ]

Winchester (S. Peter, S. Grimbald), 10, p. 404 [ $\mathbf{\Delta}]$

Winchester (S. Mary, S. Edburga), 11, p. 404-405 (8 hexamètres +1 distique élégiaque; 8 hexamètres, Item; 12 hexamètres, Versus cuiusdam neptis suae)

Wherwell, Holy Cross, S. Peter, 12, p. 405-406 [ $\Delta$ ]

Amesbury, S. Mary, S. Melot, 13, p. 406 (2 hexamètres [ $\mathbf{\Delta}]$ )

Salisbury, cathedral (S. Mary), 14, p. $406[\mathbf{\Delta}]$

Wilton, S. Mary, S. Edith, 15, p. 406 [ $\mathbf{\Delta}]$

[Lewes], S. Pancras, 16, p. 406 [

Wilton, 'scola', 17, p. 406-407 (6 hexamètres, Guitoniensis scolae)

Shaftesbury (S. Mary, S. Edward), 18, p. 407 (12 hexamètres)

Milton, S. Mary, S. Samson, 19, p. 407-408 [ $\triangle$ ]

Exeter (Cathedral S. Mary, S. Peter), 20, p. 408 (8 hexamètres)

Burton [upon Trent] (Modwenstow), 21, S. Mary, p. 408 [ $\mathbf{\Delta}$ ]

[Lenton] Holy Trinity, p. 408, 22 [ $\mathbf{\Delta}]$

Exeter (S. Nicholas), 23, p. 408 (3 hexamètres)

Montacute, S. Peter, 24, p. 408 [ $\mathbf{}$ ]

Muchelney, S. Peter, 25, p. 409

http://www.unicaen.fr/mrsh/craham/revue/tabularia/print.php?dossier=dossier15\&file=03goullet.xml 
Glastonbury, S. Mary, 26, p. 409

Bath, cathedral (S. Peter), 27, p. 409 [

[Bath] (scolares), 28, p. 409 (20 hexamètres, Vox scolarium)

Malmesbury, S. Mary, S. Aldhelm, 29, p. 409 [ $\Delta$ ]

Tewkesbury, S. Mary, 30, p. $410[\mathbf{\Delta}]$

Winchcombe, S. Mary, 31, S. Kenelm, p. $410[\mathbf{\Delta}]$

Evesham (S. Mary, S. Egwin), 32, p. 410 (8 hexamètres)

Pershore (S. Mary), 33, p. 410 (4 hexamètres)

York, Holy Trinity, 34, p. 411 [ $\Delta]$

Malvern, S. Mary, S. Michael, 35, p. 411 [ $\mathbf{\Delta}]$

Coventry, cathedral (Holy Trinity, S. Mary), 36, p. $411[\mathbf{\Delta}]$

Tutbury, S. Mary, 37, p. 411 [ $\mathbf{\Delta}$ ]

Blyth, S. Mary, 38, p. 411 [ $\mathbf{\Delta}]$

Selby (S. German), 39, p. 411-412 (7 distiques élégiaques)

Norwich (cathedral Holy Trinity), 40, p. 412 (versus Othonis iuvenis, 6 distiques élégiaques)

York (S. Mary), 41, p. 413-415 (versus Benedicti, 50 hexamètres; versus Ricardi, 14 distiques élégiaques; versus Petri, 8 hexamètres)

York (cathedral S. Peter), 42, p. 415-416 (11 hexamètres)

Lincoln (cathedral S. Mary), 43, p. 416-417 (18 hexamètres)

Crowland, S. Bartholomew, S. Guthlac, 44, p. 417 [

Spalding, S. Mary et S. Nicholas, 45, p. $417[\mathbf{\Delta}]$

S. Benet of Hulme, 46, p. 418 ( 8 hexamètres)

Ramsey (S. Benedict), 47, p. 418-419 (9 hexamètres)

Huntingdon, S. Mary, 48, p. 419 [ $\triangle$ ]

S. Albans, 49, p. 419-420 ( 8 hexamètres +1 distique élégiaque)

London (Westminster, S. Peter), 50, p. 420 (10 hexamètres)

[London] cathedral (S. Paul), 51, p. $420[\mathbf{\Delta}]$

Bermondsey, S. Saviour, 52, p. $421[\Delta]$

Southwark, S. Mary, 53, p. $421[\Delta]$

Rochester, cathedral, 54, p. $421[\Delta]$

Malling, S. Mary, 55, p. $421[\Delta]$

Canterbury, cathedral (Christchurch), 56, p. $421[\Delta]$

[Canterbury], S. Peter, S. Augustine, 57, p. 421 [ $\Delta$ ]

Battle, S. Martin, 58, p. $421[\Delta]$ 
Normandie 2

Troarn, S. Martin, 59, p. 421 (2 hexamètres $[\mathbf{\Delta}])$

Saint-Sauveur[-le-Vicomte], 6o, p. $422[\Delta$

Lisieux (cathédrale Saint-Pierre), 61, p. 422-423 (5 distiques élégiaques; Item 3 distiques élégiaques)

Lisieux (N.-D.[-du Pré]), 62, p. 423-424 (9 hexamètres)

Cormeilles (N.-D.), 64, p. 424-425 [ $\triangle$ ]

Préaux (Saint-Pierre), 65, p. $425[\mathbf{\Delta}]$

Préaux (Saint-Léger), 66, p. 425-426 (10 hexamètres)

Grestain (N.-D.), 67, p. 426 [ $\Delta]$

Le Bec-Hellouin (N.-D.), 68, p. 426-427 (14 hexamètres)

La Croix-Saint-Leufroy, 69, p. 427-428 [ $\triangle$ ]

Jumièges (Saint-Pierre), $70[\mathbf{\Delta}]$

Saint-Wandrille, 71, p. 428-429 (4 hexamètres)

Montivilliers (N.-D.), 72, p. 430 (8 hexamètres)

Fécamp (La Trinité), 73, p. $430[\Delta]$

Longueville[-sur-Scie] (Sainte-Foy), 74, p. $431[\Delta]$

Saint-Victor[-l'Abbaye], 75, p. 431 (4 hexamètres)

Rouen (cathédrale N.-D.), 76, p. 431-432 (4 distiques élégiaques; Item 10 hexamètres)

Rouen (Saint-Amand), 77, p. $432[\Delta]$

Rouen (Saint-Ouen), 78, p. 433 (4 hexamètres)

Rouen (Sainte-Trinité[-du-Mont]), 79, p. 433 ( 5 hexamètres)

Rouen (Saint-Paul), 8o, p. 433-434 (4 hexamètres)

Évreux (Saint-Sauveur), 81, p. 434 [ $\mathbf{\Delta}]$

Évreux (cathédrale N.-D.), 82, p. 434 [ $\mathbf{A}]$

Évreux (Saint-Taurin), 83, p. 434-435 (1 hexamètre $[\boldsymbol{\Delta}])$

Conches[-en-Ouche] (Saint-Pierre), 84, p. 435 (2 distiques élégiaques)

Lyre (N.-D.), 85, p. 435 [

Saint-Évroult, 86, p. 436

Bernay (N.-D. [-de-la-Couture]), 87, p. $436[\mathbf{\Delta}]$

[Saint-Pierre-sur-]Dives (N.-D.), 88, p. 437-438 [ $\mathbf{\Delta}$ ]

Saint-Sever[-Calvados], $89, \mathrm{p} .437$ (3 hexamètres +2 pentamètres)

Cerisy[-la-Forêt] (Saint-Vigor), 9o, p. 437 (7 hexamètres)

Montebourg (N.-D.), 91, p. 438 [ $\mathbf{\Delta}]$

http://www.unicaen.fr/mrsh/craham/revue/tabularia/print.php?dossier=dossier15\&file=03goullet.xml 
Lessay (Sainte-Trinité), 92, p. 438-439 (13 hexamètres)

Bénévent[-l’Abbaye] (Saint-Barthélemy), 93, p. 439 [

Coutances (cathédrale N.-D.), 94, p. 439-440 (18 hexamètres)

Avranches (cathédrale Saint-André), 95, p. 440 (17 hexamètres; Item 14 hexamètres)

Le Mont-Saint-Michel, 96, p. 441-442 (4 hexamètres)

\section{Rouleau de Vital de Savigny}

\section{Normandie}

Lessay (Sainte-Trinité), 1, p. $523[\mathbf{\Delta}]$

Caen (Saint-Étienne), 2, p. 523-524 (6 hexamètres)

Caen (La Trinité), 3, p. 524-525 (10 distiques élégiaques)

Bayeux (cathédrale N.-D.), 4, p. 525-526 (11 hexamètres + 4 distiques élégiaques +9 hexamètres)

Troarn (Saint-Martin), 5, p. $526[\Delta]$

[Saint-Pierre-sur-]Dives (N.-D.), 6, p. 526-527

Lisieux (cathédrale Saint-Pierre), 7, p. 527 (9 hexamètres)

Lisieux (N.-D.[-du Pré]), 8, p. 528 [ $\mathbf{\Delta}]$

Cormeilles (N.-D.), 9, p. $528[\mathbf{\Delta}]$

Préaux (Saint-Pierre), 10, p. 528-529 [ $\mathbf{\Delta}]$

Préaux (Saint-Léger), 11, p. $529[\Delta]$

Grestain (N.-D.), 12, p. 530 (15 hexamètres; Item 29 hexamètres)

Beaumont[-en-Auge] (Notre-Dame), 13, p. 531 [ $\mathbf{\Delta}$ ]

Bernay (N.-D.[-de-la-Couture]), 14, p. 532 [ $\Delta$ ]

Rouen (cathédrale N.-D.), 15, p. 532 (1 distique élégiaque +6 hexamètres)

Rouen (Saint-Ouen), 17, p. 533 (11 hexamètres)

Rouen (Saint-Amand), 18, p. 533-534 [ $\mathbf{\Delta}]$

Rouen, S. Trinité-du-Mont, 19, p. 534 (2 hexamètres $[\mathbf{\Delta}]$ )

Rouen (Saint-Paul), 20, p. 534 (2 distiques élégiaques)

Rouen (N.-D.[-du Pré]), 21, p. 534-535 [ $\mathbf{\Delta}$ ]

Saint-Georges-de-Boscherville, 22, p. 535 [ $\mathbf{\Delta}]$

Jumièges (Saint-Pierre), 23, p. 535 [ $\mathbf{\Delta}]$

Saint-Wandrille, 24, p. 535 [ $\mathbf{\Delta}]$

Lillebonne (clercs), 25, p. 536 [ $\mathbf{\Delta}]$

Montivilliers (N.-D.), 26, p. $536[\Delta]$ 
Fécamp (La Trinité), 27, p. 536 [

Longueville[-sur-Scie] (Sainte-Foy), 28, p. 537 [ $\mathbf{\Delta}$ ]

Longueville[-sur-Scie] (clercs), 29, p. 537 [ $\Delta$ ]

Auffay (N.-D.), 30, p. 537 [ $\mathbf{\Delta}]$

Fécamp (Mathilde recluse, et un clerc), 31 p. 537 [ $\mathbf{\Delta}$ ]

Saint-Victor [l'Abbaye], 32, p. 537 [ $\mathbf{\Delta}]$

\section{Angleterre}

Gloucester (S. Peter), 83, p. 554-555 (2 hexamètres +4 distiques élégiaques)

Tewkesbury (S. Mary), 84, p. 555 [ $\mathbf{\Delta}]$

Evesham (S. Mary), 85, p. 555 (4 hexamètres)

Pershore (S. Mary), 86, p. 555 (3 hexamètres)

Worcester, cathedral (S. Mary), 87, p. 556 [ $\mathbf{\Delta}]$

Coventry, cathedral (Holy Trinity, S. Mary), 88, p. 556 [ $\mathbf{\Delta}]$

Burton [upon Trent] (Modwenstow), S. Mary, 89, p. 556 [ $\mathbf{\Delta}$ ]

Tutbury, S. Mary, 90, p. 556 [ $\mathbf{\Delta}]$

Breedon, canons, 91, p. 556-557 [ $\boldsymbol{\Delta}]$

Blyth, S. Mary, 92, p. 557 [ $\mathbf{\Delta}$ ]

York, S. Mary, 93, p. 557 [ $\mathbf{\Delta}]$

York, cathedral (S. Peter), 94, p. 557 [ $\mathbf{\Delta}]$

Selby, S. German, 95, p. 557 [ $\mathbf{\Delta}]$

Ramsey, S. Benedict, 96, p. 557 [ $\mathbf{\Delta}]$

Norwich, cathedral (Holy Trinity), 97, p. $557[\mathbf{\Delta}]$

Benet of Hulme, 98, p. 558 [ $\Delta$ ]

Barking, S. Mary, S. Ethelburgha, 99, p. 558 [ $\Delta$ ]

[London], Westminster, S. Peter, 100, p. 558 [ $\mathbf{\Delta}]$

Abingdon (S. Mary), 101, p. 558-559 (6 hexamètres; Item alterius 4 hexamètres)

Eynsham (Holy Trinity, S. Mary), 102, p. 559 (4 hexamètres)

Oxford (Holy Trinity, S. Frideswide), 103, p. 559-560 (8 hexamètres)

Rochester, cathedral (S. Andrews), 104, p. 560 [ $\Delta]$

Canterbury, cathedral (Christchurch), 105, p. $560[\Delta]$

[Canterbury], S. Peter, S. Augustine, 106, p. 560 [ $\mathbf{\Delta}$ ]

[Lewes], S. Pancras, 107, p. $560[\Delta]^{47}$

47. À partir du titre 108 apparaissent des établissements qui ne sont ni normands ni anglais; ils ne sont donc pas mentionnés dans cette liste.

http://www.unicaen.fr/mrsh/craham/revue/tabularia/print.php?dossier=dossier15\&file=03goullet.xml 
Cerne, S. Peter, 141, p. $569[\Delta]$

Sherborne, S. Mary, 142, p. 569 [ $\Delta$ ]

Hinckley, S. Mary, 145, p. 570 [ $\Delta$ ]

Battle (S. Martin), 146, p. 570 (7 hexamètres)

[Lenton], Holy Trinity, 147, p. 570 [ $\triangle$ ]

Amesbury, S. Mary et S. Melor, 152, p. 571 [

Wilton (S. Mary, S. Edith), 153, p. 571 (14 hexamètres)

Mulcheney, S. Peter, 154, p. $571[\mathbf{\Delta}]$

Bath, cathedral (S. Peter), 155, p. $572[\Delta]$

Malmesbury, S. Mary, S. Aldhelm, 156, p. 572 [ _ ]

[Barnwell], S. Giles, 157, p. 572 [ $\mathbf{\Delta}$ ]

Belvoir, S. Mary, 158, p. 572 [ $\triangle$ ]

Wymondham, S. Mary, 159, p. $572[\mathbf{\Delta}]$

Pontefract, S. John Evangelist, 160, p. $572[\Delta]$

[Nostell], S. Oswald, 161, p. 573 [

Bridlington, S. Mary, 162, p. 573 (1 distique élégiaque $[\mathbf{\Delta}]$ )

York, Holy Trinity, 163, p. 573 [ $]$

[Great] Bricett, S. Leonard, 164, p. 573 [ $\triangle$ ]

Lincoln, cathedral (S. Mary), 165, p. $573[\mathbf{\Delta}]$

Bardney (S. Oswald), 166, p. 573-574 (20 hexamètres +1 distique élégiaque)

Winchester, cathedral (S. Peter, S. Swithun [Old Minster], 182, p. 579 [ $\mathbf{\Delta}]$ )

Winchester, S. Peter, S. Grimbald [New Minster], 183, p. 579-580 [ $\mathbf{\Delta}$ ]

Winchester, S. Mary et S. Edburga [Nunnaminster], 184, p. 580 (1 hexamètre [ $\Delta$ ])

Romsey, S. Mary, 185, p. 580 [ $\triangle$ ]

Salisbury (Cathedral S. Mary), 186, p. 580-581 (7 hexamètres; Pueriles versus 10 hexamètres +4 distiques élégiaques)

Shaftesbury, S. Mary, S. Edward, 187, p. 581 [ $\mathbf{\Delta}]$

Milton, S. Mary, S. Samson, 188, p. 582

Montacute, S. Peter, 189, p. $582[\Delta]$

Glastonbury, S. Mary, 190, p. $582[\triangle$ ]

Ely, cathedral, 191, p. $582[\Delta]$

Spalding, S. Mary, 192, p. 583 [ $\mathbf{\Delta}$ ]

Crowland, S. Bartholomew et S. Guthlac, 193, p. 583 [ $\triangle$ ]

Thorney, S. Mary et S. Botolph, 194, p. 583 [ $\Delta$ ]

Peterborough, 195, p. $583[\mathbf{\Delta}]$ 
Wallingford, Holy Trinity, 196, p. 583 [

[Bury] S. Edmunds, 197, p. 583 [ $\Delta$ ]

Thetford, S. Mary, 198, p. 583 [ $\triangle$ ]

Eye, S. Peter, 199, p. 584 [ $\triangle$ ]

Colchester, Holy Trinity, 200, p. $584[\mathbf{\Delta}]$

Stratford[-at-Bow], S. Leonard, 201, p. 584 [ $\Delta$ ]

London, Christchurch, 202 (1 distique élégiaque $[\mathbf{\Delta}]$ )

Southwark, S. Mary, 203, p. 584 (4 hexamètres)

London, cathedral (S. Paul), 204, p. 585 (Versus Radulfi, filii Fulcredi Cadomensis, 6 hexamètres +2 distiques élégiaques +12 hexamètres +1 distique élégiaque +1 hexamètre)

Reading, S. Mary, 205, p. 585

Merton, S. Mary, 206, p. 586 [ $\triangle$ ]

Malling, S. Mary, 207, p. $586[\Delta]$

Arundel, S. Nicholas, 208, p. $586[\Delta]$

\section{Bilan}

Dans la partie normande du rouleau $\mathrm{n}^{\circ} 105,11$ titres contiennent des poèmes et un seul titre en est dépourvu; dans la partie anglaise tous les titres contiennent des poèmes, dont 6 (sur un total de 19) ont moins de 3 vers. Dans la première partie normande du rouleau $\mathrm{n}^{\circ} 114$, la moitié des 8 titres contiennent des poèmes; dans la partie anglaise, sur 50 titres on en compte 32 sans poèmes; parmi les 18 poèmes, un seul a moins de 3 vers; dans la seconde partie normande du rouleau, on compte 18 titres en prose et 19 titres avec poèmes (un seul poème a moins de trois vers). Le rouleau $n^{\circ} 122$ compte, pour sa partie normande, un seul titre de moins de 3 vers, 8 titres sont munis de vers sur un total de 22 ; pour sa partie anglaise, 3 titres ont moins de 3 vers chacun, 12 titres ont des poèmes et 57 en sont démunis.

\section{Corpus}

\section{Rouleau de Bruno le Chartreux, $n^{\circ} 105$}

Normandie

Préaux (Saint-Léger), no 105, 117, p. 325

T. Sancti Leodegarii Martyris

1 Poscitis | auxili|um, ${ }^{\mathrm{P}}$ prebe|at quod | Christus in | evum.

Bruno|nis peti|mus ${ }^{\mathrm{P}}$ mi|granti | corpore | Summus 
Quatenus $\mid$ opta|ti ${ }^{\mathrm{P}}$ con $\mid$ cedat $\mid$ munera $\mid$ regni.

Rouen (cathédrale N.-D.), no 105, 146, p. 336-337

\section{T. Sancte Marie Rothomagensis ecclesie}

1 Ecclesi|e sanc|te ${ }^{\mathrm{P}}$ to|tius | lugeat $\mid$ ordo,

Huma|ni gene $\mid$ ris $^{\mathrm{P}}$ flens | irrepa|rabile | damnum

Mundo $\mid$ deces $\mid$ sit $^{\mathrm{P}}$ mun|dani| victor ho|noris

Bruno pa|ter, sanc $\mid \mathrm{te}^{\mathrm{P}}$ fun|dator $\mid$ relligi|onis;

5Cuius | tanta pi|am ${ }^{\mathrm{P}}$ vi| $\operatorname{tam}^{\mathrm{H}}$ com $\mid$ mendat ho|nestas,

Ut sit e|um cui|quam ${ }^{\mathrm{P}}$ non $\mid$ equipa|rare po|testas.

Ipse fu|it sapi|ens, ${ }^{\mathrm{P}}$ vir | nobilis, | indole | fulgens,

Imbu|tus fon|te ${ }^{\mathrm{P}}$ to|tius $\mid$ philoso|phie;

In quo | cum vir|tus ${ }^{\mathrm{P}}$ probi|tatis | viva ni|teret,

10 Glorifi|cos fas $\mid$ ces, ${ }^{\mathrm{P}}$ qua |promeru|isse va|leret,

Procul|tator o|pum, ${ }^{\mathrm{P}}$ cunc|torum | spretor ho|norum,

Et mun|di stul| $\operatorname{tam}^{\mathrm{P}}$ pede $\mid$ contulit $\mid$ ambiti|onem

Et studi $\mid$ o sanc $\mid \operatorname{tam}^{\mathrm{P}}$ fun|davit $\mid$ relligi| onem.

Mundum | decli|nans, ${ }^{\mathrm{P}}$ mun|di ${ }^{\mathrm{H}}$ sub|limia | vitans

15Ele|git poti|us ${ }^{\mathrm{P}}$ pri|vata | degere | vita.

Sed quam|vis humi|lis ${ }^{\mathrm{P}}$ cla| $\mid$ rus $^{\mathrm{H}}$ meri|tis ere|mita,

Cuius | magnifi|ce ${ }^{\mathrm{P}}$ quoni|am seri|em pie|tatis

Nemo re|ferre va|let, ${ }^{\mathrm{P}}$ ac|tusve pi|e boni|tatis,

His pre|termis $\mid$ sis, ${ }^{\mathrm{P}}$ preci|bus nos | invigi|lemus,

20 Atque Pa|trem sum $\mid$ mum $^{\mathrm{P}}$ de|vota | mente ro|gemus,

Ut pater | eximi|us ${ }^{\mathrm{P}}$ vi|tali | luce fru|atur,

Iustus et $\mid$ agmini|bus ${ }^{\mathrm{P}}$ ius $\mid$ torum $\mid$ consoci|etur.

\section{Rouen (Sainte-Trinité[-du-Mont]), no 105, 147, p. 337}

Rothomagi Montis titulus Sancte Trinitatis

1Quamvis | mens homi|nis ${ }^{\mathrm{P}}$ nes|cit dis|cernere, | finis

Quis sit | cuncto|rum; ${ }^{\mathrm{P}}$ sed | fons et o|rigo bo|norum,

Cui patet $\mid$ occul|tum, ${ }^{\mathrm{P}}$ nec | quid di|mittit in|ultum,

Nos tamen, | ut mo|res ${ }^{\mathrm{P}}$ de|signant | exteri|ores,

5Dulcis et $\mid$ emeri|te ${ }^{\mathrm{P}}$ pia $\mid$ consum|matio | vite, 
Credimus, | ad supe $\mid \operatorname{ros}^{\mathrm{P}}$ quos | vester | transiit | heros.

Que vos $\mid$ ora $\mid$ tis $^{\mathrm{P}}$ de $\mid$ votis $\mid$ concele|bratis,

Hec ea|dem nos $\mid$ tris $^{\mathrm{P}}$ ex|poscimus | ut faci|atis,

Ut vi|vant Chris|to, ${ }^{\mathrm{P}}$ quos | carcere | solvit ab | isto.

Préaux (Saint-Léger), no 105, 148, p. 337-338

\section{T. Sancti Leodegarii}

1 Hic Leo $\mid$ degari| $\mathbf{i}^{\mathrm{P}}$ titu|lus ${ }^{\mathrm{H}}$ des $\mid$ cribitur $\mid$ almi,

A faci|e mor $\mid$ tis $^{\mathrm{P}}$ nequit | ullus | sistere | fortis,

Sed fu|mo simi|lis ${ }^{\mathrm{D}}$ | vita vi|ri steri|lis,

Labitur | ut ven $\mid$ tus $^{\mathrm{P}}$ pul|cherrima $\mid$ nata iu|ventus.

5Eque | tollun $\mid$ tur $^{\mathrm{D}} \mid$ pessimus | atque bo|nus.

Omnes $\mid$ morte ru|unt ${ }^{\mathrm{P}}$ ter|ramque ca|davera $\mid$ querunt

In nihi|lum veni|unt, ${ }^{\mathrm{D}}$ | vermibus | esca flu|unt.

Corpora $\mid$ putres $\mid$ cunt, ${ }^{\mathrm{P}}$ que | viva su|perba fu|erunt,

Horrida | fit ca|ro, ${ }^{\mathrm{D}} \mid$ mortua $\mid$ cuncta ca|ro.

10Mox homo | cum mori|tur, ${ }^{\mathrm{P}}$ sta|tim sua |fossa pa|ratur,

Charus | post nul|lus ${ }^{\mathrm{D}}$ | curat ha|bere su|us,

Sed soci|at cine $\mid \mathrm{ri}^{\mathrm{P}}$ iam $\mid$ fetens $\mid$ corpus a|mici.

O quam | vanus a|mor, ${ }^{\mathrm{D}}$ | cum cadit | omnis ho|nor!

Bruno|ni dedi|mus, ${ }^{\mathrm{P}}$ quod | debitus | exigit | usus,

15 Vos et $\mathrm{i} \mid$ dem nos $\mid$ tris $^{\mathrm{D}} \mid$ per|solvite $\mid$ subtitu|latis.

Bayeux (cathédrale N.-D), no 105, 150, p. 338-339

T. Sancte Marie Bajocensis ecclesie

1 Strenuus $\mid$ et for $\mid$ tis $^{\mathrm{P}}$ con|serva|tor mona|chorum,

Providus | et mor|tis, ${ }^{\mathrm{P}}$ fun|dator | coenobi|orum

Sancto|rumque pa|trum ${ }^{\mathrm{P}}$ pius | et cle|mens imi|tator,

Sola|men fra|trum, ${ }^{\mathrm{P}}$ sanc|te vir|tutis a|mator,

5 Morum | correc| $\mid$ tor $^{\mathrm{P}}$ ius|tus, pia $\mid$ spes mise|rorum,

Iustici|e rec|tor, ${ }^{\mathrm{P}}$ cus|tos et u|bique bo|norum,

Moribus | orna|tus, ${ }^{\mathrm{P}}$ vas | et ple|num pie|tatis,

Fortiter $\mid$ arma|tus ${ }^{\mathrm{P}}$ clype $\mid$ o ve|re probi|tatis,

Huma|ne Bru|no ${ }^{\mathrm{P}}$ subi|it ius $\mid$ condici|onis, 
10 Cetibus $\mid$ angeli|ce ${ }^{\mathrm{P}}$ con|iungen|dus legi|onis.

Non ibi | rapto|res ${ }^{\mathrm{P}}$ habi|tant, nec | turba re|bellis

Nec pra|vi mo|res ${ }^{\mathrm{P}}$ ibi $\mid$ sunt, sed | quisque fi|delis.

Quo nobis $\mid$ adi $\mid$ tum $^{\mathrm{P}}$ tribu|at rex $\mid$ perpetu|alis,

Mortis | post obi|tum ${ }^{\mathrm{P}}$ pro|tectio | spiritu|alis.

15Spiritus | hic sum $\mid \mathrm{ma}^{\mathrm{P}}$ Bru|nonis | pace fru|atur

Luceque $\mid$ splendiflu| $\mathbf{a}^{\mathrm{P}}$ pie $\mid$ tate $\mathrm{De} \mid \mathrm{i}$ poti|atur.

S.l. Bayeux (cathédrale N.-D), no 105, 151, p. 339

Versus scholares eiusdem urbis

1Hic dolor, | o Bru|no, ${ }^{\mathrm{P}}$ plus | quam pro|cessit ab | uno;

Unde do|let, plan|git, ${ }^{\mathrm{P}}$ quam | mors tua | funditus | angit!

Non lachry|mando pa|rum ${ }^{\mathrm{P}}$ com $\mid$ munis | turba scho|larum

Atque ge|nu pro| no $^{\mathrm{P}}$ ta|li vidu|ata pa|trono

5 Pro meri|to dan $\mid$ do $^{\mathrm{P}}$ dif|fundit | vota pre|cando

Et re|gem pos $\mid$ cit, ${ }^{\mathrm{P}}$ qui $\mid$ cuncta la|tentia $\mid$ noscit,

Ut tibi $\mid$ det vi|tam, ${ }^{\mathrm{P}}$ te $\mid$ suscipi|ens ere|mitam.

Bayeux (Saint-Georges), no 105, 152, p. 339

T. Sancti Georgii eiusdem urbis

1 Flos ere $\mid$ mita $\mid$ rum, ${ }^{\mathrm{P}}$ lu|men mi|rabile, | clarum

Sydus, | Bruno, pa|trum ${ }^{\mathrm{P}}$ vigor, | ordo, | regula | fratrum

Exem|plarque vi $\mid \mathrm{e}^{\mathrm{P}}$ ce|lestis | fonsque so|phie,

Has teti|git me $\mid$ tas, ${ }^{\mathrm{P}}$ quibus $\mid$ omnis $\mid$ clauditur $\mid$ etas.

5In nume $\mid$ ro fra| trum $^{\mathrm{P}}$ te $\mid$ scripsimus, | optime | patrum,

Sedula $\mid$ devo $\mid \mathrm{te}^{\mathrm{P}}$ fun|dendo pre|camina $\mid$ pro te,

Ut pie $\mid$ tate De $\mid \mathbf{i}^{\mathrm{P}}$ tibi $\mid$ detur $\mid$ pars requi|ei.

Caen (Saint-Étienne), nº 105, 153, p. 339

T. Sancti Stephani ad eundem

1Si vel | per gemi|tum ${ }^{\mathrm{P}}$ vel | per lacry|mas dare | vitam

Hic mun|dus pos $\mid$ set, ${ }^{\mathrm{D}} \mid$ mundus ${ }^{48}$ u|trunque da|ret,

Ut sibi $\mid$ subla|tum ${ }^{\mathrm{P}}$ semel $\mid$ elice|ret redi|vivum

48. J'ai corrigé mundusque en mundus. 
Bruno|nem, mise $\mid$ ris $^{\mathrm{D}}$ | spem, decus, | auxili|um,

5Qui sec|tans ere|mum ${ }^{\mathrm{P}}$ propri|amque cru|cem baiu|lando

Actu $\mid$ comple $\mid$ vit, ${ }^{\mathrm{D}}$ | ore quod $\mid$ edocu|it,

Remigi|umque te| nens $^{\mathrm{P}}$ fide|i spem | fixit in | astris,

Et rate $\mid$ feli $\left|\mathbf{c i}^{\mathrm{D}}\right|$ iam mare $\mid$ transili|it.

Et quia $\mid$ non per $\mid \mathrm{se}^{\mathrm{P}}$ va|let ullus $\mid$ posse be|ari,

10Hunc im $\mid$ mensa De $\mid \mathbf{i}^{\mathrm{D}}$ | gratia | iustifi|cet.

Coutances (cathédrale N.-D.), no 105, 155, p. 340

T. Sancte Marie Constantiensis ecclesie

1Christus, | docto|rum ${ }^{\mathrm{P}}$ doc|tor, fons | atque bo|norum,

Donet | Bruno| $\mid$ ni $^{\mathrm{D}}$ | quo meru|ere bo|ni.

Esse De|um ve|rum ${ }^{\mathrm{P}}$ sequi|tur non | esse se|verum,

Huic igi|tur placi|dus ${ }^{\mathrm{D}}$ | sit sine | fine De|us.

5Nam genus | huma|num, ${ }^{\mathrm{P}}$ con|fectum | crimine | vanum,

Iste pa|ter docu|it ${ }^{\mathrm{D}} \mid$ quam meli|us potu|it,

Christi $\mid$ manda|tum ${ }^{\mathrm{P}}$ pan|dens, dare $\mid$ munus a|matum

Servan $\mid$ ti vi|$\left|\operatorname{tam}^{\mathrm{D}}\right|$ dat quia $\mid$ perpetu|am.

Nec so|lum ver|bis, ${ }^{\mathrm{P}}$ ut $\mid$ durus $\mid$ doctor, a|cerbis

10 Perdocet, | ast fac|tis ${ }^{\mathrm{D}}$ | persequi|tur propri|is.

Perpetu $\mid \mathrm{a}$ vi $\mid \mathrm{ta}^{\mathrm{P}}$ cur $\mid$ non caret $\mid$ hic ere $\mid$ mita,

Est quoni|am ve| rus $^{\mathrm{D}}$ | solum in | orbe De|us.

Quod peti|tis ves|tris, ${ }^{\mathrm{P}}$ hoc $\mid$ Christus | det quoque | nostris,

Insimul $\mid$ ut soci $\left|\mathbf{i}^{\mathrm{D}}\right|$ sint sine $\mid<$ labe $>^{49} \mathrm{De} \mid \mathbf{i}$.

S.l. [Coutances (cathédrale N.-D.)], n⿳0 105, 156, p. 340

Versus scolares eiusdem urbis

1Bruno, | multo|rum ${ }^{\mathrm{P}}$ pre|ceptor | grammati|corum,

Cunctis $\mid$ corpore $\left|\mathrm{um}^{\mathrm{D}}\right|$ nunciat $\mid$ interi|tum.

Grammati|cus, rhe|tor, ${ }^{\mathrm{P}}$ dia|lecticus | astrolo|gusque

Effuge|rent mor|tem, ${ }^{\mathrm{D}}$ | si fugi|enda fo|ret.

5Sed quia $\mid$ mors nul|la ${ }^{\mathrm{P}}$ nequit $\mid$ aufer|ri medi|cina,

Semper $\mid$ quisque su| um $^{\mathrm{D}} \mid$ cogitet $\mid$ interi|tum.

49. Le pentamètre est incomplet, j'ai restitué <labe>.

http://www.unicaen.fr/mrsh/craham/revue/tabularia/print.php?dossier=dossier15\&file=03goullet.xml 
Le Mont-Saint-Michel, no 105, 157, p. 340-341

T(itulus) Sancti Michaelis de periculo maris

1 Mira De|i vir|tus ${ }^{\mathrm{P}}$ mi|randos | perficit | actus.

Quosdam | iustifi|cat, ${ }^{\mathrm{P}}$ quos $\mid$ dam de $\mid$ morte re|pulsat.

Quos vult, | igno|rat, ${ }^{\mathrm{P}}$ quos | vult, soli|de sibi | firmat;

In quibus | hic Bru|no, ${ }^{\mathrm{P}}$ vir $\mid$ relligi|onis a|mator,

5Colligi|tur, capi|tur, ${ }^{\mathrm{P}}$ fe|liciter | annume|ratur,

Huius | sicut o|pus ${ }^{\mathrm{P}}$ rotu|li con|testifi|catur.

\section{Angleterre}

London, Cathedral S. Paul, no 105, 121, p. 326

1 Tanti | fama vi|ri ${ }^{\mathrm{P}}$ dif|fusa sub | ultima | mundi

Excitat $|\operatorname{arden}|$ tes $^{\mathrm{P}}$ in $\mid$ digna pre $\mid$ camina $\mid$ mentes,

Et licet, | ut qui|mus, ${ }^{\mathrm{P}}$ com|plere pe|tita ve|limus,

Vota pro|bato|rum ${ }^{\mathrm{P}}$ pas $\mid \operatorname{sim}^{\mathrm{H}}$ pro|missa vi|rorum

5 Nos pro | posse mo|nent, ${ }^{\mathrm{P}}$ Domi|num ${ }^{\mathrm{H}}$ memo|rare Bru|nonem

Deces $\mid$ sumque su $\mid \mathrm{i}^{\mathrm{P}}$ mis $\mid$ sis $^{\mathrm{H}}$ cele $\mid$ brare quot $\mid$ annis.

Spalding (S. Mary et S. Nicholas), no 105, 131, p. 329-330

T. Sancte Marie Spalinge, ecclesie S. Nicolai Andegavensis

1 In mun|do ruti|lat ${ }^{\mathrm{P}}$ so|lis iubar | et ruti|lando

Transit et $\mid$ exce $\mid$ dit $^{\mathrm{D}}$ | sidera | clara po|li;

Sic et $\mid$ Bruno|nis ${ }^{\mathrm{P}}$ sapi|entia | tanta re|fulsit,

Inter $\mid$ Franco $\mid$ rum $^{\mathrm{D}}$ | sidera $\mid$ solus ut $\mid$ hic

5Esset | cuncto|rum ${ }^{\mathrm{P}}$ flos | et fons | philoso|phorum.

Flos speci|osus e|rat ${ }^{\mathrm{D}}$ | fonsque pro|fundus e|rat.

Ex hoc $\mid$ mana $\mid$ vit $^{\mathrm{P}}$ sapi|entia $\mid$ tanta per $\mid$ orbem,

Ut quos $\mid$ imbue $\mid$ ret, ${ }^{\mathrm{D}} \mid$ philoso|phos face|ret.

Splendor $\mid$ sermo $\mid$ nis $^{\mathrm{P}}$ fuit $\mid$ et lux $\mid$ relligi|onis,

10 Eius $\mid$ relligi $\left|\mathbf{o}^{\mathrm{D}}\right|$ scitur $\mathbf{u} \mid$ bique sa|tis;

Eius | doctri| na $^{\mathrm{P}}$ sunt $\mid$ facti | tot sapi|entes,

Quos mea $\mid$ mens nes $\mid$ cit $^{\mathrm{D}}$ | et mea $\mid$ penna ta|cet;

Eius in $\mid$ exem $\mid$ plo $^{\mathrm{P}}$ sunt $\mid$ multi | relligi|osi

Et sec|tato|res ${ }^{\mathrm{D}}$ | discipu|lique cru|cis. 
15Ipse cru|cem Chris $\mid \mathbf{t i}^{\mathrm{P}}$ tulit $\mid$ et sua $\mid$ cuncta re|liquit,

Seque ne $\mid$ gando si $\left|b i^{\mathrm{D}}\right|$ profuit $\mid$ ipse si|bi.

Dives, | famo|sus, ${ }^{\mathrm{P}}$ fa|cundus ${ }^{\mathrm{P}}$ et gene|rosus

In mun|do nitu|it, ${ }^{\mathrm{D}} \mid$ sed sibi $\mid$ displicu|it.

Mundus et $\mid$ ipsi|us ${ }^{\mathrm{P}}$ res $\mid$ et mun|dana po|testas

20Non sibi | chara fu|it, ${ }^{\mathrm{D}}$ | omnibus | abstinu|it.

Nullus a|mor vel ho|nor ${ }^{\mathrm{P}}$ nocu|ere De|um siti|enti;

Sed que|rendo De|um, ${ }^{\mathrm{D}} \mid$ fugit in $\mid$ exili|um.

Exul e|rat patri|e, ${ }^{\mathrm{P}}$ loca | presidi|umque Ma|rie

Obtinu|it, cu|ius ${ }^{\mathrm{D}}$ | filius | ipse fu|it.

25Felix | exul e|rat, ${ }^{\mathrm{P}}$ quem | Virgo Ma|ria re|cepit.

Hec sibi | refugi|um ${ }^{\mathrm{D}}$ | prestet et | auxili|um,

Fiat e|i por $\mid$ tus, ${ }^{\mathrm{P}} \mathrm{cu} \mid$ ius fuit | integer | ortus

Filius | eter|nus, ${ }^{\mathrm{D}} \mid$ sit tibi $\mid$ certa sa|lus.

Lincoln (cathedral S. Mary), $\mathrm{n}^{\circ}$ 105, 132, p. 331

T. Sancte Marie ecclesie Lincolniensis

1 Transis|ti mun $\mid \mathrm{do}^{\mathrm{P}}$ se|curus, | maxime | Bruno,

Quem quia $\mid$ sprevis|ti, ${ }^{\mathrm{D}} \mid$ ni nocu|isse pu|to.

Te volu|it re|bus ${ }^{\mathrm{P}}$ fal|lacibus | illaque|are,

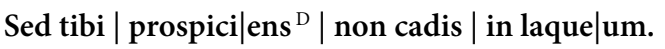

5Obtulit | iste qui|dem, ${ }^{\mathrm{P}}$ que | pronus | stultus a|dorat,

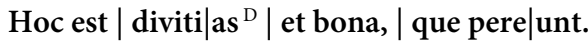

Et quia $\mid$ nemo po|test ${ }^{\mathrm{P}}$ ho|rum splen|dore be|ari,

Quippe ni|hil pro|sunt, ${ }^{\mathrm{D}} \mid$ sed nimis $\mid$ offici|unt,

Omnibus | adiec|tis, ${ }^{\mathrm{P}}$ ere|mum le|tus peti|isti,

10 Hoc so|lum cupi|ens ${ }^{\mathrm{D}} \mid$ posse pla|cere De|o.

Nec bona $\mid$ fama vi|ri ${ }^{\mathrm{P}}$ latu|it dis|persa per | orbem,

Sed vaga $\mid$ permul| tos $^{\mathrm{D}} \mid$ commonu|it popu|los.

Profuit $\mid$ et mul|tis ${ }^{p}$ dans exem|plum famu|landi

Ille, | qui bonus | est, ${ }^{\mathrm{D}}$ | qui Domi|nus Deus | est.

15 Hoc igi|tur meli|us ${ }^{\mathrm{p}}$ qui ho|mo pos|set medi|tari?

Ista sa|lus mul|tis ${ }^{\mathrm{D}} \mid$ profuit $\mid$ atque ti|bi 
Quis tam $\mid$ feli|cem ${ }^{\mathrm{P}}$ no|vit ${ }^{\mathrm{H}}$ at|que sapi|entem?

Non no|vi ta|lem ${ }^{\mathrm{D}}$ | nec tibi | consimi|lem.

In te $\mid$ reve $\mid \mathbf{r a}^{\mathrm{P}}$ patu|it ${ }^{\mathrm{H}}$ sapi|entia | vera,

20Te pie|tas Domi| $\mid$ ni $^{\mathrm{D}}$ | nutriit | et docu|it.

Hunc e|ligi|sti ${ }^{\mathrm{P}}$ pre $\mid$ cunctis, | hunc et a|masti,

Quem qui|cunque co|lit, ${ }^{\mathrm{D}}$ | non colu|isse pu|det.

Sit tibi | lux, patri|a, ${ }^{\mathrm{P}}$ requi|es et | gloria | parta,

Cui ser|vire fu| $\mid$ it $^{\mathrm{D}}$ | gloria $\mid$ sola ti|bi.

York (S. Mary), no 105, 133, p. 331-332

\section{T. Beate Marie Eboracensis}

1 Filius | ecclesi|ae ${ }^{\mathrm{P}}$ mor|tali | carne so|lutus,

Bruno | vir sa|piens, ${ }^{\mathrm{D}}$ | quod sua | facta pro|bant,

Non est | ploran|dus, ${ }^{\mathrm{P}}$ quia | numquam | iam rui|turus,

Spes, amor | atque fi|des, ${ }^{\mathrm{D}}$ | quod meru|ere, te|net.

York (S. Mary), no 105, 134, p. 331-332

Alius eiusdem

$1 \mathrm{O}$ si | voce re $\left|\mathrm{i}^{\mathrm{P}} \mathrm{di}\right|$ vini | pompa tro|phei

Posset | lauda|ri, ${ }^{\mathrm{P}}$ vel | plene | notifi|cari!

Sed quia $\mid$ defice $\mid$ ret, ${ }^{\mathrm{P}}$ nisi $\mid$ se mea | vox cohi|beret,

Quod mihi | velle da|tur, ${ }^{\mathrm{P}}$ per|fecte | posse ne|gatur.

5Sed tamen $\mid$ absque mo|ra ${ }^{\mathrm{P}}$ sur $\mid$ gat mea $\mid$ per mare | prora,

Ut, si | fas de|tur, ${ }^{\mathrm{P}}$ Chris|tum lau|dando pre|cetur.

Laus tibi, | summe Pa|ter, ${ }^{\mathrm{P}}$ cui | subditur | inferus | ater,

Laus tibi, | laus, Chris|te, ${ }^{\mathrm{P}}$ cui | subiacet | iste.

Celes $\mid$ tis do $\mid \mathrm{ni}^{\mathrm{P}}$ da $\mid$ premia, | queso, Bru|noni;

10Ut tibi, | qui vix|it, ${ }^{\mathrm{P}}$ qui | mundo | se cruci|fixit,

Vivat in $\mid$ eter $\mid$ num ${ }^{\mathrm{P}}$ nec $\mid$ mortis $\mid$ tangat $\mathrm{A} \mid$ vernum.

Quod peti|tis vo $\mid$ bis, ${ }^{\mathrm{P}}$ im $\mid$ pendite $\mid$ mutuo $\mid$ nobis.

[York (cathedral S. Peter)], no 105, 136, p. 332-333

Alius titulus eiusdem

1 Fama pri|us no|bis ${ }^{\mathrm{P}}$ retu|lit, quam | litera | vestra,

Non de $\mid$ morte qui|dem, ${ }^{\mathrm{D}}$ | sed boni|tate vi|ri. 
Gemma do|mus Domi|ni ${ }^{\mathrm{P}}$ Bru|no fuit | atque co|lumna,

Et fide $\mid$ i ve $\mid$ rus $^{\mathrm{D}}$ | cultor a|postoli|ce.

5 Vera fi|des e|ius, ${ }^{\mathrm{P}}$ vir|tutibus | edifi|cata,

Ethere $\mid$ as e $\mid$ des $^{\mathrm{D}} \mid$ edifi|cavit e|i.

Gloria, | diviti|e, ${ }^{\mathrm{P}}$ per|sona, sci|entia | rerum

Illi | clara sa|tis, ${ }^{\mathrm{D}} \mid$ sed pede $\mid$ pressit e|a.

Sunt que | dant homi $\mid$ ni $^{\mathrm{P}}$ post $\mid$ mortem | vivere | posse,

10 Nec tamen $\mid$ hec pos $\mid$ sunt $^{\mathrm{D}} \mid$ morte ca|rere da|re.

Mors premit $\mid$ omne ca|put; ${ }^{\mathrm{P}}$ que | si cui | parcere | posset,

Certe $\mid$ Bruno $\mid$ ni $^{\mathrm{D}} \mid$ parcere $\mid$ debue|rat.

Mors bona, | mors fe $\mid$ lix, ${ }^{\mathrm{P}}$ si | mors est | illa vo|canda,

Quam sequi|tur requi|es ${ }^{\mathrm{D}}$ | vitaque | perpetu|a.

15Si quid e $\mid$ i ter $\mid$ rena ${ }^{\mathrm{F}}$ de $\mid$ dit $^{\mathrm{H}}$ con|tagio $\mid$ sordis

(Nam sine $\mid$ pecca $\mid$ to $^{\mathrm{D}} \mid$ vivere $\mid$ nemo po|test)

Abluat | illa De|us, ${ }^{\mathrm{P}}$ qui tri|nus vi|vit et u|nus

Et det e|i requi|em, ${ }^{\mathrm{D}} \mid$ dicat et $\mid$ omnis $\mathrm{A} \mid$ men.

Quod peti|tis ves $\mid$ tris $^{\mathrm{P}}$ iam $\mid$ fecimus $\mid$ et faci|emus,

20Et peti|mus, nos|tris ${ }^{\mathrm{D}}$ | ut faci|atis i|dem.

Beverley (S. John), nº 105, 137, p. 333

T. sancti Iohannis archiepiscopi Beverlacensis ecclesie

1Bruno, | quod spre|vit ${ }^{\mathrm{P}}$ vi|vens, in | morte re|liquit.

Nam mun|dum fu|git ${ }^{\mathrm{P}}$ mun|danaque | vilia | dixit.

Christo | servi|vit, ${ }^{\mathrm{P}}$ qua|re ce|lum pene|travit.

Ipsi|us ta|les ${ }^{\mathrm{P}}$ trans|mittere $\mid$ sed quia | fratres

5 Chartam | fece|runt, ${ }^{\mathrm{P}}$ nos|tri domi|ni volu|erunt

Pro famu|lo tan $\mid$ to ${ }^{\mathrm{P}}$ pos $\mid$ tremo | tempore | functo

Offici|um ser|vis ${ }^{\mathrm{P}}$ gra|tum per|solvere $\mid$ cunctis.

[York (cathedral S. Peter)], no 105, 138, p. 333-334

Item Ricardus ad eundem

1Qui ${ }^{50} \mathrm{ca} \mid$ su mor|tis, ${ }^{\mathrm{P}}$ sub|lato | patre, do|letis,

Ponite | tristici|am, ${ }^{\mathrm{P}}$ manet $\mid$ omnes | exitus | idem ${ }^{51}$.

50. J’ai corrigé quia en qui, pour des raisons évidentes de métrique et de sens (qui... doletis).

51. Les finales -am et -em, qui se sont nasalisées au Moyen Âge, peuvent rimer entre elles. 
Hinc est | pensan|dum, ${ }^{\mathrm{P}}$ quia | mundi | gloria | fenum,

Ad tem|pus flo|ret, ${ }^{\mathrm{P}}$ flo|rens per|transit et $\mid$ aret;

5 Et quia $\mid$ sic flo $\mid$ ret $^{\mathrm{P}}$ flo $\mid$ rens, sic $\mid$ transit et $\mid$ aret,

Decli|nemus e|am, ${ }^{\mathrm{P}}$ magis $\mid$ inqui|rendo fu|turam.

Iam pre|cessi|sti, ${ }^{\mathrm{P}}$ iam | regula, | Bruno, fu|isti,

Iam con|clusi|sti ${ }^{\mathrm{P}}$ quod | caute | proposu|isti.

Flore|bas mun $\mid \mathbf{d o}^{\mathrm{P}}$ fragi|li, sed $\mid$ flore ca|duco

10Sprevi|sti mun $\mid$ dum $^{\mathrm{P}}$ fragi|lem flo|remque ca|ducum,

Contem|plati|ve ${ }^{\mathrm{P}}$ que|rens suc|cedere | vite,

Quam tibi $\mid$ conce $\mid$ dat, ${ }^{\mathrm{P}}$ qui $\mid$ secla per $\mid$ omnia $\mid$ regat.

[York (cathedral S. Peter)], no 105, 141, p. 334

Item Ricardus ad eundem

1Eter|nam se $\mid \mathrm{dem}^{\mathrm{P}}$ no|bis in|vidit et $\mid$ edem

Eter|ne se $\mid$ dis $^{\mathrm{P}}$ fac|tus prior $\mid$ exul et $\mid$ edis,

Arboris | illici|te ${ }^{\mathrm{P}}$ dum | gustum | suggerit | Eve.

Gustat, | fit gus $\mid \operatorname{tans}^{\mathrm{P}}$ mor|talibus | addita | fatis

5Porre|xitque vi|ro, ${ }^{\mathrm{P}} \mathrm{ca} \mid$ su simi|lis rui|turo.

Immor|talis e|rat, ${ }^{\mathrm{P}}$ si|cut dei|tatis $\mathrm{i} \mid$ mago,

Immor|talis e|rat, ${ }^{\mathrm{P}}$ cos|tis e|ducta vi|rago;

Sed mox $\mid$ post gus $\mid$ tum $^{\mathrm{P}}$ mor|tales $\mid$ effici|untur

Deiec|tuque gra|vi ${ }^{\mathrm{P}}$ para|diso $\mid$ deici|untur.

10Intra|vit mun $\mid$ dum $^{\mathrm{P}}$ mors | effera, | subdidit | illum

Legibus | illa su|is, ${ }^{\mathrm{P}}$ mori|tur, qui | nascitur | omnis.

Parcere | non no|vit, ${ }^{\mathrm{P}}$ sed | nec tibi, | Bruno, pe|percit.

Apposu|it patri|bus, ${ }^{\mathrm{P}}$ iam | sit tibi | vivere | Christus

Et rap|tum mun $\mid$ do $^{\mathrm{P}}$ te $\mid$ restitu|at para|diso.

[York (cathedral S. Peter)], no 105, 142, p. 334-335

Item Wilhelmus ad eundem

1 Carmina $\mid$ si pos $\left|\operatorname{sent}^{\mathrm{P}} \tan \right|$ to $^{\mathrm{H}}$ pro|desse pa|trono, Iam prod $\mid$ esset $\mathrm{e}\left|\mathrm{i}^{\mathrm{D}}\right|$ musa ca|nora me $\mid \mathbf{i}$.

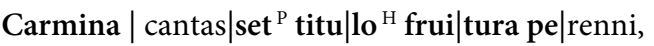
Mille mo|dis $\tan \mid$ to $^{\mathrm{D}} \mid$ psalleret | illa vi|ro, 
5Psalleret | illa vi|ro, ${ }^{\mathrm{P}}$ studi|osa | mente ma|nuque

Linguaque | magnifi|cum ${ }^{\mathrm{D}}$ | magnifi|caret e|um.

Sed quia $\mid$ defunc $\mid$ to $^{\mathrm{P}} \mathrm{ni} \mid$ prosunt $\mid$ carmina | laudis,

Virtus | summa De $\left|\mathbf{i}^{\mathrm{D}}\right|$ propiti|etur $\mathrm{e} \mid \mathbf{i}$,

Torrue|rat Phe|bus ${ }^{\mathrm{P}}$ bis $\mid$ sex oc|toque di|ebus

10Libram, | cum vi $\left|\operatorname{ta}^{\mathrm{D}}\right|$ dis $\mid$ cessit ab | hac ere|mita.

Chester (S. Peter), no 105, 143, p. 335

Ad titulum Sancti Petri Castrensis ecclesie

Gloria | iusto|rum, ${ }^{\mathrm{P}}$ so|latia | sola re|orum

Bruno fu|it vi|vens ${ }^{\mathrm{D}}$ | parque do|lor mori|ens.

Toti|us cle|ri ${ }^{\mathrm{P}}$ decus, | exem|plar quoque | veri,

Mors dolor! | eripu|it, ${ }^{\mathrm{D}}$ | dum pater | occubu|it.

5 Effec|tus mona|chus ${ }^{\mathrm{P}}$ prius, ${ }^{\mathrm{H}} \mid$ hinc ere $\mid$ mitaque $\mid$ rectus

Gratia $\mid$ summa De $\left|\mathbf{i}^{\mathrm{D}}\right|$ propiti|etur e|i.

Huc ten|dunt vo|ta, ${ }^{\mathrm{P}}$ quo | propiti|atio | tota

Sit fragi|li vi|te ${ }^{\mathrm{D}} \mid$ sub brevi|tate si|te.

Huic fuit $\mid$ octo $_{\text {bris }}{ }^{\mathrm{P}}$ im $\mid$ manibus $\mid$ edita $\mid$ probris

10 Finis $\mid$ sexta di|es, ${ }^{\mathrm{D}}$ | sit sibi $\mid$ iam requi|es.

[Chester (S. Peter)], no 105, 144, p. 335

Item alius titulus eiusdem

1 Perso|ne titu|lus ${ }^{\mathrm{P}}$ sub $\mid$ nomine $\mid$ pretitu|latus

Virginis | et Ma|tris, ${ }^{\mathrm{P}}$ quam | post Domi|num colit | orbis,

Diver|sos luc|tus ${ }^{\mathrm{P}}$ mun|dus sonat | undique | totus,

Ad mul|tas mor|tes ${ }^{\mathrm{P}}$ se|cum du|cendo co|hortes.

5 Proster|nit cunc|tos ${ }^{\mathrm{P}}$ sa|cro bap|tismate | lotos,

Sicut $\mid$ demons $\mid$ trat $^{\mathrm{P}}$ Bru|nonis | clara lu|cerna.

Is cum $\mid$ multo|rum ${ }^{\mathrm{P}}$ lau|detur | voce vi|rorum,

Sit meri|tis mo|rum ${ }^{\mathrm{P}}$ spec|tabilis | eximi|orum.

Lege ta $\mid$ men mor $\mid$ tis $^{\mathrm{P}}$ sors $\mid$ hunc tulit | impia | portis.

10Flemus | vobis|cum, ${ }^{\mathrm{P}}$ sed o|pus quid ad | haec nisi | Christum

Poscere, | quod requi|es ${ }^{\mathrm{P}}$ sibi $\mid$ sit post $\mid$ funera | perpes.

Ut ves $\mid$ tris func $\mid$ tis $^{\mathrm{P}}$ dedi|mus, sic $\mid$ vos date $\mid$ nostris. 
Malmesbury (S. Mary, S. Aldhelm), no 105, 145, p. 335-336

T. Sancte Mariae sanctique Aldelmi, episcopi et confessoris Malmesbenensis ecclesie

1 Hic bonus $\mid$ athle $\mid$ ta,${ }^{\mathrm{P}} \mathrm{cu} \mid$ ius cele|berrima | vita

Istic | narra|tur, ${ }^{\mathrm{P}}$ lau|dabilis | esse pro|batur.

Nam si | sic vi|xit, ${ }^{\mathrm{P}}$ pre $\mid$ sens ${ }^{\mathrm{H}}$ ut $\mid$ chartula | dixit,

Et si $\mid$ munifi|cus ${ }^{\mathrm{P}}$ fuit $\mid$ et pius $\mid$ atque pu|dicus,

5 Si sibi|met par|cus ${ }^{\mathrm{P}}$ fuit, | indi|gnis quoque | largus,

Si cal|cator o|pum, ${ }^{\mathrm{P}}$ si $\mid$ spretor $\mid$ delici|arum,

Si ver|bis cul|tus ${ }^{\mathrm{P}}$ fuit $\mid$ et bene $\mid$ morige|ratus,

Est quid o|pus ver|bo, ${ }^{\mathrm{P}}$ quid | dicere | plura la|boro?

Iam nunc | tanto|rum ${ }^{\mathrm{P}}$ ce|lum tenet | arte bo|norum;

10Nam sibi | pro meri|tis ${ }^{\mathrm{P}}$ est | redditus | astriger | axis.

Nunc igi|tur Bru|no ${ }^{\mathrm{P}}$ le|tatur et $\mid$ heret in $\mid$ uno.

Unum | susce $\mid$ pit $^{\mathrm{P}}$ Bru|no, qui | multa re|liquit.

Est tamen | hoc so|lum ${ }^{\mathrm{P}}$ cunc|tis pres|tantibus | unum

Si quam | sit ma|gnum, ${ }^{\mathrm{P}}$ iuvat | hoc ad|discere | lucrum,

15Sat di|cam brevi|ter ${ }^{\mathrm{P}}$ pau|cisque do|cebo pa|tenter.

Susce|pit Chris|tum ${ }^{\mathrm{P}}$ so|lamen | dulce la|borum,

Quid pro|dest igi|tur, ${ }^{\mathrm{P}}$ quod | nos sibi | versifi|camur?

Sed puto, | profice $\mid$ re, ${ }^{\mathrm{P}} \mathrm{si} \mid$ dico 'De|us, mise|rere'.

Ast quoni|am ne $\mid \mathrm{mo}^{\mathrm{P}}$ pec|caminis | est sine | nevo,

20Si quod ha|bet faci|nus, ${ }^{\mathrm{P}}$ tu bone $\mid$ terge De|us.

\section{Rouleau de Mathilde abbesse de la Trinité de Caen, $n^{\circ} 114$}

Normandie

Caen (S. Etienne), no 114, 1, p. 399

\section{T. Sancti Stephani Cadomi}

Que tibi, | Mathil| dis $^{\mathrm{P}}$ lau|dum pre|conia | dentur?

Digna qui|dem ${ }^{\mathrm{T}}$ que|sita di| $\mathrm{u}^{\mathrm{H}}$ non | inveni|entur.

Laus mona|charum, | lux, ${ }^{\mathrm{P}}$ honor $\mid$ et dia|dema fu|isti.

Flent tibi $\mid$ subjec|te, ${ }^{\mathrm{P}}$ quia | morti | succubu|isti,

5Congau|dendo tu|ae ${ }^{\mathrm{P}}$ vi|tae ${ }^{\mathrm{H}}$ de $\mid$ morte que|runtur. 
Veraque $\mid$ de ve $\mid$ ris, ${ }^{\mathrm{P}}$ de $\mid$ multis $\mid$ pauca lo|quuntur.

Cumque tu|as cupi|unt ${ }^{\mathrm{P}}$ boni|tates | amplifi|care

Illas | diminu| unt ${ }^{\mathrm{p}}$ quas | non pos|sunt reci|tare.

Dicunt | pauxi||lum, ${ }^{\mathrm{P}}$ quae | dicunt | vera pro|bantur;

10 Propter | tantil|lum, ${ }^{\mathrm{P}}$ to|tum ${ }^{\mathrm{H}}$ di|xisse pu|tantur.

Prima si|bi ma|ter, ${ }^{\mathrm{P}}$ es $\mid$ vitae | norma se|cundae.

Huic sis $\mid$ exem|plum, ${ }^{\mathrm{P}}$ quia | non opus | est ali|unde.

${ }^{52}$ Fortis ad $\mid$ omnia $^{\mathrm{D} 2} \mid$ gesta vi|rilia ${ }^{\mathrm{D} 4} \mid$ sufficis $\mid$ isti.

Maxima $\mid$ praem $i a^{\mathrm{D} 2} \mid$ per tua ${ }^{\mathrm{D} 3} \mid$ praelia $^{\mathrm{D} 4} \mid$ promeru|isti.

15Mors tibi | gloria ${ }^{\mathrm{D} 2} \mid$ celica $^{\mathrm{D} 3} \mid$ gaud $i a^{\mathrm{D} 4} \mid$ dat tibi $\mid$ funus.

Post obi|tum tribu|iti ${ }^{\mathrm{P}}$ meri|tum ${ }^{\mathrm{H}}$ tibi ${ }^{\mathrm{D}}{ }^{\mathrm{P}} \mid$ trinus et $\mid \underline{\text { unus. }}$

Est nimi $\mid$ um $^{\mathrm{T}}$ te $\mid$ flere ${ }^{\mathrm{F}}$, pi| $\mathrm{um}^{\mathrm{H}}$ te $\mid$ commemo|rari,

Quamvis | non sit o|pus, ${ }^{\mathrm{P}}$ est $\mid$ pro te $\mid$ dulce pre|cari.

O ma|ter dul|cis, ${ }^{\mathrm{P}}$ tibi | sit coe|leste be|amen.

20Hic scrip|tis sit $\mathrm{i} \mid \mathrm{dem}^{\mathrm{P}}{ }^{\mathrm{c}}$ cunc|tisque fi|delibus. | Amen.

S. Gabriel, $\mathrm{n}^{\circ} 114,2$, p. 400

\section{T. Sancti Gabrielis}

1Signat $\mid$ pictu $\mid r a^{\mathrm{P}}$ quia $\mid$ non est $\mid$ mors noci|tura

Cui vult | propiti $\mid a^{\mathrm{P}}$ fie $\mid \mathbf{r i}^{\mathrm{H}}$ pia | virgo Ma|ria.

Dira fu|it mor $\mid$ tis ${ }^{\mathrm{P}}$ lex $\mid$ quondam, | quam leo $\mid$ fortis

In cruce $\mid$ prostra $\mid$ vit ${ }^{\mathrm{P}}$ mori|ens ${ }^{\mathrm{H}}$ mun|dumque pi|avit.

${ }^{53}$ Non erat $\mid$ hostia ${ }^{\mathrm{D} 2} \mid$ quae mori|entia ${ }^{\mathrm{D} 4} \mid$ morte pi| $\underline{\text { aret. }}$

Si foret $\mid$ hosti $a^{\mathrm{D} 2} \mid$ digna per $\mid$ omnia $^{\mathrm{D} 4} \mid$ sacrifi|caret

Hanc homo. | Non erat ${ }^{\mathrm{D} 2} \mid$ hic neque ${ }^{\mathrm{D} 3} \mid$ venerat $^{\mathrm{D4}} \mid$ ut repa|raret

Vel pere|untia, ${ }^{\mathrm{D} 2} \mid$ vel $^{\mathrm{P}}$ mori|entia ${ }^{\mathrm{D} 4} \mid$ vivifi|caret.

Ergo de $\mid$ cens fuit ${ }^{\mathrm{D} 2} \mid$ et $^{\mathrm{P}}$ bene $\mid$ congruit ${ }^{\mathrm{D} 4} \mid$ utili|tati,

10Ut fie|ret Deus ${ }^{\mathrm{D} 2} \mid$ hostia $^{\mathrm{D} 3} \mid$ qua reus ${ }^{\mathrm{D} 4} \mid$ integri|tati

Redditus $^{\mathrm{D} 1} \mid$ est homo. ${ }^{\mathrm{D} 2} \mid$ Cum deus ${ }^{\mathrm{D} 3} \mid$ est homo ${ }^{\mathrm{D} 4}$ | virgine | natus,

Tunc homo | funditus ${ }^{\mathrm{D} 2} \mid$ antea $\mid$ perditus ${ }^{\mathrm{D4}}$ | est reno|vatus.

Fit Deus $\mid$ omnia ${ }^{\mathrm{D} 2} \mid$ presbiter, $\mid$ hostia. ${ }^{\mathrm{D} 4} \mid$ Crux fuit $\mid$ ara.

52. Sur les vers 13-15, appelés tripertiti dactylici, voir Introduction.

53. Les quinze vers suivants sont également des tripertiti dactylici. 
Hac cadit $\mid$ hostia, ${ }^{\mathrm{D} 2} \mid$ mors ruit $\mid$ impia, ${ }^{\mathrm{D} 4} \mid$ pestis a|mara

15In cruce $\mid$ sternitur, ${ }^{\mathrm{D} 2}$ | hac caro $\mid$ pungitur. ${ }^{\mathrm{D} 4} \mid$ Unde la|vamur

Hinc aqua | profluit. ${ }^{\mathrm{D} 2} \mid$ Hinc cruor $\mid$ effluit ${ }^{\mathrm{D} 4} \mid$ unde pi|amur.

Ergo pa|tet via, ${ }^{\mathrm{D} 2} \mid$ celica $\mid$ menia ${ }^{\mathrm{D} 4} \mid$ qua repe|tamus,

Vita fi|delibus ${ }^{\text {D2 }}$ | est Deus $\mid$ omnibus. ${ }^{\text {D4 }}$ | Hunc rogi|tamus

Det veni|am tibi. ${ }^{\mathrm{D} 2} \mid \mathrm{Te}$ soci|et sibi, ${ }^{\mathrm{D} 4} \mid$ fons boni|tatis,

20Christus, | Mathil|dis $;^{\mathrm{P}}$ tibi | detur | vita per|hennis,

Denique | fratribus ${ }^{\mathrm{D} 2}$ | atque so|roribus ${ }^{\mathrm{D} 4}$ | hic titu|latis

Det vi|tae mu| nus $^{\mathrm{P}}$ Deus, | est qui | trinus et | unus. Amen.

Bayeux (cathédrale N.-D), no 114, 5, p. 401-402

T. Sanctae Mariae Baiocensis ecclesiae

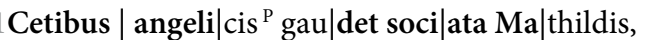

Si sem|per vi|xit, ${ }^{\mathrm{P}}$ no|bis ut | littera | dixit!

Sed qui | munda $\mid$ no $^{\mathrm{P}}$ sor $\mid$ descunt $\mid$ pulvere $\mid$ cuncti

Nodos | pecca|ti ${ }^{\mathrm{P}}$ rum|pat mise|ratio | Christi;

5 Insuper | et requi|em ${ }^{\mathrm{P}}$ tribu|at super | astra per|hennem,

Que preci|bus ius $\mid$ tis ${ }^{\mathrm{P}}$ do|netur $\mid$ subtitu|latis. Amen.

Fontenay-[le-Marmion], S. Etienne, no 114, 7, p. 403

T. Sancti Stephani Fontanetensis

1 Mundi | Salva|tor, ${ }^{\mathrm{P}}$ coe|li ter|raeque Cre|ator,

Qui reg|nat sum $\left|\mathrm{mi}^{\mathrm{D}}\right|$ dives in $\mid$ arce po|li,

Annuat | huic requi|em ${ }^{\mathrm{P}}$ ma|tri vi|tamque pe|rennem,

Et faci|at sanc|tis ${ }^{\mathrm{D}} \mid$ associ|are su|is.

$5 \mathrm{Necnon} \mid$ et nos $\mid \operatorname{tri}^{\mathrm{P}}$ poti|antur | luce pe|renni,

Ipsius | et vul| tum $^{\mathrm{D}}$ | conspici|ant placi|dum.

\section{Angleterre}

Winchester (S. Mary, S. Edburga), no 114, 11, p. 404-405

T. Gloriosae Dei genitricis Marie et sanctae Eadburgae virginis Wintoniensis ecclesie

1 Proh dolor! | Argen|tum, ${ }^{\mathrm{P}}$ ves|tis, nec | gemma, nec | aurum,

Nec pie $\mid$ tas, ${ }^{\mathrm{P}}$ nec $\mid$ nobili|tas, ${ }^{\mathrm{P}}$ nec | larga po|testas,

Morta|li cui|quam ${ }^{\mathrm{P}}$ pos|sunt de|fendere | vitam. 
Nil igi|tur prod|est ${ }^{\mathrm{P}}$ cumu|latio $\mid$ diviti|arum,

$5 \mathrm{Ni}$ mere $\mid$ amur in $\mid$ his $^{\mathrm{P}}$ con|sortia $\mid$ coelico|larum.

Hec bona $\mid$ multa da|bat ${ }^{\mathrm{P}}$ quae | vita be|ata pro|babat,

Cui Deus $\mid$ in ter|ris ${ }^{\mathrm{P}}$ cul|men do|navit ho|noris.

Praesen|tem vi|tam ${ }^{\mathrm{P}}$ li|quit cupi|endo pe|titam

Mathil|dis, sum $\mid \mathrm{mi}^{\mathrm{P}}$ de|poscens $\mid$ premia $\mid$ regni.

10Summi $\mid$ summa De $\left|\mathbf{i}^{\mathrm{D}}\right|$ gloria $\mid$ detur e $\mid \mathbf{i}$.

Item

1 Nos ma|tris lau|dem ${ }^{\mathrm{P}}$ meli|us reti|cendo co|lemus.

Nam con|digna lo|qui ${ }^{\mathrm{P}}$ quia $\mid$ non datur, | inde ta|cemus.

Suffici|at tamen $\mid$ hoc $^{\mathrm{P}}$ quod $\mid$ huic ex $\mid$ corde fa|vemus;

Proditur | et quic| $\mid$ quid $^{\mathrm{P}}$ meri|tis minus $\mid$ esse te|nemus.

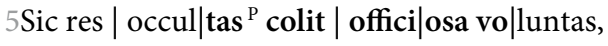

Huic ut $\mid$ majo|rem ${ }^{\mathrm{P}}$ pari|at sine | voce fa|vorem;

Clamet | lingua ta|men: ${ }^{\mathrm{P}}$ 'Tibi | sit, pia | donna, be|amen,

Nosque tu|um mun|das ${ }^{\mathrm{P}}$ faci|at tibi | dogma se|cundas'.

Versus cuiusdam neptis suae

1 Post obi|tum ma|tri ${ }^{\mathrm{P}}$ prope $\mid$ sis, reve|renda $\quad \mathrm{Ma} \mid$ ria

Nomine | Mathil|di ${ }^{\mathrm{P}}$ pia $\mid$ sis, bene|dicta $\quad$ Ma|ria

Hanc bene $\mid$ defen|das, ${ }^{\mathrm{P}}$ for|tis vir|tute $\quad \mathrm{Ma} \mid$ ria

Ne tene $\mid$ bras time $\mid$ at,${ }^{\mathrm{P}}$ te $\mid$ praeduce $\mid$, clara $\quad$ Ma|ria

5Non hos|tis noce|at, ${ }^{\mathrm{p}}$ te $\mid$ visa, | virgo $\quad$ Ma|ria

Insidi|ans tace $\mid$ at, ${ }^{\mathrm{P}}$ te $\mid$ iudice, | domna $\quad \mathrm{Ma}$ |ria

Et frau|des repri|mat, ${ }^{\mathrm{P}}$ te $\mid$ convin|cente, $\quad$ Ma|ria

Mox procul | aufugi|at, ${ }^{\mathrm{P}}$ bona, | te ter|rente, $\quad$ Ma|ria

Hec eat $\mid$ in requi|em, ${ }^{\mathrm{P}}$ te $\mid$ dante, co|lenda, $\quad$ Ma|ria

10Iunga|tur sanc|tis, ${ }^{\mathrm{P}}$ te, | iusta, iu|bente, $\quad$ Ma|ria

Corre|gnetque De $\mid 0,{ }^{\mathrm{p}}$ te $\mid$ presule, $\mid$ digna $\quad \mathrm{Ma} \mid$ ria.

Wilton ('scola'), no 114, 17, p. 406-407

T. Guitoniensis scolae

1 Cur, mors, | sic sae|vis ? ${ }^{\mathrm{P}}$ Cur, | inquam, | sic furi|bunda,

Pluribus $\mid$ exis $\mid$ tis $^{\mathrm{P}}$ satis $\mid$ atque sa|tis treme|bunda?

http://www.unicaen.fr/mrsh/craham/revue/tabularia/print.php?dossier=dossier15\&file=03goullet.xml 
Regia | quam coe $\mid \mathrm{li}^{\mathrm{P}}$ sus $\mid$ cepit $\mid$ gratula|bunda?

Evomis | ecce ma|trem ${ }^{\mathrm{P}}$ tris|tis nimis | ac geme|bunda.

5 Huic prece $\mid$ solva|mus ${ }^{\mathrm{P}}$ quod $\mid$ debe|mus siti|bunda.

Expedit | hoc cunc|tis ${ }^{\mathrm{P}}$ reno|vatis | fluminis | unda.

Shaftesbury (S. Mary, S. Edward), nº 114, 18, p. 407

T. Sancte Mariae et Sancti Edguardi Scephtoniensis ecclesiae

$1 \mathrm{O}$ mors $\mid$ crude $\mid$ lis $^{\mathrm{P}}$ nul|lique pro|bata fi|delis,

O mors | immi|tis, ${ }^{\mathrm{P}}$ ni|gri soror | impia | Ditis,

Quid sic | egis|ti ? $^{\mathrm{P}}$ Quid $\mid$ sic ma|trem rapu|isti?

$\mathrm{O}$ ma|ter di|lecta ${ }^{\mathrm{P}}$ ni|mis, reve|renda ma|gistra,

5Tu, decus | omne tu|is, ${ }^{\mathrm{P}}$ post|quam te | fata tu|lerunt,

Qualiter | ecclesi|ae ${ }^{\mathrm{P}}$ ceci|dit ro|busta co|lumna!

Sed non $\mid$ omni| no $^{\mathrm{P}}$ ruit $\mid$ haec ro|busta co|lumna;

Semper ho|nos ${ }^{\mathrm{T}}$ no|menque tu|um ${ }^{\mathrm{H}}$ lau|desque ma|nebunt.

Christe De|us, ruti $\mid \mathrm{lo}^{\mathrm{P}}$ di|tans il|lam para|diso,

10Mathil|di mise|rere, ${ }^{\mathrm{F}}$ tu|ae mise|rere pu|sillae,

Quae tibi, | dum vi|xit, ${ }^{\mathrm{P}}$ de|vota | mente co|haesit,

Haesit, o|bedi|vit, ${ }^{\mathrm{P}}$ colu|it, di|lexit, a|mavit.

Exeter (Cathedral S. Mary, S. Peter), no 114, 20, p. 408

T. Sanctae Mariae et Sancti apostoli Exoniensis ecclesiae

1 Indicat $\mid$ effec|tus ${ }^{\mathrm{P}}$ non $\mid$ longos $\mid$ temporis | actus.

Actus | esse bre|ves ${ }^{\mathrm{P}}$ mons|trant no|bis mori|entes.

Vita ca|duca bre $\mid$ vis ${ }^{\mathrm{P}}$ nul|li manet | ista per|hennis.

Decipi|tur lon $\mid g^{\mathrm{P}}{ }^{\mathrm{P}}$ qui | querit | tempora | vitae.

5Huius | defunc|tae ${ }^{\mathrm{P}}$ mun|danae | summula | metae

Longam | spem rese $\mid$ cat $^{\mathrm{P}}$ qua | quisque ma|nentia | querat.

Quae sibi | quaesi|vit, ${ }^{\mathrm{P}}$ Chris|to de|vota, Ma|thildis

Nunc frui|tur requi|e, ${ }^{\mathrm{P}}$ capi|endo $\mid$ premia $\mid$ vitae.

Exeter (S. Nicholas), no 114, 23, p. 408

T. Sancti Nicholai Exoniensis ecclesiae

1Diviti|ae re $\mid$ gum $^{\mathrm{P}}$ cinis | est et | gloria | foenum.

Sicut | flos foe $\mid \mathrm{ni},{ }^{\mathrm{P}}$ sic | transit | gloria | mundi.

Flos et | herba ca $\mid$ dunt, ${ }^{\mathrm{P}} \mathrm{sic} \mid$ corpus et $\mid$ ossa pe|ribunt. 
Bath (scolares), no 114, 28, p. 409

Vox scolarium eiusdem urbis

1 Quid furi|tis non|nae ? $^{\mathrm{P}}$ Quid a|matis | carmen i|nane?

Dicite | rem tan $\mid$ dem, ${ }^{\mathrm{P}}$ rem $\mid$ tandem | dicite | plane.

Quid nos $\mid$ buccicre $\mid \mathrm{pa}^{\mathrm{P}}{ }^{\mathrm{p}}$ ser|monum | mole gra|vatis?

Quid teri|tis tem| $\mid$ pus ${ }^{\mathrm{P}}$ ven|tosaque | verba ro|tatis,

5 Insui|tis ver|sus ${ }^{\mathrm{P}}$ et $\mid$ plora|tus pue|riles?

Quid mors, | sera li|cet, ${ }^{\mathrm{P}}$ ve|nas ir|rupit a|niles?

Nonne pu|det, que|so, ${ }^{\mathrm{P}}$ no|bis in|dicere | fletum,

Quod de|fecit a|nus, ${ }^{\mathrm{p}}$ subi|it quod | femina | loetum?

Certe | iure sub|it ${ }^{\mathrm{P}}$ le|talem | femina | sortem:

10Impor|tavit e| $\mid$ nim $^{\mathrm{P}}$ muli|ebris | suasio | mortem.

Addo quod $\mid$ illi $\mid$ us $^{\mathrm{P}}$ fer $\mid$ tis $^{\mathrm{H}}$ super $\mid$ aethera $\mid$ vitam,

Nec dubi|tatis e $\mid \mathbf{a m}^{\mathrm{P}}$ soli|o re|gni redi|mitam.

Hoc igi|tur quan|tum ${ }^{\mathrm{P}}$ care|at rati|one vi|detis,

Quod vos | de domi|na, ${ }^{\mathrm{P}}$ cum | gaudeat | ipsa, do|letis.

15Proptere|a, mone $\mid \mathrm{o},{ }^{\mathrm{P}}$ moe|rori | ponite | metas,

Et mani|bus plau|sus ${ }^{\mathrm{P}}$ et | laudes | edite | laetas.

Sin lacri|mis ple|nas ${ }^{\mathrm{P}}$ huic | indul|getis ha|benas,

Dandum | livo|ri, ${ }^{\mathrm{P}}$ non | vero | credo do|lori.

Nam mala | si mors | est, ${ }^{\mathrm{P}}$ subit | illam | femina | iure;

20Si bona, | non il|lam ${ }^{\mathrm{P}}$ pati|tur bona | femina | dure.

Evesham (S. Mary, S. Egwin), no 114, 32, p. 410

T. Sanctae Mariae et Sancti Ecgwini Eoveshammii coenobii

1 Virgini|tas sum $\mid \mathrm{mae}^{\mathrm{P}}$ geni|tricis | digna Ma|riae

Mathil|di requi|em ${ }^{\mathrm{P}}$ tribu|at, decus | atque le|vamen,

Quo mors $\mid$ nata|lis ${ }^{\mathrm{P}}$ fiat $\mid$ eius $\mid$ perpetu|alis,

Laete|turque po|lo ${ }^{\mathrm{P}}$ Domi|no su|mens bona $\mid$ solo,

5 Ac nihil | adver|sum ${ }^{\mathrm{P}}$ pati|atur, | sed bene | versum

Sit quic|quid volu|it ${ }^{\mathrm{P}}$ bene, | dixit | iure, vel | egit.

Praefuit | in ter|ris ${ }^{\mathrm{P}}$ ani|mabus; | sentiat | astris

Gaudia | quadra|ti $;^{\mathrm{P}}$ faci|at quod | gratia | Christi. 
Pershore (S. Mary), no 114, 33, p. 410

T. Sanctae Mariae Persorensis ecclesiae

1 Christe De|us, rutil| $\mid \mathrm{o}^{\mathrm{P}}$ di|tans ius|tos para|diso,

Mathil| $\mathrm{de}^{\mathrm{T}}$ mise|rere, ${ }^{\mathrm{F}}$ tu| $\mid \mathrm{ae}^{\mathrm{H}}$ mise|rere pu|ellae,

Quae tibi, | dum vi|xit, ${ }^{\mathrm{P}}$ de| $\mid$ vota $\mid$ mente co|haesit,

Haesit, o|bedi|vit, ${ }^{\mathrm{P}}$ colu|it, di|lexit, a|mavit.

Selby (S. German), no 114, 39, p. 411-412

T. Sancti Germani Salisbiensis coenobii

$1 \mathrm{O}$ vos $\mid$ fulgen|tes ${ }^{\mathrm{P}}$ opi|bus, mun|dana se|quentes,

Cum mise|ri si|tis, ${ }^{\mathrm{D}}$ | vana quid | appeti|tis?

Cur non $\mid$ pensa|tis ${ }^{\mathrm{P}}$ quan|tum noce|at quod a|matis?

Quantum | quippe no|cet ${ }^{\mathrm{D}}$ | vita be|ata do|cet ${ }^{54}$

5 Permanet | ecce pa|rum ${ }^{\mathrm{P}}$ pos|sessio | diviti|arum.

Diviti|ae pere $\mid$ unt $^{\mathrm{D}}{ }^{\mathrm{I}}$ dampna ta|men pari|unt.

Has, quae $\mid$ migra|vit, ${ }^{\mathrm{P}}$ nun $\mid$ quam Ma|thildis a|mavit,

Sed bacu|lo fide $\left|\mathbf{i}^{\mathrm{D}}\right|$ rexit o|vile De|i.

Insig|nita bo $\mid$ nis $^{\mathrm{P}}$ et a|matrix $\mid$ religi|onis

10 Haec ma|trona fu|it, ${ }^{\mathrm{D}}$ | vivere $\mid$ dum potu|it.

Si tamen $\mid$ hanc le $\mid$ sit $^{\mathrm{P}}$ quic|quam viti|i, quod in|haesit,

Indul|gendo, pi|us ${ }^{\mathrm{D}} \mid$ abluat $\mid$ ipse De|us.

Et quoni|am ta|lis ${ }^{\mathrm{P}}$ fuit $\mid$ haec ma|ter speci|alis,

Cum Chris $\mid$ to soli $\left|\mathbf{o}^{\mathrm{D}}\right|$ regnet in $\mid$ aethere $\mid \mathbf{o .}$

Norwich (cathedral Holy Trinity), $\mathrm{n}^{\circ}$ 114, 40, p. 412

Versus Othonis iuvenis

1Commen $\mid$ dant ali $\mid i,{ }^{\mathrm{P}}$ com $\mid$ mendant $\mid$ scripta Ma|thildim

Et vi|tae meri|tum ${ }^{\mathrm{D}}$ | iustici|aeque mo|dum.

Scribere $\mid$ disposu $\left|\mathrm{i}^{\mathrm{P}} \tan \right|$ tae prae $\mid$ conia $\mid$ laudis;

Sed mihi | verbo|rum ${ }^{\mathrm{D}}$ | copia | pauca fu|it.

5Primiti|as Domi| $\mid$ no $^{\mathrm{P}}$ dum | libat | virgini|tatis,

Perpetu|a meru|it ${ }^{\mathrm{D}}$ | virgini|tate fru|i.

54. J'ai corrigé doce en docet. 
Virgo di|cata De $\mid$ o, ${ }^{\mathrm{P}}$ re $\mid$ bus sub $\mid$ tracta ca|ducis,

Perce $\mid$ pit meri|to ${ }^{\mathrm{D}} \mid$ praemia $\mid$ digna su $\mid \mathbf{o}$.

In lau|des gemi|tus ${ }^{\mathrm{P}}$ rede|ant, in | gaudia | fletus:

10 Nil di|gnum lacri|mis ${ }^{\mathrm{D}}$ | vita pe|rennis ha|bet.

Transiit $\mid$ ad vi|tam ${ }^{\mathrm{P}}$ fe|lix et | plena di|erum,

Et meru|it spon $\left|s \mathbf{i}^{\mathrm{D}}\right|$ regna vi|dere su|i.

York (S. Mary), no 114, 41, p. 413-415

T. Sanctae Mariae Eboracensis

Versus Benedicti ${ }^{55}$

1Quam vos | defle|tis, ${ }^{\mathrm{P}}$ non $\mid$ nos de|flemus

o|bisse,

Coeles|tem patri|am ${ }^{\mathrm{P}}$ quam $\mid$ nos spe|ramus

ad|isse.

Hinc ves|tram ma|trem ${ }^{\mathrm{P}}$ non | exhor|remus

a|bisse,

Perpetu|am coe $\mid$ nam ${ }^{\mathrm{P}}$ quam | non dubi|tamus

in|isse.

5Dilec|tae ma|tris ${ }^{\mathrm{P}}$ non $\mid$ debet $\mid$ morte

gra|vari

Quisquis e $\mid$ am cre $\mid \operatorname{dit}^{\mathrm{P}}$ iam | iam super | astra

lo|cari.

Carnea | iam mo|les ${ }^{\mathrm{P}}$ nes|cit lacri|mis

repa|rari;

Spiritus | astra pe|tens ${ }^{\mathrm{P}}$ se $\mid$ scit sine | fine

be|ari.

Vivere | si pos|set, ${ }^{\mathrm{P}}$ vo|bis de|beret

a|mari;

10Vivere | non potu|it, ${ }^{\mathrm{P}}$ vo|bis de|bet

tole|rari.

Funere | pro ca $\mid$ rae $^{\mathrm{P}}$ ni prodest $\mid$

collacri|mari,

Dum lacri|mis ves $\mid$ tris $^{\mathrm{P}}$ vi|tae neque|at

revo|cari.

Non vos $\mid$ flere de $\mid$ cet $^{\mathrm{P}}$ si $\mid$ carnis | debita

solvit,

Nam Sal|vator e $\mid \mathrm{am}^{\mathrm{P}}$ pec|cati | lege

re|solvit;

15Sed gau|dere de $\mid \operatorname{cet}^{\mathrm{P}}$ quod | carnis | claustra

re|liquit.

Nam, nisi $\mid$ falla $\mid$ mur, ${ }^{\mathrm{P}}$ coe $\mid$ lestia | regna

pe|tivit.

Littera | nam prae $\mid$ sens $^{\mathrm{P}}$ no|bis mons|trare

vi|detur

Pro boni|tate su| $\mid \mathrm{a}^{\mathrm{P}}$ quod e|i bona $\mid$ vita

pa|retur.

Littera | iam prae $\mid$ sens $^{\mathrm{P}}$ non $\mid$ debet $\mid$ falsa

pu|tari,

20Quae con|testa|tur ${ }^{\mathrm{P}}$ sed | debent

magnifi|cari.

Littera | iam prae $\mid$ sens ${ }^{\mathrm{P}}$ nunc $\mid$ nobis |

signifi|cavit

55. Dans la copie partielle du XII e siècle (Paris, BnF lat. 12652) les vers sont copiés comme ici, avec un grand espace blanc entre l'avant-dernier mot et le dernier.

http://www.unicaen.fr/mrsh/craham/revue/tabularia/print.php?dossier=dossier15\&file=03goullet.xml 
Quod bene | vos re|xit, ${ }^{\mathrm{P}}$ quod | vos dum | vixit

a|mavit ;

Littera | testa|tur ${ }^{\mathrm{P}}$ quod | vobis | profuit |

illa;

Dum tamen $\mid$ enitu|it ${ }^{\mathrm{P}}$ fo $\mid$ mes vir|tutis in |

illa,

25Praefuit | et vo|bis, ${ }^{\mathrm{P}}$ con|testans | littera |

dicit;

Profuit | atque si|bi, ${ }^{\mathrm{P}}$ dum | carnis | lubrica |

vicit.

Haec Satha|nae laque $\mid{ }^{\mathrm{P}}{ }^{\mathrm{P}}$ mul|to co|namine |

stravit,

Dum se | subiec $\mid \operatorname{tas}^{\mathrm{P}}$ vir|tutibus |

accumu|lavit,

Hostis et $\mid$ anti|qui $^{\mathrm{P}}$ sem|per mo|limina |

fregit,

30Et sibi | subiec|tis ${ }^{\mathrm{P}}$ ius levi|tatis

ab|egit,

Coelica | lucra si $\mid \mathrm{bi}^{\mathrm{P}}$ vi|vendo do|cendo

pa|ravit.

Ut capi|atis i|dem, ${ }^{\mathrm{P}}$ ser|vate quod |

aedifi|cavit.

Regula | iustiti|ae ${ }^{\mathrm{P}}$ fuit | eius | semita |

vitae;

Quam sua $\mid$ facta te|gant ${ }^{\mathrm{P}}$ ves|tisque de|cus

poli|mitae;

35Atque do|mus Domi|ni ${ }^{\mathrm{P}}$ di|lexerat | illa

de|corem,

Cui pro | posse su| $\mid{ }^{\mathrm{P}}$ mul|tum do|navit

ho|norem.

Omnibus | illa bo|nis ${ }^{\mathrm{P}}$ vir|tutibus |

Et do|nec vi|xit, ${ }^{\mathrm{P}}$ sem|per tibi, | Christe,

irradi|abat;

Extol|lamus e $\mid \mathbf{a m}^{\mathrm{P}}$ quia | vivens | tot bona |

va|cabat.

fecit,

40Quae, quod | defle|tis, ${ }^{\mathrm{P}}$ pon|dus vio|labile |

iecit.

Mathil|dem ves|tram ${ }^{\mathrm{P}}$ pari|ter nunc|

magnifi|cemus,

De qua | spem cer|tam ${ }^{\mathrm{P}}$ quod | semper | vivat

ha|bemus.

Sed quia $\mid$ nullus ho $\mid \mathrm{mo}^{\mathrm{P}}$ prae|senti | vivat

in | aevo

Qui do|nec vi|vit ${ }^{\mathrm{P}}$ pecca|ti sit sine |

naevo,

45Mathil|di cul|pae ${ }^{\mathrm{P}}$ si | quid car|nalis

ad|haesit,

Deleat | ipse De|us, ${ }^{\mathrm{P}}$ cui | vivens | semper

in|haesit. Amen.

Vestrae | Mathil $\mid$ dis $^{\mathrm{P}}$ suc|currat | virgo

Ma|ria. Amen.

Propiti|etur e $\mid \mathrm{i}^{\mathrm{P}}$ sanc|tissima $\mid$ virgo

Ma|ria. Amen.

Vestram | Mathil|dem ${ }^{\mathrm{P}}$ tue|atur | virgo

$\mathrm{Ma} \mid$ ria. Amen.

50 Et sit e|i clipe $\mid$ us $^{\mathrm{P}}$ pia $\mid$ mater $\mid$ virgo

Ma|ria. Amen.

\section{Versus Ricardi}

$1 \mathrm{O}$ homi|num sine | laude ge|nus, ${ }^{\mathrm{H}}$ quod, | vivere | natum,

Ipsum | se propri $\left|\mathbf{o}^{\mathrm{D}}\right|$ perdidit $\mid$ arbitri $\mid \mathbf{o}$ ! 
Nil sine | laude ma|gis ${ }^{\mathrm{P}}$ quam | quod na|tura de|coris Non in $\mid$ vita su $\left|\mathbf{o}^{\mathrm{D}}\right|$ iure ca|rere tu|lit.

5Factus ho $\mid \mathrm{mo}^{\mathrm{T}}$ sine $\mid$ fraude ${ }^{\mathrm{F}}$ bo|nus, ${ }^{\mathrm{H}}$ qui, | si volu|isset,

Viveret | aeter|num, ${ }^{\mathrm{D}}$ | nec cari|turus e|rat

Imperi|o re|rum. ${ }^{\mathrm{P}}$ Iam | mente su|perbus i|niqua,

Noluit | utili| $\mid$ ter $^{\mathrm{D}}$ | subditus | esse De|o.

Unde, pri|ore bo| no $^{\mathrm{P}}$ vacu|atus, ad $\mid$ ima lo|catur,

10Effici|turque mi| nor $^{\mathrm{D}} \mid$ inferiore su|o.

Omnis $\mid$ quippe De $\mid \mathbf{i}^{\mathrm{P}}$ sequi|tur na|tura fa|vorem,

Natu|rae re|gem ${ }^{\mathrm{D}} \mid$ destitu|ens homi|nem.

Infe|lix igi|tur ${ }^{\mathrm{P}}$ nun|quid sine | fine per|ibit?

Non, in $\mid$ quam, Deus $\mid$ est $^{\mathrm{D}}$ | qui dedit | auxili|um.

15Ille mo|ri veni|ens ${ }^{\mathrm{P}}$ de $\mid$ nostro $\mid$ sumpsit ut $\mid$ esset

Articu|lum mor|tis ${ }^{\mathrm{D}} \mid$ solvere $\mid$ causa De|o.

Tali $\mid$ nempe mo $\mid$ do $^{\mathrm{P}}$ de $\mid$ nostro $\mid$ nostra re|demit,

Ut foret $\mid$ inde sa|lus, ${ }^{\mathrm{D}}$ | venerat unde do|lor.

Lex tamen $\mid$ ob cul|pam, ${ }^{\mathrm{P}}$ cul|pa pere|unte, re|mansit,

20Ut sem|per mane|at ${ }^{\mathrm{D}}$ | unde la|boret ho|mo.

Id rati|one qui|dem ${ }^{\mathrm{P}}$ cons|tat dic|tante pro|batum,

Quo meli|or propri $\left|\mathbf{o}^{\mathrm{D}}\right|$ spes foret $\mid$ ex meri|to.

Haec tibi, | Mathil|dis, ${ }^{\mathrm{P}}$ tan|torum | causa la|borum

Et levis | et dul $\mid$ cis $^{\mathrm{D}}$ | spe meli|ore fu|it.

25 Hac quoque | de cau|sa ${ }^{\mathrm{P}}$ non | rerum | cura co|egit

Quin re|bus mal|les ${ }^{\mathrm{D}} \mid$ praeposu|isse De|um.

Et quia | fecis|ti, ${ }^{\mathrm{P}}$ pro|sit tibi | gratia | Christi,

Qui tibi | virtu|tem ${ }^{\mathrm{D}}$ | contulit | atque fi|dem.

Versus Petri

1Si pos|set lacri|mis ${ }^{\mathrm{P}}$ revo|cari $\mid$ vestra Ma|thildis,

Iam mul|tis lacri $\mid$ mis $^{\mathrm{D}}$ | es| set revo|cata Ma|thildis.

Sed quia $\mid$ non pos|sunt ${ }^{\mathrm{P}}$ lacri|mae revo|care Ma|thildem,

Ergo pre $\mid$ ces stude $\mid$ ant $^{\mathrm{D}}$ | super | astra lo|care Ma|thildem.

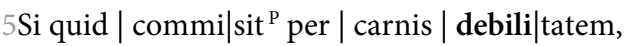

Ignos|cat Chris|tus ${ }^{\mathrm{D}}$ | sibi | per soli|tam pie|tatem. 
Quod peti|tis ves $\mid$ tris $^{\mathrm{P}}$ do|nari $\mid$ praetitu|latis,

Hoc peti|mus nos $\mid$ tris $^{\mathrm{D}} \mid$ do|nari $\mid$ subtitu|latis;

York (cathedral S. Peter), no 114, 42, p. 415-416

T. Sancti Petri Eboracensis ecclesiae

1Heu! res | crude $\mid$ lis, ${ }^{\mathrm{P}}$ res | dirae | conditi|onis!

Pallida $\mid$ mors re|gum ${ }^{\mathrm{P}}$ tur|res ino|pumque ta|bernas

Sorte pa|ri pul|sat, ${ }^{\mathrm{P}}$ nec e|am quis | munere | placat.

Turpes, | formo|sos, ${ }^{\mathrm{P}}$ in|doctos | atque pe|ritos,

5Notos, | igno|tos, ${ }^{\mathrm{P}}$ truti|na com|pensat e|adem.

Quae si $\mid$ diviti $\mid$ is ${ }^{\mathrm{P}}$ pos|set preci|busve mo|veri,

Vel si $\mid$ religi $\mid \mathrm{o}^{\mathrm{P}}$ mol|liret | castaque | vita,

Tu laque $\mid$ os mor|tis ${ }^{\mathrm{P}}$ ru|pisses | iure, Ma|thildis.

Rex tibi | coelo|rum, ${ }^{\mathrm{P}}$ con|sola|tor mise|rorum,

10Pace fru|i ve $\mid \mathrm{ra}^{\mathrm{P}}$ coe|li con|cedat in | aula!

Concio $\mid$ sacra|rum ${ }^{\mathrm{P}}$ te $\mid$ suscipi|at mona|charum!

Lincoln (cathedral S. Mary), no 114, 43, p. 416-417

\section{T. Sanctae Mariae Lincoliensis ecclesiae}

1Heu quan|tum pri|mi ${ }^{\mathrm{P}}$ lacri|marum | culpa pa|rentis

Attulit; | est et ad $\mid$ huc $^{\mathrm{P}}$ ea $\mid$ nostrae $\mid$ sarcina | gentis.

Carmine $\mid$ quo pote $\mid$ ro $^{\mathrm{P}}$ te $\mid$ dignas $\mid$ enume|rare

Laudes, | Mathil|dis, ${ }^{\mathrm{P}}$ vel | pectore | quo memo|rare?

5Debita $\mid$ iam car| $\mid$ nis $^{\mathrm{P}}$ sol|visti $\mid$ morte pu|dica,

Fraude pa|ter pri|mus ${ }^{\mathrm{P}}$ meru|it quae | captus i|niqua.

Caste | vixis|ti; ${ }^{\mathrm{P}}$ vi|tam vi|vens meru|isti.

Morte pi|a mori|ens ${ }^{\mathrm{P}}$ mise|ram mor|tem renu|isti.

Vos de|flere li| $\mid$ quet $^{\mathrm{P}}$; sed | iam gau|dete, so|rores,

10 Hanc ves|tram ma|trem ${ }^{\mathrm{P}}$ mise|ros posu|isse do|lores,

Exiit | exili|um ${ }^{\mathrm{P}}$ pati|enter, | plena di|erum.

In patri|am redi|it ${ }^{\mathrm{P}}$ lux, | gloria, | flos muli|erum.

Coniugis | horta|tum ${ }^{\mathrm{P}}$ si | primus ho|mo renu|isset,

Non sua | posteri| $\mid \operatorname{tas}^{\mathrm{P}}$ mor|talia | iura sub|isset,

15Sed quia | plebs hu|mana ${ }^{\mathrm{F}}$ gra|vi te|lo fuit | icta. 
Pro vete|ri no $\mid \mathrm{xa}^{\mathrm{P}}$ mors | est sibi | poena re|licta.

Haec igi|tur mori|ens, ${ }^{\mathrm{P}}$ quae | versibus | his memo|ratur,

Laetiti $\mid \mathrm{a}^{\mathrm{T}}$ quam | promeru|it ${ }^{\mathrm{H}}$ sine | fine fru|atur.

S. Benet of Hulme, $n^{\circ} 114,46$, p. 418

\section{T. Sancti Benedicti Holmensis ecclesiae}

1 Nil prod|est ani|mae ${ }^{\mathrm{P}}$ met|ri genus $\mid$ omne Ma|thildis;

Sed pro|sunt ani|mae ${ }^{\mathrm{p}}$ pia $\mid$ vota pre|cesque Ma|thildis.

Parcat | huic peti|mus ${ }^{\mathrm{P}}$ Domi|nus pius | ergo Ma|thildi,

Et sanc|tos peti| $\mid$ mus $^{\mathrm{P}}$ suf|fragia | ferre Ma|thildi,

5 Ut pos|sit cu|ram ${ }^{\mathrm{P}}$ Mi|chaelis in|ire Ma|thildis,

Nec sube|at cu|ram ${ }^{\mathrm{P}}$ Sata|nae peri|tura Ma|thildis.

Sique ta|men vi|tae ${ }^{\mathrm{P}}$ sanc|tae fuit | ista Ma|thildis,

Perpetu|ae vi|tae ${ }^{\mathrm{p}}$ copu|latur | iure Ma|thildis.

Ramsey (S. Benedict), no 114, 47, p. 418-419

\section{T. Sancti Benedicti Ramesiae}

1 Si genus $\mid$ et speci|es ${ }^{\mathrm{P}}$ mun|dique po|tentia | leto

Obsta|rent ${ }^{\mathrm{T}}$ pos|sentque di|es ${ }^{\mathrm{H}}$ pro|ducere | nostros,

Multus ho| $\mid \mathrm{mo}^{\mathrm{T}}$ super|esset ad $\mid$ huc ${ }^{\mathrm{H}}$ quem | vita re|liquit,

Forsitan | et Mathil|dis, ${ }^{\mathrm{P}}$ quam iam | pulvis ob|edit.

5 Ergo $\mid$ fortu| $\mid a^{\mathrm{P}}$ cur $\mid$ fiditur $\mid$ in fugi|tiva?

Quisque si $\mid \mathrm{bi}^{\mathrm{T}}$ cito | provide|at: ${ }^{\mathrm{H}}$ mors $\mid$ certa pro|pinquat.

Utque De|us mise|rescat ${ }^{\mathrm{F}} \mathbf{o}$ pemque fe|rat cito $\mid$ nobis,

Dica|mus cunc|ti: ${ }^{\mathrm{P}}$ 'Cle|mens, Deus, | esto Ma|thildi'.

Quod cupi|tis ves $\mid$ tris $^{\mathrm{P}}$ de|functis | poscite | nostris.

S. Albans, $n^{\circ} 114,49$, p. 419-420

T. sancti Albani prothomartyris Anglorum

1 Fletibus $|\mathrm{a} \tan |$ tis $^{\mathrm{P}}$ ca|rae ces|sate so|rores,

Femine|i cor|dis ${ }^{\mathrm{p}} \tan \mid$ dem levi|gando do|lores.

Vixit in | hoc mun|do ${ }^{\mathrm{P}}$ fragi|li vix $\mid$ corpore $\mid$ sospes

Mathil|dis; mun|do ${ }^{\mathrm{P}}$ fuit | haec, sibi $\mid$ mundus et $\mid$ hospes.

5Quae dum | si vi|xit, ${ }^{\mathrm{P}}$ prae|sens ut | cartula | fatur,

In cas|sum fle|tis, ${ }^{\mathrm{P}}$ quoni|am super $\mid$ astra le|vatur.

http://www.unicaen.fr/mrsh/craham/revue/tabularia/print.php?dossier=dossier15\&file=03goullet.xml 
Atque, De|um siti|ens, ${ }^{\mathrm{P}}$ la|bentia $\mid$ despici|ebat;

Actibus | implen $\mid$ do $^{\mathrm{P}}$ ver|bis bona $\mid$ quaeque do|cebat,

Et quia | sustinu| it $^{\mathrm{P}}$ pro $\mid$ Christo $\mid$ quosque la|bores,

10 Nunc sube|at requi|em ${ }^{\mathrm{D}}$ | et sine | fine di|em.

London (Westminster, S. Peter), no 114, 50, p. 420

\section{T. Sancti Patri Westmonasterii}

${ }^{56}$ Vita bre $\mid v i s^{\mathrm{T}}$ satis | esse le $\mid v i{ }^{\mathrm{H}}{ }^{\mathrm{H}}$ sic | iure pro|batur;

Dum mori|tur, ${ }^{\mathrm{T}}$ cui | lux ori|tur $;{ }^{\mathrm{H}}$ sed $\mid$ dum repa|ratur,

In meli|us ${ }^{\mathrm{T}}$ tran|sit pe $\mid i u s,{ }^{\mathrm{H}}$ si | vita pro|batur.

Nam quod $e \mid r a t,{ }^{\mathrm{T}}$ sed $\mid$ mortis $e \mid r a t,{ }^{\mathrm{H}}$ meli|us reno|vatur,

5 Inque $\mathrm{D} e \mid i^{\mathrm{T}}$ vir|tute $\mathbf{m} \boldsymbol{e} \mid \boldsymbol{i}^{\mathrm{H}}$ speci|es ani|matur.

Hinc rese $\mid c e t^{\mathrm{T}}$ cul|pasque ne|cet ${ }^{\mathrm{H}}$ qui|cumque vo|catur

Ad regi|men, ${ }^{\mathrm{T}}$ fac|tus speci|men, ${ }^{\mathrm{H}}$ quod | quisque se|quatur.

Iamque $t i\left|b i,{ }^{\mathrm{T}} \mathrm{Ma}\right|$ thildis, $i \mid b{ }^{\mathrm{H}}$ quo | iustus a|matur

Sit requi|es, ${ }^{\mathrm{T}}$ quo | vera qui|es ${ }^{\mathrm{H}}$ Christus videatur.

10 His vos $\mid$ sola $\mid$ mur, ${ }^{\mathrm{P}}$ nos|trisque pe|tita pre|camur.

\section{Normandie}

Lisieux (cathédrale Saint-Pierre), no 114, 61, p. 422-423

T. Sancti Petri Lexoviensis ecclesiae

1 Hanc di|vinus a $\mid$ mor $^{\mathrm{P}}$ sic $\mid$ labe re|movit ab | omni,

Quod meri|to vi|tae ${ }^{\mathrm{D}}$ | regna su|perna co|lit.

Non igi|tur quis|que ${ }^{\mathrm{P}}$ lacri|mas ef|fundat $\mathrm{i}$ |nanes,

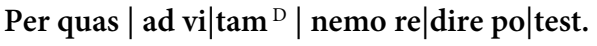

5 Si sibi $\mid$ vult ali $\mid$ quis $^{\mathrm{P}}$ cer|tum con|ferre iu|vamen,

Assidu|as di|cat ${ }^{\mathrm{D}}$ | con pie|tate pre|ces.

Vos qua|rum ma|ter ${ }^{\mathrm{P}}$ dul|cis fuit | illa, so|rores,

Votis $\mid$ et preci|bus ${ }^{\mathrm{D}}$ | ferte fre|quenter o|pem.

Nos quoque | possi|mus ${ }^{\mathrm{P}}$ de|vote $\mid$ mente pre|cari,

10Ut det e|i requi|em ${ }^{\mathrm{D}}$ | cunctipo|tens Domi|nus.

Item

1 Debe|tis ves|tros ${ }^{\mathrm{P}}$ luc|tus ini|bere, so|rores;

56. Ces vers, sauf le dernier, sont appelés trinini salientes. Voir l'introduction. 
Tali | namque mo $\mid \mathrm{do}^{\mathrm{D}}$ | non repa|ratur ho|mo.

Huius ad $\mid$ exem $\mid$ plum $^{\mathrm{P}}$ de $\mid$ betis $\mid$ vivere | cunctae,

Quas Cadu $\mid$ mensis ha $\mid$ bet $^{\mathrm{D}} \mid$ regula, $\mid$ sorde ca|rens.

5Et sic|ut docu $\mid$ it $^{\mathrm{P}}$ vos $\mid$ ire per $\mid$ atria $\mid$ vitae,

Omnes $\mid$ possi|tis ${ }^{\mathrm{D}} \mid$ ire me $\mid$ o moni|tu.

Lisieux (N.-D. [-du Pré]), no 114, 62, p. 423-424

T. Sanctae Mariae Lexoviensis

1 Cuncta pe|tunt lap $\mid$ sum ${ }^{\mathrm{P}}$ quae|cunque vo|cantur ad $\mid$ ortum,

Et rapit $\mid$ in mun $\mid \mathrm{do}^{\mathrm{P}}$ mors $\mid$ omnes $\mid$ ordine | nullo.

Non par|cit iuve $\mid \mathrm{ni}^{\mathrm{P}}{ }^{\mathrm{P}}$ nec $\mid$ scit mise|rerier | ulli;

Sed licet $\mid$ in to $\mid$ to $^{\mathrm{P}}$ domi|netur $\mid$ pessima $\mid$ mundo,

5 Non tamen $\mid$ hanc pati|tur ${ }^{\mathrm{P}}$ Chris|tus, quo | cuncta re|guntur,

In propri|is ser|vis ${ }^{\mathrm{P}}$ quic|quam per|quirere | iuris,

Sed pie|tatis o|pem ${ }^{\mathrm{P}}$ cunc|tis im|pendit e|andem;

Vestrae | qui ma|tri ${ }^{\mathrm{P}}$ con $\mid$ cedat $\mid$ gaudia | coeli.

Ferte iu|vamen $[\mathrm{e} \mid \mathrm{is}]^{\mathrm{P}}$ quos $\mid$ subtitu|lavimus | istic.

Préaux (Saint-Léger), $\mathrm{n}^{\circ}$ 114, 66, p. 425-426

T. Sancti Leodegarii Pratelli in eadem villa

1 Dum sic | polle|ret ${ }^{\mathrm{P}}$ super | hoc, dum | fama vo|laret,

Abstulit | hanc no $\mid$ bis $^{\mathrm{P}}$ gem $\mid$ mam re|gina Ma|thildis,

Tradens | cenobi|um ${ }^{\mathrm{P}}$ sibi | matris | iure re|gendum,

Quod sub ho|nore De $\mid \mathrm{i}^{\mathrm{P}}$ cons $\mid$ truxerat | ipsa Ca|domi.

5 Quae fue|rit post $\mid$ haec, ${ }^{\mathrm{P}}$ non $\mid$ est reci|tare ne|cesse,

Cum titu|lus car|tae ${ }^{\mathrm{P}}$ bene | dixerit. | Et satis | inde

Fundimus $\mid$ ecce pre $\mid$ ces, ${ }^{\mathrm{P}}$ cor|dis sus|piria $\mid$ flentes,

Ut cunc|ti Chris $\mid$ to $^{\mathrm{P}}$ si|gnati $\mid$ crismate | sancto

Subveni|ant ani $\mid$ mae $^{\mathrm{P}}$ ma|tris tam $\mid$ magnifi|candae,

10 Nec minus | illa|rum ${ }^{\mathrm{P}}$ sub|duntur | nomina quarum.

Le Bec[-Hellouin], N.-D., no 114, 68, p. 426-427

T. Sanctae Mariae Becci

1 Dilec|tae ma|tres ${ }^{\mathrm{P}}$ et a|mandae | iure so|rores,

Mors ves|tre ma|tris ${ }^{\mathrm{P}}$ non $\mid$ est plan|genda Ma|thildis.

Ante De|i vul|tum ${ }^{\mathrm{P}}$ mors | est preci|osa bo|norum. 
Illa qui|dem cunc|tis ${ }^{\mathrm{P}}$ fuit $\mid$ exem|plum boni|tatis,

5 Abba|tissa|rum ${ }^{\mathrm{P}}$ laus $\mid$ atque de|cus mona|charum,

Flore pu|dicici|ae ${ }^{\mathrm{P}}$ res|plendens | iustici|aeque;

Cumque De $\mid$ o tene $\mid$ ris $^{\mathrm{P}}$ o $\mid$ blata fu|isset $\mathrm{ab} \mid$ annis,

Fama $\mid$ cuncto|rum ${ }^{\mathrm{P}}$ vir|go per|mansit in | aevum,

Proposi|tum sa|crum ${ }^{\mathrm{P}}$ ser|vans in|contami|natum.

10 Liberet $\mid$ a cunc|tis ${ }^{\mathrm{P}}$ quam | virgo pu|erpura | culpis $^{57}$,

Et soci|et sanc|tis ${ }^{\mathrm{P}}$ in a|maena $\mid$ sede qui|etis.

Tandem | supre $\mid \mathrm{mi}^{\mathrm{P}}$ cum | venerit | hora di|ei,

Iunga|tur posi|tis ${ }^{\mathrm{P}}$ in $\mid$ dextra $\mid$ parte be|atis.

Et vos | pro nos $\mid$ tris $^{\mathrm{P}}$ peti|mus o|rare ve|litis.

S. Wandrille, $\mathrm{n}^{\circ} 114,71$, p. 428

T. sancti Wandregisili abbatis cenobii Fontanellae

1Fons vi|tae dul|cis, ${ }^{\mathrm{P}}$ dis|solvens | pocula | mortis,

Hanc tibi | consoci|a, ${ }^{\mathrm{P}}$ vi|tam requi|emque mi|nistra;

Fratribus $\mid$ et requi| $\mid \mathrm{em}^{\mathrm{P}}$ nos|tris con|cede per|hennem;

Vos ea|dem nos|tris ${ }^{\mathrm{P}}$ preci|bus con|ferre ve|litis.

Montivilliers, N.D., $\mathrm{n}^{\circ}$ 114, 72, p. 430

T. Sanctae Mariae Vilarensis

1Sedibus | aethere|is ${ }^{\mathrm{P}}$ mane|at soci|ata Ma|thildis;

Absit e|i poe $\mid$ na $;{ }^{\mathrm{P}}$ iuvet | illam | virgo Ma|ria.

Angeli|cus coe|tus, ${ }^{\mathrm{P}}$ di|vina | lege re|pletus,

Propiti|us fie $\mid \mathrm{ri}^{\mathrm{P}}$ quae|rat sine | fine Ma|thildi.

5 Ecclesi|ae lu|men ${ }^{\mathrm{P}}$ fuit $\mid$ haec mise|risque iu|vamen.

Coeli $\mid$ doctri|nae ${ }^{\mathrm{P}}$ cupi|ens sub|mittere | sese,

Subdita $\mid$ divi| $\mid$ nis $^{\mathrm{P}}$ re|bus ter|rena re|liquit.

Christus e|i requi|em ${ }^{\mathrm{P}}$ con|cedat ha|bere per|hennem.

S. Victor-[l'Abbaye], no ${ }^{\circ} 14,75$, p. 431

T. sancti Victoris martyris

1Qui fra|terna pe|tunt, ${ }^{\mathrm{P}}$ pen|sent fra|terna re|pendi;

Orent $\mid$ ut tem $\mid$ pus ${ }^{\mathrm{P}}$ veni|at nos|tri mise|rendi.

57. J'ai adopté la correction culpis (au lieu de la $2^{\mathrm{e}}$ occurrence de cunctis), suggérée par Dufour (éd.), 2005-2013, vol. I, p. 427. 
Undique | concuti|mur, ${ }^{\mathrm{P}}$ nul|la spem | dante qui|ete.

Defunc|tis sal| tem ${ }^{\mathrm{P}}$ requi|es sit $\mid$ nescia $\mid$ mete.

Rouen (cathédrale N.-D.), no 114, 76, p. 431-432

T. Sanctae Mariae Rotomagensis

1 Haec sa|crata De $\mid \mathbf{o}^{\mathrm{P}}$ muli|er sum|mi muli|erum

Regis | sponsa| rum $^{\mathrm{D}}$ | mater ho|nesta fu|it.

Haec ma|trona|rum ${ }^{\mathrm{P}}$ ma|trona de|cusque su|arum,

Namque si|bi vi|xit, ${ }^{\mathrm{D}}$ | vixit et | ipsa su|is.

5 Haec tan|dem, meri|tis ${ }^{\mathrm{P}}$ ful|gens et $\mid$ plena di|erum,

Munda|nae sor $\mid$ tis $^{\mathrm{D}} \mid$ conditi|one ru|it.

Ergo | frater|na ${ }^{\mathrm{P}}$ Domi|num pie|tate pre|cemur,

Quatenus $\mid$ aeter| $\mid$ nam $^{\mathrm{D}}$ | donet e|i requi|em.

Item

1Si quit | defunc| to ${ }^{\mathrm{P}}$ ver|sus lacri|maeque va|lerent,

Versus | ac lacri| $\mid$ mas $^{\mathrm{P}}$ ef|fundere | quique stu|derent;

Sed quia $\mid$ nil pro|dest ${ }^{\mathrm{P}}$ ver|sus lacri|masve no|tare,

Sic ete|nim vi| $\mid \operatorname{tam}^{\mathrm{P}}$ nul|lus pote|rit repa|rare,

5 Votis | instan|dum, ${ }^{\mathrm{P}}$ preci|bus reor | invigi|landum,

Ut quic|quid macu|lae ${ }^{\mathrm{P}}$ con|traxit | conditi|one

Corpore $|\mathrm{a} \mathrm{cu}|$ ius $^{\mathrm{P}}$ haec $\mid$ cartula $\mid$ denotat $\mid$ actus,

Donet $\mid$ Christus e $\mid \mathrm{i}^{\mathrm{P}}$ se $\mid$ dem praes $\mid$ tans requi|ei,

Ut duce | virgine $\mid$ is ${ }^{\mathrm{P}}$ Chris|to con|iuncta co|reis,

10 Perpetu|$\left|\mathrm{ae}^{\mathrm{T}} \mathrm{lu}\right| \mathrm{cem}^{\mathrm{P}}$ patri|ae ${ }^{\mathrm{H}}$ mere|atur ha|bere.

Rouen (Saint-Ouen), $\mathrm{n}^{\circ}$ 114, 78, p. 433

T. Sancti Petri Sanctique Audoeni Rothomagensis ecclesiae

1 Haec ma|ter, mo|rum ${ }^{\mathrm{P}}$ probi|tas nu|trixque so|rorum,

Contemp|sit flo|rem ${ }^{\mathrm{P}}$ mun|di, ser|vando pu|dorem.

Ergo, | Messi|a, ${ }^{\mathrm{P}}$ genu|it quem | virgo Ma|ria,

Ne repro|bi tes $\mid$ tes ${ }^{\mathrm{P}}$ noce $\mid$ ant sibi, | gaudia $\mid$ praestes.

Rouen (Sainte-Trinité[-du-Mont]), no 114, 79, p. 433

T. Sanctae Trinitatis Rotomagi

Christe, De|i pa|tris ${ }^{\mathrm{P}}$ vir|tus et $\mid$ gloria $\mid$ matris,

Nostros | erro|res ${ }^{\mathrm{P}}$ mise|ratus | necne do|lores,

http://www.unicaen.fr/mrsh/craham/revue/tabularia/print.php?dossier=dossier15\&file=03goullet.xml 
Qui bona $\mid$ das gra|tis, ${ }^{\mathrm{P}}$ huic $\mid$ tende ma|num pie|tatis,

Ut tibi $\mid$ coniunc $\mid$ ta $^{\mathrm{P}}$ fragi $\mid$ li sit $\mid$ corpore $\mid$ functa,

5 Ac te|cum vi|vat, ${ }^{\mathrm{P}}$ quam | vita | mors fera $\mid$ privat.

Rouen (Saint-Paul), no ${ }^{\circ}$, 80, p. 433-434

\section{T. Sancti Pauli Rothomagi}

1Non hanc $\mid$ condemp $\mid$ net $^{\mathrm{P}}$ sen|tentia | iudici|alis,

Regna su|perna De $\mid i^{\mathrm{P}}$ sibi $\mid$ gratia $\mid$ det veni|alis,

Nec quod | pecca|vit ${ }^{\mathrm{P}}$ per|pes vin|dicta se|quatur,

Qua gau|det requi| $\mid \mathrm{e}^{\mathrm{P}}$ ius|torum | turma fru|atur.

\section{Conches[-en-Ouche], S. Pierre, $n^{\circ}$ 114, 84}

\section{T. Sancti Petri Castellionis}

1 Lux fugi|ens ${ }^{\mathrm{T}}$ et $\mid$ nox veni|ens ${ }^{\mathrm{H}}$ car|men brevi|abit,

Versicu|los pau $\left|\cos ^{\mathrm{D}}\right|$ in rotu|loque da|bit.

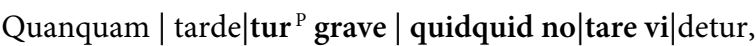

Hoc breve $\mid$ scribo ta|men: ${ }^{\mathrm{D}} \mid$ haec requi|escat. A|men.

\section{S. Sever[-Calvados], no 114, 89, p. 437}

T. Sanctae Mariae sanctique Severi confessoris

1 Felix | semper e| rit $^{\mathrm{P}}$ haec $\mid$ abba|tissa Ma|thildis,

Si bene | sic vi|xit, ${ }^{\mathrm{P}}$ ut | praesens | cartula | dixit;

Terrea $\mid$ posse $\mid$ dit, ${ }^{\mathrm{P}}$ coe $\mid$ lestia $\mid$ semper a|mavit;

Fletibus | ecce ca|ret, ${ }^{\mathrm{D}}$ | gaudia | semper ha|bet;

5 Perpetu|am requi|em ${ }^{\mathrm{D}} \mid$ det sibi $\mid$ Christus. $\mathrm{A} \mid$ men.

Cerisy[-la Forêt], S. Vigor, no 114, 90, p. 437

\section{T. Sancti Vigoris Ceraniensis}

Quam cupi|mus no|bis, ${ }^{\mathrm{P}}$ sint | vobis | dona sa|lutis

Qui fi|nem ma|tris ${ }^{\mathrm{p}}$ trac|tatis | questibus | atris,

$\mathrm{Ob}$ ma|tris lae $\mid$ tum,${ }^{\mathrm{P}}$ mone $\mid \mathrm{o}$ vos, | linquite | fletum:

Tollitur $\mid$ angeli $\mid$ cis $^{\mathrm{P}}$ qui $\mid$ vixit $\mid$ taliter $\mid$ ulnis

5In coe $\mid$ lum, ve $\mid \operatorname{rax}^{\mathrm{P}}$ ut $\mid$ signat $\mid$ epistola | vestra.

Nos tamen | hunc supe $\mid$ ras $^{\mathrm{P}}$ preci|bus tol|lemus in | auras;

Vos ea|dem nos|tris ${ }^{\mathrm{P}}$ per|solvite | iura vi|cissim. 


\section{Lessay, S. Trinité, $n^{\circ}$ 114, 92, p. 438}

\section{T. Sanctae Trinitatis Exaquii}

1 Vos faci|at lae $\mid \operatorname{tas}^{\mathrm{P}}$ qui $\mid$ fecit in $\mid$ aethere $\mid$ metas,

Ut faci|em Re|gis ${ }^{\mathrm{P}}$ cer|natis $\mid$ cunctipo|tentis.

Sit qui | dicat a|men, ${ }^{\mathrm{P}}$ cui $\mid$ sit sub | pectore | flamen.

Pellifer | adveni|ens, ${ }^{\mathrm{P}}$ par|tes nos|tras quoque | tangens,

5 Vester $\mid$ conspicu $\mid \mathrm{am}^{\mathrm{P}}$ de $\mid$ volvit in $\mid$ ordine $\mid$ cartam,

Quae fa|tum ves $\mid$ trae $^{\mathrm{P}}$ ma|tris vi|tam quoque $\mid$ plene

Continet, $\mid$ ad se $\mid$ dem $^{\mathrm{P}}$ quam $\mid$ rex vehat $\mid$ altus et $\mid$ aedem,

Quo gau|dent sanc|ti ${ }^{\mathrm{P}}$ splen|dentes | lumine | coeli,

In requi|e sum $\mid$ ma $^{\mathrm{P}}$ poti|entes $\mid$ pace di|urna.

10Gloria | quae capi $\mid \mathrm{at}^{\mathrm{P}}{ }^{\mathrm{v}}$ vos $\mid$ et vos $\mid$ hoc Deus $\mid$ addat,

Et vos $\mid$ cum ves $\mid$ tra $^{\mathrm{P}}$ vi|vatis $\mid$ matre be|ata,

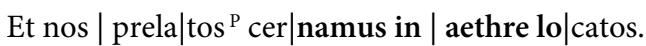

Reddite | vos nos $\mid$ tris $^{\mathrm{P}}$ quod | vultis $\mid$ nos dare | vestris.

\section{Coutances (cathédrale N.-D.), $\mathrm{n}^{\circ}$ 114, 94, p. 439-440}

T. Sanctae mariae Constantiensis eccleasiae

1 Mathil|dis! si | te ${ }^{\mathrm{P}}$ potu|issent | reddere | vitae

Plangere, | trista|ri, ${ }^{\mathrm{P}}$ lacri|mas dare |, versifi|cari,

Fletibus | insta|rem, ${ }^{\mathrm{P}}$ vel | versibus | invigi|larem.

Sed labor $\mid$ hic qua|re, ${ }^{\mathrm{P}}$ si $\mid$ te neque|am repa|rare?

5 Non igi|tur lacri|mis ${ }^{\mathrm{P}}$ aut | versibus | invigi|labo,

Sed preci|bus, psal|mis, ${ }^{\mathrm{p}}$ te | supplice | voce iu|vabo.

Missae | canten|tur, ${ }^{\mathrm{P}}$ cor|dis sus|piria | dentur;

Talia $\mid$ sola| men $^{\mathrm{P}}$ faci|ent his $\mid$ atque iu|vamen.

Linquite | iam planc|tum $;{ }^{\mathrm{P}}$ pro | planctu | reddite | cantum.

10Non est $\mid$ hoc lae |tum ${ }^{\mathrm{P}}$ quo $\mid$ vos dece|at dare | fletum;

De quo | gauden|dum ${ }^{\mathrm{P}}$ cre|do, non | esse do|lendum.

Nam mun|di flo|rem ${ }^{\mathrm{P}}$ sper|nens haec | atque de|corem,

Se cas $\mid$ tigan $\mid$ do, ${ }^{\mathrm{P}}$ car|nem quoque | mortifi|cando,

In se | planta|vit ${ }^{\mathrm{P}}$ vir|tutes, $\mid$ noxia $\mid$ cavit,

15Mundo | pugna|vit, ${ }^{\mathrm{P}}$ Domi|no sua | seque di|cavit. 
Cui se $\mid$ coniun $\mid$ xit $^{\mathrm{P}}$ dum $\mid$ mundi $\mid$ carcere $\mid$ vixit,

Cui se | dona|vit, ${ }^{\mathrm{P}}$ quem | solum | semper a|mavit,

Huic con|iunga|tur ${ }^{\mathrm{P}}$ et a|maena | luce fru|atur.

Avranches, cathédrale (S. André), no 114, 95, p. 440-441

\section{T. Abricensis ecclesiae Sancti Andreae}

1Si qui | scripse|runt ${ }^{\mathrm{P}} \mathrm{Ma}$ |thildis | facta, fu|erunt

Vel sunt $\mid$ veridi|ci, ${ }^{\mathrm{P}}$ nec $\mid$ more lo $\mid$ quuntur a $\mid$ mici.

Depo|nat fle|tus ${ }^{\mathrm{P}} \mathrm{o} \mid \mathrm{lim}$ iunc|tus sibi $\mid$ coetus,

Atque va|cet cu|ra, ${ }^{\mathrm{P}}$ quia $\mid$ non est $\mid$ iam mori|tura.

5Nunc est | gauden|dum, ${ }^{\mathrm{P}}$ vel | votis | subveni|endum,

Pulvere | cum vi|tae ${ }^{\mathrm{P}}$ sit $\mid$ nulla ca|ro sine | lite,

In se | regna|re ${ }^{\mathrm{P}}$ quam | Paulus | flebat a|mare;

Sed dum $\mid$ suppo| $\mid$ ni $^{\mathrm{P}}$ pote|rat mor|tis diti|oni,

[Semper] e|rat flen|dum ${ }^{\mathrm{P}}$ poti|us sem|perque ti|mendum

$10<\ldots \ldots \ldots . . \times$ ve $\mid \mathrm{ri}^{\mathrm{P}}$ ne $\mid$ posset $\mathbf{a} \mid$ more mo|veri

$<\ldots . . .>-$ tu|ra ${ }^{\mathrm{P}}$ post $\mid$ mortem $\mid$ fando fu|tura

$<\ldots \ldots .>$ preci|osa do|lenda ma|lorum

$<\ldots>-$-ire $^{\mathrm{P}}$ nos $\mid$ stringunt $\mid$ vincula $\mid$ iure

$<\ldots \ldots . .>$ ut $\mid$ nulla co|erceat $\mid$ aetas

$15<\ldots \ldots \ldots .>$ si $\mid$ quid dis $\mid$ cedit a|menus.

Sed $\langle\ldots \ldots \ldots\rangle \mid$. dona pre $\mid$ cesque de $\mid$ disse

Qui facit $\mid<\ldots \ldots \ldots . .>$ suc $\mid$ currit a $\mid$ mico.

Item

1 Prima pa|rens ${ }^{\mathrm{T}}$ pri|musque pa|ter, ${ }^{\mathrm{H}}$ dum $\mid$ magnifi|cari

Se cupe|rent, ${ }^{\mathrm{T}}$ subi|to ${ }^{\mathrm{P}}$ lap|su ${ }^{\mathrm{H}}$ se|dem requi|ei

Ami|sere, ${ }^{\mathrm{F}}$ da|tumque fu| it $^{\mathrm{H}}$ sub | morte pe|renni

Triste pi|are ne $\mid$ fas $^{\mathrm{P}}$ et $\mid$ iustas $\mid$ solvere $\mid$ poenas.

5 At Ver|bum ${ }^{\mathrm{T}}$ de $\mid$ patre De $\left|\mathrm{o},{ }^{\mathrm{H}} \mathrm{de}\right|$ lumine | lumen,

Virginis | ex ute $\mid$ ro $^{\mathrm{P}}$ Deus $\mid$ est $^{\mathrm{H}}$ status | inteme|ratae,

Qui cum $\mid[\mathrm{m}]$ ore re $\mid \mathrm{i}^{\mathrm{P}}$ mor $\mid \mathrm{ti}{ }^{\mathrm{H}}$ suc|cumberet, $\mid$ inde

Tanquam | de som $\mid$ no ${ }^{\mathrm{P}}$ sur|gens, ${ }^{\mathrm{H}}$ ut $\mid$ dixerat $\mid$ ante,

Diluit | omne ma|lum, ${ }^{\mathrm{P}}$ pec|cataque | nostra re|solvit, 
10Et dedit | ad patri|am, ${ }^{\mathrm{P}}$ iam | longo | tempore | clauam,

Ut rede|ant homi|nes, ${ }^{\mathrm{P}}$ man|data De|i faci|entes.

At mun|di vi|tam ${ }^{\mathrm{P}}$ fragi|lem ${ }^{\mathrm{H}}$ trans|gressa Ma|thildis

Sancto|rum ${ }^{\mathrm{T}}$ fe|licibus | associ|ata cho|reis,

Obtine $\mid$ at pa $\mid$ cem $^{\mathrm{P}}$ per $\mid$ secula $\mid$ cuncta per|hennem.

Le Mont-Saint-Michel, no 114, 96, p. 441-442

T. sancti Michaelis archangeli de periculo maris

1 Isti|us ma|tris ${ }^{\mathrm{P}}$ fla|men soci|etur in $\mid$ astris

Civibus | aethere|is, ${ }^{\mathrm{P}}$ preci|bus sanc|ti Micha|elis.

Nec mor|tis no|men ${ }^{\mathrm{P}}$ nec a|verni $\mid$ sentiat $\mid$ omen.

Consoci|entur i $\mid \mathrm{bi}^{\mathrm{P}}$ fra|tres hic $\mid$ subtitu|lati.

\section{Rouleau de Vital de Savigny, no 122}

\section{Normandie}

Caen (Saint-Étienne), no 122, 2, p. 524

\section{T. Sancti Stephani Cadomi}

1 Cum sint $\mid$ nota sa|tis ${ }^{\mathrm{P}}$ pre $\mid$ conia $\mid$ strenui|tatis,

Cur a $\mid \operatorname{tantil||lis}{ }^{\mathrm{P}}$ scri|ptis in|sistitur | illis?

Est poti|us flen $\mid$ dum $^{\mathrm{P}}$ cum | flentibus | atque do|lendum,

Quod mors | absque mo|ra ${ }^{\mathrm{P}}$ sic | consu|mat meli|ora

5Dicitur | a vi|ta ${ }^{\mathrm{P}}$ Vi|talis, | sit sibi | vita.

Quotque so|nat no $\mid$ men $^{\mathrm{P}}$ sibi $\mid$ felix $\mid$ compleat $\mid$ omen.

Caen (La Trinité), n⿳0 122, 3

\section{T. Sancti Trinitatis Cadomi}

1 Dum vi|xit, mun $\mid$ do $^{\mathrm{P}}$ quem $\mid$ sprevit $\mid$ cessit ab $\mid$ isto

Vita|lis, sum $\mid$ mus $^{\mathrm{D}} \mid$ sit sibi $\mid$ vita De|us.

Quis vale $\mid$ at fa $\mid \mathrm{ri}^{\mathrm{P}}$ di|gne, vel $\mid$ quis medi|tari

Quam dul $\mid$ ci studi $\left|\mathbf{o}^{\mathrm{D}}\right|$ profuit $\mid$ hic popu|lo?

5Illius | occur|sus ${ }^{\mathrm{P}}$ om|nis metu|ebat in|iquus,

Qui faci|endo ma|lum ${ }^{\mathrm{D}}$ | non timu|ere De|um.

Ille pi|us dic $\mid$ tis $^{\mathrm{P}}$ hos $\mid$ affa|batur a|micis, 
Et, pro|mendo bo|num, ${ }^{\mathrm{D}}$ | linqueret | omne ma|lum

Suade|bat; mul|ti ${ }^{\mathrm{P}}$ sua|dente fu|ere re|tracti

10 Hoc er|rore su $\left|\mathbf{o},{ }^{D}\right|$ seque de $\mid$ dere De $\mid \mathbf{o}$

Huius e|rant comi| tes ${ }^{\mathrm{P}}$ pie|tas, con|cordia ; lites

Vita|bat; sum $\mid$ mus, ${ }^{\mathrm{D}}$ | quo medi|ante, De|us,

Cordibus $\mid$ in mult $\mid$ is ${ }^{\mathrm{P}}$ fun $\mid$ debat $\mid$ semina $\mid$ pacis,

Exemp|tum ta|lem ${ }^{\mathrm{D}} \mid$ morte do|lete pa|trem,

15Fratres, | quox tra| it $^{\mathrm{P}}$ viti|is se|cumque gre|gavit,

Et bona, | dum licu|it, ${ }^{\mathrm{D}}$ | quis docu|menta de|dit.

Iam geme, $\mid$ Norman $\mid \mathrm{na}^{\mathrm{P}}$ tan|to patre | gens vidu|ata:

Non est $\mid$ quo ver $\mid$ bis $^{\mathrm{D}} \mid$ nunc sati|ere bo|nis;

Hic te, | dum licu|it, ${ }^{\mathrm{P}}$ Domi|ni ser|mone ci|bavit.

20 Hoc ali|isque bo|nis ${ }^{D}$ | gaudeat | ipse po|lis. Amen.

Bayeux (Cathédrale N-D.), no 122, 4, p. 525-526

Titulus Sancte Marie Bajocensis ecclesie

1Postquam | Vita|lis ${ }^{\mathrm{P}}$ ceci|dit, rigor $\mid$ ecclesi|alis,

Deso|lato|rum ${ }^{\mathrm{P}}$ so|latio, | dux mona|chorum,

Vobis | esse da|tum, ${ }^{\mathrm{P}}$ fra|tres, fle|tis grave | fatum.

Quare | posse mo|ri ${ }^{\mathrm{P}}$ per|mittitur | illius | ori,

5Quod di|vino|rum ${ }^{\mathrm{P}}$ fons | extitit | eloqui|orum?

Huius in $\mid$ ingres $\mid \mathrm{su}^{\mathrm{P}}$ con $\mid$ cordia $\mid$ paxque re|cessu.

Quo se | verte|bat, ${ }^{\mathrm{P}}$ ibi | semper | profici|ebat.

Nulli $\mid$ nota sa|tis ${ }^{P}$ est $\mid$ summa su|e boni|tatis.

Ille re|quisi|tus ${ }^{\mathrm{P}}$ con|missa re|ferre, pe|ritus

10 Protulit $\mid$ exten $\mid$ ta $^{\mathrm{P}}$ que | sumpserat | ante ta|lenta.

Nunc tribu|atur e $\mid \mathrm{i}^{\mathrm{P}}$ locus $\mid$ eter|ne requi|ei.

(Orate pro nostris, liste de défunts de la cathédrale)

Pacis o|pus ${ }^{\mathrm{T}}$ Vi|talis e|ras. ${ }^{\mathrm{H}}$ Nunc $\mid$ clausus in | amtro,

Dampna, do|lor, patri|e, ${ }^{\mathrm{D}}$ | fratribus | ipse ia|ces.

Si tibi $\mid$ mors preti $\mid \mathrm{o}^{\mathrm{P}}$ iam | vellet | par esse, | pro te

15 Hermi | danda fo $\mid$ ret $^{\mathrm{D}} \mid$ dives a|rena si|bi.

Sed mors $\mid$ diviti|as ${ }^{\mathrm{P}}$ non $\mid$ unquam $\mid$ curat ha|bere, 
Equaque | diviti|bus ${ }^{\mathrm{D}}$ | pauperi|busque ue|nit.

Mors de | te rapu|it ${ }^{\mathrm{P}}$ in | te quod $\mid$ iuris ha|bebat.

Spiritus | ante De|um ${ }^{\mathrm{D}}$ | quod caro | fecit ha|bet.

20Flent ceci|disse du|cem ${ }^{\mathrm{P}}$ fra|trem, Nor|mannia | lucem;

Sed pro|desse pa|rum ${ }^{\mathrm{P}}$ reor $\mid$ effu|sus lacri|marum;

Hi flent | abba|tem ${ }^{\mathrm{P}}$ subi|isse ne|cis graui|tatem,

Spiritus | abba|tis, ${ }^{\mathrm{P}}$ nichil $\mid$ hinc habet | utili|tatis.

Mors est | Vita|lis ${ }^{\mathrm{P}}$ dis|sessio | spiritu|alis.

25Non est | creden|dum ${ }^{\mathrm{P}}$ quod | morti | ius sit [ha|bendum]

Ullum | per fa|tum ${ }^{\mathrm{P}}$ sibi | surripu|isse be|atum.

Credo fo|res ce|li ${ }^{\mathrm{P}}$ rese|ratas $\mid$ cuique fi|deli.

Que tulit, $\mid$ abba| $\mid$ ti $^{\mathrm{P}}$ sint $\mid$ omnia $\mid$ commodi|tati.

Lisieux (cathédrale Saint-Pierre), no ${ }^{\circ 22}$, 7, p. 527

\section{T. Sancti Petri Lexoviensis}

1 In requi|e de|git, ${ }^{\mathrm{P}}$ si | crimina | queque sub|egit;

Si minus |, in pe|nis, ${ }^{\mathrm{P}}$ ex|utus | carnis ha|benis;

Vel reci|pit pe|nam, ${ }^{\mathrm{P}}$ vel $\mid$ de cer|tamine | palmam.

Hic bene $\mid$ certa|vit, ${ }^{\mathrm{P}}$ Domi|num sapi|enter a|mavit;

5Et quem $\mid$ dile $\mid$ xit $^{\mathrm{P}}$ Deus $\mid$ hunc super $\mid$ ethera $\mid$ vexit.

Angeli|cus ce|tus ${ }^{\mathrm{P}}$ de $\mid$ tanto $\mid$ compare | letus,

Hunc col|leta|tur ${ }^{\mathrm{P}}$ et $\mid$ collau|dans vene|ratur.

Ergo pi|is preci|bus ${ }^{\mathrm{P}}$ pa|trem super $\mid$ astra lo|catum,

Hunc ex|ore|mus ${ }^{\mathrm{p}}$ veni|amque lo|cumque pa|ratum.

Grestain (N.-D.), no 122, 12, p. 530-531

\section{T. Sancte Marie Gresteni}

${ }^{158}$ Carta bre|vis ${ }^{\mathrm{T}}$ sen|susque le|vis ${ }^{\mathrm{H}}$ car|men brevi|avit

Nam brevis $\mid$ est, ${ }^{\mathrm{T}}$ et $\mid$ mens levis $\mid$ est,${ }^{\mathrm{H}}$ nec $\mid$ multa pro|bavit.

Per vari|as ${ }^{\mathrm{T}}$ mul|tasque vi|as ${ }^{\mathrm{H}}$ est $\mid$ carta fe|renda.

Proptere $\mid \mathrm{a}^{\mathrm{T}}$ iam $\mid$ non in $\mathrm{e} \mid \mathrm{a}^{\mathrm{H}}$ sunt $\mid$ multa lo $\mid$ quenda,

58. Les vers 1-8 sont des trinini salientes: les rimes internes se trouvaient généralement après les coupes trihémimère et hepthémimère (NORBERG, 1958, p. 66-67). 
5 Hinc igi|tur, ${ }^{\mathrm{T}}$ quia $\mid$ sic peti|tur, ${ }^{\mathrm{H}}$ iam $\mid$ provide|atur

Utili|us ${ }^{\mathrm{T}}$ quic|quam ${ }^{\mathrm{P}}$ meli|us ${ }^{\mathrm{H}}$ que $^{59}$ quod | adici|atur.

Ecclesi $\mid \mathrm{e}^{\mathrm{T}}$ vox $\mid$ sancta pi|e, ${ }^{\mathrm{H}}$ quam $\mid$ grex petit $\mid$ iste

Flendo, pi|um ${ }^{\mathrm{T}}$ ferat $\mid$ auxili|um ${ }^{\mathrm{H}}$ per $\mid$ te, bone, | Christe,

Patri | Vita|li. ${ }^{\mathrm{P}}$ Dig|nus sit $\mid$ nomine | tali,

10Vivat | Vita|lis, ${ }^{\mathrm{P}}$ sit e $\mid \mathrm{i}$ lux | perpetu|alis.

Vivat in $\mid$ eter|num, ${ }^{\mathrm{P}}$ det e| $\mid \mathrm{i}$ dia|dema su|pernum,

Cui se $\mid$ dona|vit, ${ }^{\mathrm{P}}$ Chris|tus, quem | semper a|mavit,

Cui se | devo|vit, ${ }^{\mathrm{P}}$ quem | non a | corde re|movit.

Lector et $\mid$ audi|tor, ${ }^{\mathrm{P}}$ nos|trum rele|gendo pre|camen,

15De patris | interi|tu, ${ }^{\mathrm{P}}$ mes|tus res|pondeat:|Amen.

Item

1 Non stupe $\mid$ as homi $\mid$ nem ${ }^{\mathrm{P}}$ per $\mid$ casum | mortis i| nanem

Ex se $\mid$ materi $\mid \mathrm{em}^{\mathrm{P}}$ dare $\mid$ paulo | post lutu|lentam,

Quam, velu|ti fer|tur, ${ }^{\mathrm{P}}$ ver|mes de $\mid$ tabe cre|ati

Diminu|unt peni|tus, ${ }^{\mathrm{P}}$ quos | omnes | denique | tollit

5Esuri|es es $\mid$ ce, ${ }^{\mathrm{P}}$ iam $\mid$ prorsus $\mid$ defici|ente

Quem come|dant vic|tu, ${ }^{\mathrm{P}}$ prop|ter quod | sic mori|untur.

Unus|quisque qui|dem ${ }^{\mathrm{P}}$ protho|plasti $\mid$ crimine $\mid$ sortem

Suscipi|et lap|sus, ${ }^{\mathrm{P}}$ quo | vite | deseret | usus.

Istud | terrige $\mid$ ne $^{\mathrm{P}}$ Deus $\mid$ haud in $\mid$ notuit $\mid$ Ade:

10Limus es, | exeru|it, ${ }^{\mathrm{P}}$ na|ture | iura sub|ibis,

Pulveris | in gle $\mid$ bam $^{\mathrm{P}}$ redi|geris | praescius | Adam.

O genus | huma|num, ${ }^{\mathrm{P}}$ per|labile, | tam mise|randum,

Cor tene $\mid$ as mes $\mid$ tum $^{\mathrm{P}}$ cum $\mid$ presto|leris a|marum.

Ah tibi | ventu|rum, ${ }^{\mathrm{P}}$ iam | non dubi|e tole|randum.

15Luctus, | vade do|mum. ${ }^{\mathrm{P}}$ Lu|sorum | desere | luxum.

Non est | auxili|um ${ }^{\mathrm{P}}$ quo | decli|nare fu|turum

A te $\mid$ praevale $\mid$ as, ${ }^{\mathrm{P}}$ apud $\mid$ omnes $\mid$ cum memo|rata

Extite $\mid$ rint, ${ }^{\mathrm{T}}$ hec $\mid$ et fore $\mid$ natu|ralia $\mid$ nemo

59. Comme l'a montré Dag Norberg (Ibid., p. 27), il n'est pas rare que l'enclitique -que soit traité comme un mot indépendant, et ce tout au long du Moyen Âge. 
Discre|tus dubi|tet, ${ }^{\mathrm{P}}$ qui | quidnam | sint bene | penset.

20Ex quo | grex gemi|tus ${ }^{\mathrm{P}}$ de|sistat | promere | sanctus.

Abbas | a pue $\mid$ ro $^{\mathrm{P}} \mathrm{Vi} \mid$ talis | se dare | Christo

Certe | disposu|it, ${ }^{\mathrm{P}}$ quod | post haud | segniter | egit.

Ni pro|sunt lacri|me. ${ }^{\mathrm{P}}$ Quid e|is revo|catur ad|esse?

Cui brevi|tas vi|te ${ }^{\mathrm{P}}$ pre|sentis | possit ab|esse?

25Ex prece $\mid$ multimo $\mid$ da $^{\mathrm{P}}$ tribu|untur $\mid$ regna be|ata,

Devo|teque da|tis, ${ }^{\mathrm{P}}$ ut | res est, | ex ali|mentis,

Ecclesi|e ma|tris ${ }^{\mathrm{P}}$ in | pauperi|bus recre|andis,

Aut u|troque mo $\mid$ do $^{\mathrm{P}}$ redi|tus dis|ponitur | ordo

Ad Chris|tum, ${ }^{\mathrm{T}}$ qui | dirigat $\mid$ hunc ${ }^{\mathrm{H}}$ ad $\mid$ regna po|lorum. Amen.

Rouen (cathédrale N.-D.), no 122, 15, p. 532

T. Sancte Marie Rothomagensis ecclesie

1 Abbas | Vita|lis, ${ }^{\mathrm{P}}$ qui | vitam | semper a|mavit,

Vivat et $\mid$ eter|na ${ }^{\mathrm{D}} \mid$ luce fru|atur. $\mathrm{A} \mid$ men.

Item

1Que lex $\mid$ carna $\mid$ lis $^{\mathrm{P}}$ sit, $\mid$ cuius $\mid$ conditi|onis,

Mors ar|gumen|tis ${ }^{\mathrm{P}}$ propiat $^{60} \mid$ horren|de rati|onis.

Abbas | Vita|lis ${ }^{\mathrm{P}}$ sanc|tus, pius | atque be|nignus,

En mori|tur, vi|te ${ }^{\mathrm{P}}$ meli|oris munere $\mid$ dignus.

5Dumque quod | est ter|re ${ }^{\mathrm{P}}$ sol|vit, dum | carnea | castra

Exit, ha|bet ce|lum, ${ }^{\mathrm{P}}$ ce|li posi|tus super $\mid$ astra.

Rouen (Saint-Ouen), n' 122, 17, p. 533

T. Sancti Petri Sanctique Audoeni Rothomagi

1 Abbas | Vita|lis, ${ }^{\mathrm{P}}$ vir|tute ni|tentibus | alis,

Carne, quod $\mid$ opta|vit, ${ }^{\mathrm{P}}$ reso|lutus, ad | astra vo|lavit.

Vixit in $\mid$ hac vi|ta ${ }^{\mathrm{P}}$ sanc|tissimus $\mid$ hic here $\mid$ mita,

Et pius | et le|nis, ${ }^{\mathrm{P}}$ par|cus sibi, | largus e|genis.

60. L'édition Dufour donne le texte propriat, insatisfaisant métriquement (-priat peut difficilement se réduire à une seule syllabe) et grammaticalement (le verbe proprio est transitif); je corrige donc en propiat («la mort s'approche avec des ruses d'une horrible sorte»). Dans l'édition de Léopold Delisle (Delisle (éd.), 1866, p. 291) le texte est parat, compatible métriquement, mais non grammaticalement (on attendrait parat argumenta). 
5Iste po|testa|tes ${ }^{\mathrm{P}}$ et $\mid$ mundi $\mid$ prosperi|tates

Non at $\mid$ tende $\mid$ bat, ${ }^{\mathrm{P}}$ mala $\mid$ damnans $\mid$ sancta do|cebat;

Sic Chris|to ve|re ${ }^{\mathrm{P}}$ so|li cupi|endo pla|cere,

Fortis | cum mun $\mid$ do $^{\mathrm{P}}$ pu|gnavit | fine se|cundo.

Hinc est | gauden|dum, ${ }^{\mathrm{P}}$ non | pro pas|tore do|lendum,

10Qui supe|rans se|vum ${ }^{\mathrm{P}}$ pre|donem | vivet in | evum.

Quod con $\mid$ cedat e $\left|\mathbf{i}^{\mathrm{D}}\right|$ gracia $\mid$ magna De $\mid \mathbf{i}$. Amen.

Rouen (Saint-Paul), no 122, 20, p. 534

Titulus Sancti Pauli Rothomagi

1Quisquis | celes $\mid$ ti $^{\mathrm{P}}$ vult $\mid$ esse be|atus in | aula,

Huius ad $\mid$ exem $\mid$ plum $^{\mathrm{D}}$ | vivat in $\mid$ orbe pa|tris.

Iste bo|nus can|tor, ${ }^{\mathrm{P}}$ pas|tor pius, | optimus | abbas,

Facun|dus, sapi|ens, ${ }^{\mathrm{D}}$ | religi|osus e|rat.

\section{Angleterre}

Gloucester (S. Peter), no 122, 83, p. 554-555

\section{T. Sancti Petri de Gloecestra}

Cuius ad $\mid$ exa $\mid$ men $^{\mathrm{P}}$ dat $\mid$ gratia $\mid$ sola iu|vamen,

$\mathrm{Nec}$ pro|sunt meri|ta ${ }^{\mathrm{P}}$ ius|tis, pie|tate re|mota,

Rex pie, | Vita|lis ${ }^{\mathrm{P}}$ sis | crimini|bus veni|alis.

Sunt bona $\mid$ nota ti|bi, ${ }^{\mathrm{D}}$ | non peri|tura si|bi.

5 At mise $\mid \operatorname{ros}$ il| $\operatorname{los}^{\mathrm{P}}$ men|tis rati|one pu|sillos,

Funera $\mid$ qui ri|dent, ${ }^{\mathrm{D}} \mid$ nec sua $\mid$ fata vi|dent.

Corrige | clemen|ter, ${ }^{\mathrm{P}}$ de|linquunt | nanque sci|enter.

Fac tepe $\mid$ at rati|$\left|\mathbf{o},{ }^{\mathrm{D}}\right|$ desipi|ens viti|o.

Casibus | indul|tis ${ }^{\mathrm{P}}$ mise|rere qui|busque se|pultis:

10 Nec sint, | Christe, re $\mid \mathbf{i}^{\mathrm{D}}{ }^{\mathrm{D}}$ quos iuvat | arra spe|i.

Evesham (S. Mary), no 122, 85, p. 555

T. Sancte Marie Eoveshammensis ecclesie

1Abbas | Vita|lis ${ }^{\mathrm{P}}$ doc|trina | spiritu|alis,

Multos | instru|xit, ${ }^{\mathrm{P}}$ plu|res de | morte re|duxit;

Vita fu|it ${ }^{\mathrm{T}}$ di|gnissima, $\mid$ sic $^{\mathrm{H}}$ est $\mid$ mors preti|osa;

Sit dia|dema ${ }^{\mathrm{F}}$ la|borum | cum sanc|tis speci|osa. Amen. 
Pershore (S. Mary), no 122, 86, p. 555

T. Sancte Marie de Persora

1 Abbas | Vita|lis ${ }^{\mathrm{p}}$ ver|bis fac|tisque be|atis,

Multos | ad sum $\mid \mathrm{mi}^{\mathrm{P}}$ pre|misit | gaudia | celi,

Quos tan|dem sequi|tur, ${ }^{\mathrm{P}}$ cum | sanctis | luce po|titur.

Abingdon (S. Mary), $\mathrm{n}^{\circ}$ 122, 101, p. 558-559

\section{T. Sancte Marie Abbendonie}

${ }^{16}{ }^{6}$ Vita bre|vis ${ }^{\mathrm{T}}$ ca|susque le|vis, ${ }^{\mathrm{H}}$ nec | spes reme|andi:

Quanta se $\mid$ res,${ }^{\mathrm{T}}$ hinc $\mid$ tanta fe $\mid$ res. ${ }^{\mathrm{H}}$ Sit $\mid$ cura pa|randi.

Plura se|ras, ${ }^{\mathrm{T}}$ ut | plura fe|ras; ${ }^{\mathrm{H}}$ ne $\mid$ non seru|isse

Penite $\mid$ at, ${ }^{\mathrm{T}}$ cum $\mid$ nil vale $\mid \mathbf{a t}^{\mathrm{H}}$ tibi $\mid$ penitu|isse.

5 Qui revo|cas ${ }^{\mathrm{T}}$ quod in $\mid$ arce lo|cas, ${ }^{\mathrm{H}}$ Petre, | iure po|tenti,

Huic ape|ri ${ }^{\mathrm{T}}$ val|vas. Supe|ri ${ }^{\mathrm{H}}$ plau|dant veni|enti.

Item alterius

1 Iustus | leta|tur, ${ }^{\mathrm{P}}$ cum | carne su|a spoli|atur.

Ordine $\mid$ nanque De $\mid i^{\mathrm{p}}$ mori|ens ori|tur requi|ei :

Sic hic | Vita|lis ${ }^{\mathrm{P}}$ pas|tor, vi|ta speci|alis,

Hinc nece $\mid$ subtrac|tus ${ }^{\mathrm{p}}$ requi|escit, | gaudia $\mid$ nactus.

Eynsham (Holy Trinity, S. Mary), no 122, 102, p. 559

T. Sancte Trinitatis et Sancte Marie Egneshamnie

1 Non con|demna|tur ${ }^{\mathrm{P}} \mathrm{cu} \mid$ ius bona | vita pro|batur.

Immo $\mid$ salva|tur ${ }^{\mathrm{P}} \mathrm{cu} \mid$ ius pia | vita pro|batur,

Abbas | Vita|lis ${ }^{\mathrm{p}}$ vir | vite | spiritu|alis.

Hic sanc|te vi|xit, ${ }^{\mathrm{P}}$ nunc $\mid$ cum Domi|no requi|escit.

Oxford (Holy Trinity, S. Frideswide), no 122,103, p. 559-560

T. Sancte Trinitatis et Sancte Fridesvide Oxinefordensis

1 Cui de|servis|ti, ${ }^{\mathrm{P}}$ Vi|talis, e| um meru|isti,

Regi $\mid$ morta|li ${ }^{\mathrm{P}}$ non, | immo | perpetu|ali,

Qui pro | plebe su|a, ${ }^{\mathrm{P}}$ que | capti|vata ma|nebat,

Quam cupi $\mid$ dus ser $\mid$ pens $^{\mathrm{p}}$ ad $\mid$ Tartara $\mid$ dira tra|hebat,

5Quatinus | eripe $\mid$ ret $^{\mathrm{P}}$ quos $\mid$ carcer $\mid$ noctis ha|bebat,

61. Vers trinini salientes (voir supra, $\mathrm{n}^{\mathrm{o}} 122,12$ ). 
Lapsus | de ce|lo, ${ }^{\mathrm{P}}$ veni|ens in | ventre pu|elle,

Figitur | in li|gno ${ }^{\mathrm{P}}$ sine $\mid$ culpa, | non sine | velle.

Hic te $\mid$ Lucife $\mid \mathrm{ri}^{\mathrm{P}}$ di|gnetur $\mid$ reddere $\mid$ selle.

Battle (S. Martin), $\mathrm{n}^{\mathrm{o}}$ 122, 146, p. 570

\section{T. Sancti Martini de Bello}

1Transit, ut | umbra, ${ }^{\mathrm{F}}$ de|cora ma|lignaque $\mid$ gloria $\mid$ mundi.

Ut quid a|mantur, | cum per|dantur, | gaudia | mundi?

Nullus | pasto|rum ${ }^{\mathrm{P}}$ rema|net nul|lusque bo|norum.

Flos a|rens fe $\mid$ num $^{\mathrm{P}}$ fit, $\mid$ carnis $\mid$ gloria $\mid$ cenum.

5Transiit | hac vi|ta ${ }^{\mathrm{P}} \mathrm{Vi} \mid$ talis $\mid$ sic here $\mid$ mita.

Dat fla|tum ce|lo, ${ }^{\mathrm{P}}$ dans | carnem | pulveris | imo.

Wilton (S. Mary, S. Edith), no 122, 153, p. 571

T. Sancte Marie Sancteque Eadithe Wiltoniensis ecclesie

Gloria | quid re|rum, ${ }^{\mathrm{P}}$ vel | quid mora | longa di|erum

Prosunt, | tam for $\mid$ tis $^{\mathrm{P}}$ cum $\mid$ sit con $\mid$ clusio $\mid$ mortis,

Ut sub | sorte pa|ri ${ }^{\mathrm{P}}$ pos $\mid$ sit cunc|tis domi|nari?

Sed des|peran $\mid$ dum $^{\mathrm{P}}$ non $\mid$ est aut $\mid$ iure do|lendum

5De nece | iusto|rum: ${ }^{\mathrm{P}}$ requi|es sit | nam Deus | horum.

Unde pi|is lacri|mis, ${ }^{\mathrm{P}}$ peti $\mid$ mus, vos $\mid$ parcite $\mid$ vestris;

Nam si | sic vi|xit, ${ }^{\mathrm{P}}$ no|bis ut $\mid$ cartula | dixit,

Vita|lis, ce $\mid i^{\mathrm{P}}$ frui|tur modo $\mid$ parte se|reni,

Quodque si|bi no $\mid$ men $^{\mathrm{P}}$ Vi|talis $\mid$ contulit $\mid$ omen,

10Hoc habet | in Chris|to: ${ }^{\mathrm{P}}$ mun|do nam | vixerat | isto

Prudens, | munifi|cus, ${ }^{\mathrm{P}}$ cas|tus, pie|tatis a|micus.

Ergo si $\mid$ bi sum $\mid \mathrm{mi}^{\mathrm{P}}$ con $\mid$ cedat $\mid$ gratia $\mid$ Christi

Ut prece $\mid$ sancto $\mid$ rum $^{\mathrm{P}}$ con|sors sit $\mid$ factus e|orum.

Que dedi|mus ves $\mid$ tris $^{\mathrm{P}}$ ea $\mid$ dem vos $\mid$ reddite $\mid$ nostris.

Bardney (S. Oswald), no 122, 166, p. 573-574

T. sancti Oswaldi regis et martyris in Bardanai

1 Miles | duxque gre|gis ${ }^{\mathrm{P}}$ gemi|ne per | pascua | legis,

Ex atri|i cu|ra ${ }^{\mathrm{P}}$ te $\mid$ post cer|tamina $\mid$ plura.

Continet $\mid$ aula De $\mid \mathbf{i}^{\mathrm{P}}$ refo|vetque si|nus requi|ei; 
Virgine|i ce|tus ${ }^{\mathrm{P}}$ ta|li dul|cedine | letus

5 Visibus | atque cho|ris ${ }^{\mathrm{P}}$ nunc $\mid$ delec|tare ca|noris.

Grex con|fesso|rum ${ }^{\mathrm{P}}$ can|dens ut | flos lili|orum

Te gau|det iun $\mid \mathbf{g i}^{\mathrm{P}}$ sibi, | te quoque | nomine | fungi

Vite, | Vita|lis, ${ }^{\mathrm{P}}$ quia | vite | perpetu|alis;

Et rose $\mid$ e gen $\mid$ tis $^{\mathrm{P}}$ ex $\mid$ ercitus | igne ca|lentis

10Omnia | magno|rum ${ }^{\mathrm{P}}$ tibi | monstrat ho|nora la|borum;

Ordo se $\mid$ nato $\mid$ rum $^{\mathrm{P}}$ te $\mid$ curia $\mid$ discipu|lorum,

Atque De $\mid$ o ple $\mid$ nus $^{\mathrm{P}}$ te $\mid$ convocat $\mid$ ordo no|venus,

Ut prius $\mid$ igno $\mid$ ta $^{\mathrm{P}}$ bona $\mid$ sumas $\mid$ ad tua $\mid$ vota,

Clamans: | En vi|vam! ${ }^{\mathrm{P}}$ De|glutio | namque ${ }^{62}$ | salviam,

15Qua novus | est sos|pes ${ }^{\mathrm{P}}$ et | vivax | celicus | hospes!

En tot | fulte bo|nis ${ }^{\mathrm{P}}$ memor | esto | conditi|onis

Prime, | nec ce $\mid \mathbf{l i}^{\mathrm{P}}$ Domi|num pro $\mid$ gente fi|deli

Cesses $\mid$ ora $\mid$ re,${ }^{\mathrm{P}}$ con|tagia $\mid$ nostra la|vare,

Moribus | orna|re, ${ }^{\mathrm{P}}$ nos | inde be|atifi|care.

20 At si | forte do $\mid$ les $^{\mathrm{P}}$ quod $\mid$ traxit $\mid$ carnea $\mid$ moles,

Concito | solva|ris ${ }^{\mathrm{P}}$ sum|maque qui|ete fru|aris.

Huic ad $\mid$ sola $\mid$ men $^{\mathrm{D}} \mid$ consonet $\mid$ omnis : A|men.

Salisbury (Cathedral S. Mary), $\mathrm{n}^{\circ} 122,186$, p. 580

T. Sancte Marie Salesberiensis ecclesie

1 Abas | Vita|lis ${ }^{\mathrm{P}}$ mona|corum | gloria | vixit;

Dum vi|xit, fe|cit ${ }^{\mathrm{P}}$ que | fecit | semper ho|neste.

Sermo De|i vacu|us ${ }^{\mathrm{P}}$ num|quam fuit $\mid$ eius in | ore.

Quod docu|it gen $\mid$ tes $^{\mathrm{P}}$ in | se prius | istud a|gebat;

5Illici|tos ac|tus ${ }^{\mathrm{P}}$ prohi|bens nun|quam faci|ebat.

Ergo De $\mid$ i pie $\mid \operatorname{tas}^{\mathrm{P}}$ et $\mid$ gratia $\mid$ det sibi | vitam

Eter|nam, mul|tis ${ }^{\mathrm{P}}$ quam | promit|tebat ha|bendam.

62. Le vers est faux, peut-être pour des raisons de transmission du texte, mais plus vraisemblablement parce que le rédacteur aura sacrifié la métrique au jeu des sonorités, organisées autour de la quadruple répétition du son - $a m$. Le fait que namque fasse du $5^{\mathrm{e}}$ pied un vers spondaïque n'a rien d'extraordinaire, ni la quantité de -que (long), si l'on se réfère à celle de ille dans le rouleau $n^{\circ} 105,132$, v. 14. En outre namque se trouve ici en $2^{\mathrm{e}}$ position après une ponctuation forte (Deglutio namque), tout comme dans le rouleau $\mathrm{n}^{\circ} 114,61$, Item, v. 2 (Tali namque modo); cette position est conforme aux usages des poètes classiques et de Tite-Live. Il me semble qu'on puisse sauver ce vers dans une production poétique médiévale où la quantité le cède devant le nombre et la couleur des syllabes. 
Pueriles versus

$1 \mathrm{O}$ flos | Vita|lis, ${ }^{\mathrm{P}}$ mona|corum | gloria, | mortem

Nocte di|eque tu|am ${ }^{\mathrm{P}}$ lu|gent mona|chique pa|rentes;

Nam pie $\mid$ tas te $\mid$ cum $^{\mathrm{P}}$ fuit $\mid$ et con|cordia, | pax; lis

Non ade|rat; Cris|tum ${ }^{\mathrm{P}}$ sem|per Vi|talis a|mabat,

5Et, dum | vivus e|ras, ${ }^{\mathrm{P}}$ Cris|to ser|vire stu|debas.

Abas | Vita|lis, ${ }^{\mathrm{P}}$ tibi | sit laus | vita per|hennis,

Nam dum | visis|ti, ${ }^{\mathrm{P}}$ ves|tes es|camque de|disti.

Pauperi|bus ius|tis ${ }^{\mathrm{P}}$ lar|gus blan|dusque fu|isti;

Sprevis $\mid \mathrm{ti}^{\mathrm{T}}$ ter $\mid$ ras $^{\mathrm{P}}$ et $\mid$ Cristum | semper a|masti.

10 Ergo | perpetu| um $^{\mathrm{P}}$ tibi $\mid$ det Cris|tus para|disum.

Cum mors $\mid$ equa|li ${ }^{\mathrm{P}}$ mor|talia $\mid$ conditi|one

Subruat, | et mor $\left|\mathrm{ti}^{\mathrm{D}}\right|$ ni super $\mid$ esse que $\mid$ at

Sic agat $\mid$ omnis ho|mo ${ }^{\mathrm{P}}$ ne | post mor|tem mori|atur.

Ne sit | pena du|plex, ${ }^{\mathrm{D}}$ | sic agat | omnis ho|mo.

15Abas | Vita|lis, ${ }^{\mathrm{P}}$ fons, | exem|plum pie|tatis,

In Cris|to vi|vens, ${ }^{\mathrm{D}}$ | non obi|turus, o|bit.

Nunc quia | natu|re ${ }^{\mathrm{P}}$ per|solvit | debita, | Cristus

Sit sibi | lumen, ho|nor, ${ }^{\mathrm{D}}$ | gloria, | vita, sa|lus.

Southwark (S. Mary), no 122, 203, p. 584

T. Sancte Marie Suthewercensis ecclesie

1 Hic quia $\mid$ doctri|na ${ }^{\mathrm{P}}$ vigu|it pius | absque ru|ina,

Et quia | perla|te ${ }^{\mathrm{P}}$ ful|sit mo|rum gravi|tate,

More pre|ces de $\mid$ mus. ${ }^{\mathrm{P}} \mathrm{Sic} \mid$ isti | subveni|emus,

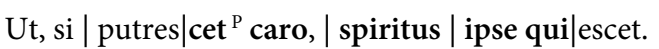

London (Cathedral S. Paul), no 122, 204, p. 585

T. sancti Paulis (sic) Londoniensis ecclesie Versus Radulfi, filii Fulcredi Cadomensis ${ }^{63}$

1Ista ne $\mid$ mo sci $\mid \operatorname{ta}^{\mathrm{P}}$ tam $\mid$ sprevit in|ercia $\mid$ vita

Mundi | terre $\mid$ na, ${ }^{\mathrm{P}}$ ten|dens al|te sua | frena,

63. À propos de Raoul de Caen, voir l'introduction. 
Iste ve|lut fe $\mid$ cit $^{\mathrm{P}} \mathrm{Vi} \mid$ talis $\mid$ nomine $\mid$ dictus.

Se non | inie $\mid$ cit $^{\mathrm{P}}$ mor|ti, ${ }^{\mathrm{H}}$ car|nalibus | ictus.

5Omnia | pessima | sub necis | infima | missa me|avit.

Cordis et $\mid$ intima $\mid$ traxit $^{\mathrm{P}}$ ad $\mid$ optima $\mid$ falsaque $\mid$ cavit.

Novit et | ipse ma|lum, ${ }^{\mathrm{P}}$ nec ad $\mid$ effec|tum mala $\mid$ duxit.

Quod sciit | imple $\mid$ vit $^{\mathrm{D}}$ |; novit et | ipse ma|lum.

Constitit | immobi|lis ${ }^{\mathrm{P}}$ con|tra flam|mas vici|orum.

10Hic nequi|it flec|ti ; $^{\mathrm{D}}$ constitit | immobi|lis.

Nulli | terre|ne ${ }^{\mathrm{P}}$ fuit | huius | vita se|cunda.

Supposi|tos pe $\mid \mathrm{ne}^{\mathrm{P}}$ plu|res velut $\mid$ ex maris | unda

Erep|sit ple|ne ${ }^{\mathrm{P}}$ mons|trans non $\mid$ pretere|unda.

Transiit $\mid$ obsce $\mid \mathrm{ne}^{\mathrm{P}}$ cum $\mid$ creduli|tate ro|tunda

15Vite | ferven $\mid$ tes $^{\mathrm{P}}$ fluc|tus. ${ }^{\mathrm{H}}$ Modo $\mid$ sede io|cunda

Fit resi|dens. ${ }^{\mathrm{T}}$ Er $\mid$ go $^{\mathrm{P}}$ data $\mid$ sit sibi $\mid$ tanta po|testas,

Ut nos | a ter|go ${ }^{\mathrm{P}}$ sump $\mid$ tos $^{\mathrm{H}}$ trahat $\mid$ eius ho|nestas.

Flos ab|batum, | fons ${ }^{\mathrm{P}}$ boni|tatum, | vita po|tentum,

Imperi|um ${ }^{\mathrm{T}}$ re|gum, ${ }^{\mathrm{P}}$ scep|trum ${ }^{\mathrm{H}}$ le|gum, sapi|entum

20 Pax et ho|nor, ${ }^{\mathrm{T}}$ spes, | rex, ${ }^{\mathrm{P}}$ lex, | dux, ${ }^{\mathrm{H}}$ lux, | gloria, | virtus,

Corruit, | adnichi|latur. Quid meru|it mori|atur?

Ergo $\mid$ mors is $\mid \mathrm{ti}^{\mathrm{P}}$ suc|currat $\mid$ passio $\mid$ Christi;

Huic Deus | inde pi| $\mathrm{e}^{\mathrm{P}}$ suc|currant | vota Ma|rie.

Filius | unicus $\mid$ et ${ }^{\mathrm{D}}$ | Spiritus $\mid$ almus a|lius

25Salvifi|cet ${ }^{\mathrm{T}}$ nos, | purifi|cet ${ }^{\mathrm{H}}$ nos, | iustifi|cet nos.

\section{Bibliographie}

Bède LE VÉnÉrAble, Bedae venerabilis opera, t. 1, Opera didascalica: De orthographia, De arte metrica et de schematibus et tropis, De natura rerum, Turnhout, Brepols (Corpus christianorum. Series Latina, $\mathrm{n}^{\circ}$ 123A), 1975.

Baldricus Burgulianus [Baudri de Bourgueil], Carmina, t. 1, trad. et éd. Jean-Yves Tilliette, Paris, Les Belles-Lettres (Auteurs latins du Moyen Âge), 1998.

Delisle, Léopold (éd.), Rouleaux des morts du $I X^{e}$ au $X V^{e}$ siècle, Paris, Renouard (Publications pour la Société de l'histoire de France, no ${ }^{135}$ ), 1866.

Dufour, Jean, «Le rouleau des morts de saint Bruno», in Comptes rendus des séances de l'Académie des Inscriptions et Belles-Lettres de l'année 2003, janvier-mars, Paris, Académie des Inscriptions et Belles-Lettres, 2003, p. 5-26. 
Dufour, Jean (éd.), Recueil des rouleaux des morts (VIII siècle-vers 1536), Jean FAVIER (dir.), Paris, Académie des Inscriptions et Belles-Lettres (Recueil des historiens de la France. Obituaires. Série in-4, $\mathrm{n}^{\circ}$ 8) 2005-2013, 5 vol.

Goullet, Monique, «Poésie et mémoire des morts. Le rouleau funèbre de Mathilde, abbesse de la Sainte-Trinité de Caen (†1113)», in Jean-François Cottier, Martin Gravel et Sébastien Rossignol (dir.), 'Ad libros'. Mélanges offerts à Denise Angers et Joseph-Claude Poulin, Montréal, Presses de l'Université de Montréal, 2010, p. 163-198.

Norberg, Dag., Introduction à l'étude de la versification latine médiévale, Stockholm, Almqvis et Wiksell (Studia latina Stockholmiensia, no ${ }^{\circ}$ ), 1958.

\section{Annexe}

Le rouleau de Vital de Savigny a été partiellement conservé dans sa forme originale (voir J. Dufour, vol. 1, p. 514-515). Les Archives nationales le conservent sous la cote AE/II/138 ; l'original n'est pas accessible, mais la base Archim (http:// www.culture.gouv.fr/documentation/archim/accueil.html) permet de voir les quinze feuilles de parchemin assemblées entre elles. Ghislain Brunel, conservateur aux Archives nationales, a mis gracieusement à notre disposition les images de ce rouleau; nous avons choisi de publier les cinq dernières (images 25 à 29, correspondant aux figures 1 à 5 ci-dessous), où l'on trouvera les titres (tituli) et les poèmes. L'intérêt de ces rouleaux est multiple, tant par le contenu que par la variété des écritures, la mise en page, la décoration, l'utilisation des couleurs.

Figure 1: titres non versifiés: $n^{\circ} 181-185$ et 187-188; titre versifié: ${ }^{\circ} 186$.

Figure 2: titres non versifiés: $n^{\circ}$ 189-196. Remarquer le dessin, fruste et sans décor, d'un homme portant sur ses épaules un hybride zoomorphe à forme d'animal fantastique (tête et cou de "dragon », ailes et plumage d'oiseau, longue queue fourchue), associé ici à une figure humaine, qui trace avec son corps (vertical) et son fardeau (horizontal) le T du titulus de S. Mary et S. Botolph. Mes remerciements à Thierry Buquet (CRAHAM-UMR 6273).

Figure 3: titres non versifiés: $n^{\circ}$ 197-202; toutefois dans le titre $\mathrm{n}^{\circ} 202$, après Amen, suit un distique (Vita beatarum Deus et requies animarum / Vita Vitali det vivere perpetuali); titres versifiés: $\mathrm{n}^{\circ} 203$ et les trois premiers vers du titre $\mathrm{n}^{\circ}$ 204; en face du début du poème on lit d'une autre main: versus Radulfi, filii Fulcredi Cadomensis (voir l'article, note 7).

Figure 4: suite du poème $n^{\circ} 204$; les autres titres ( $\left.n^{\circ} 205-208\right)$ ne sont pas versifiés.

Figure 5: c'est un verso resté vierge, où on peut lire par transparence les titres $\mathrm{n}^{\circ} 83-95$ du recto (image 13 du rouleau de Vital, dans la base Archim). 


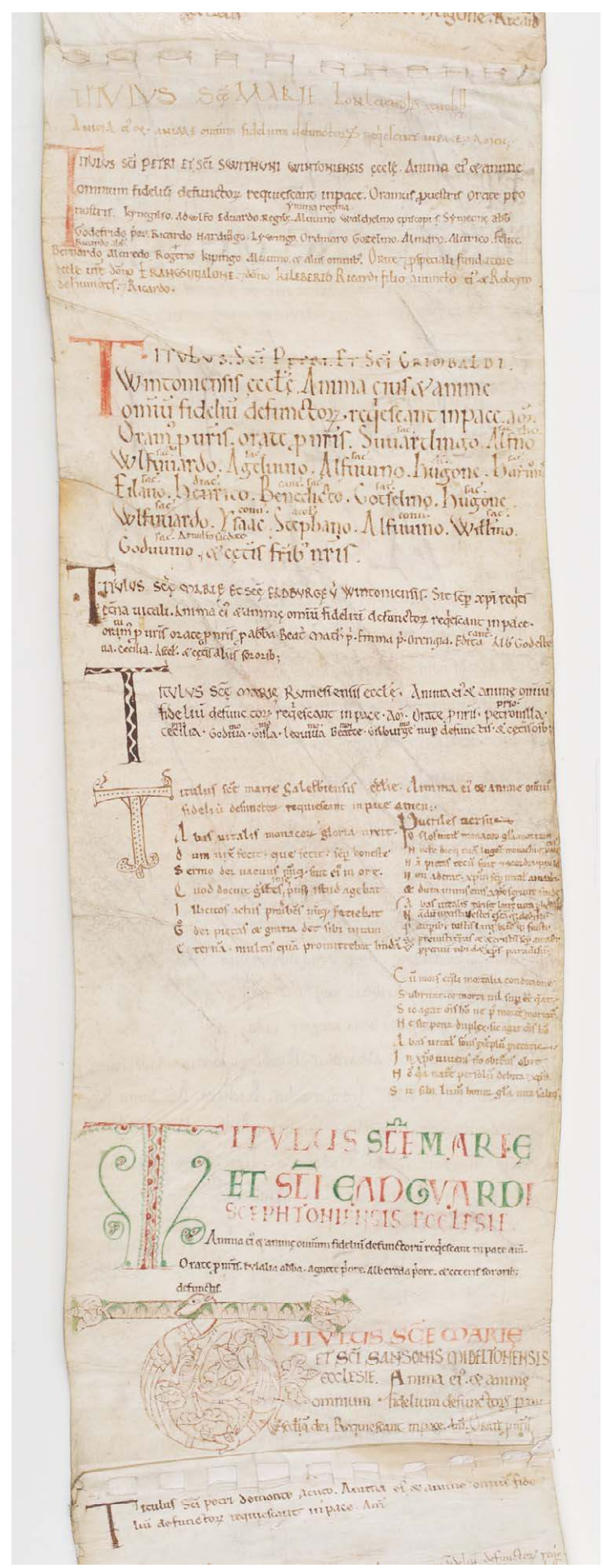

Figure 1 


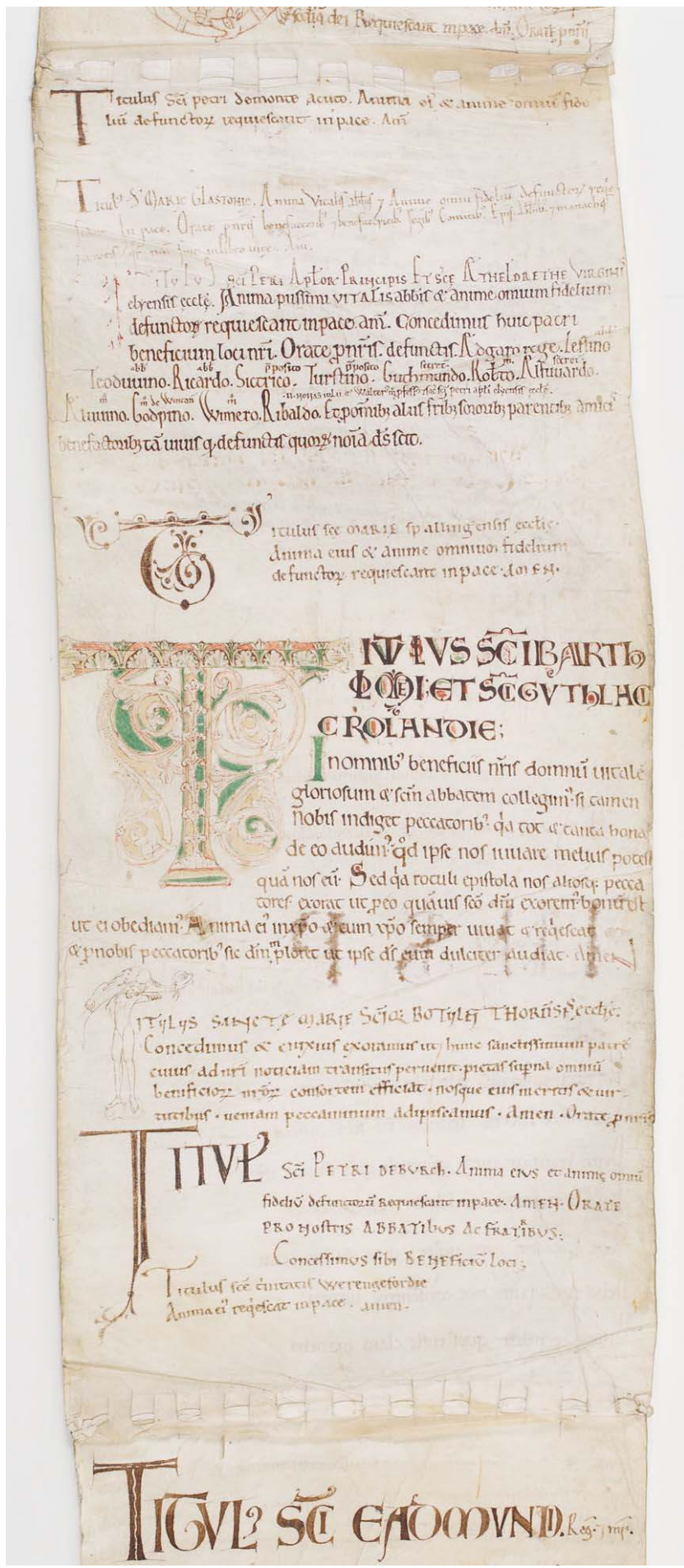

Figure 2

Tabularia «Études», $\mathrm{n}^{\circ}$ 16, 2016, p. 217-278, 20 octobre 2016 


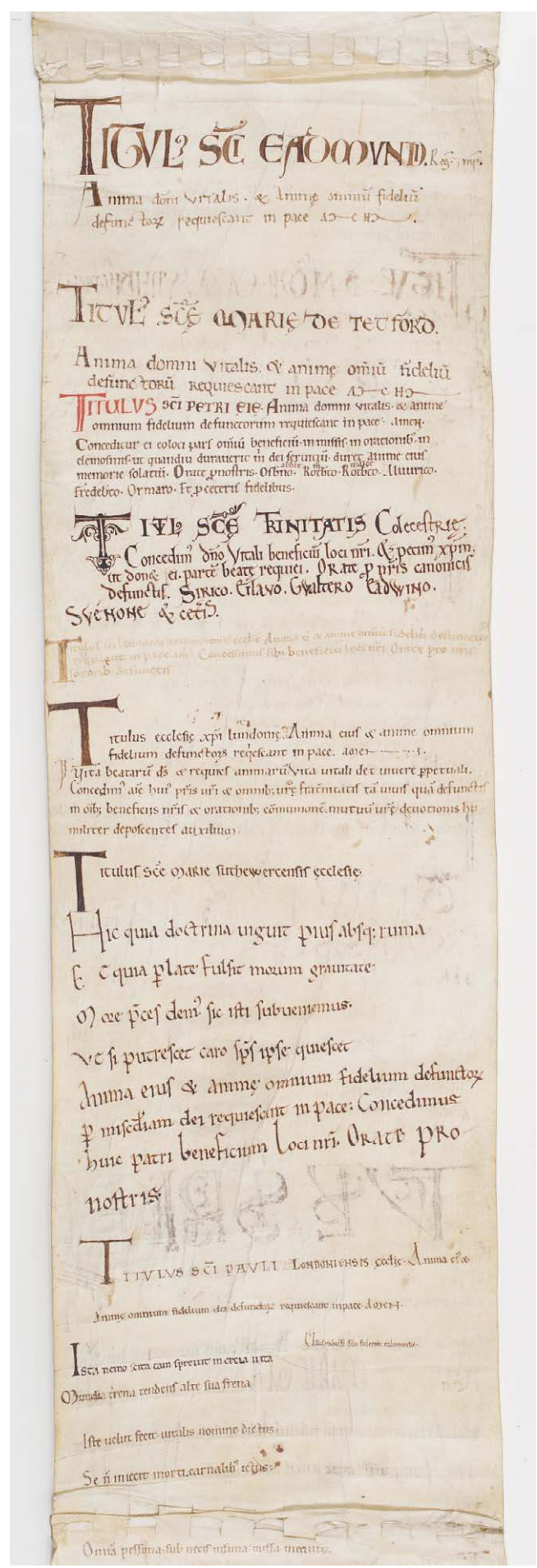

Figure 3 


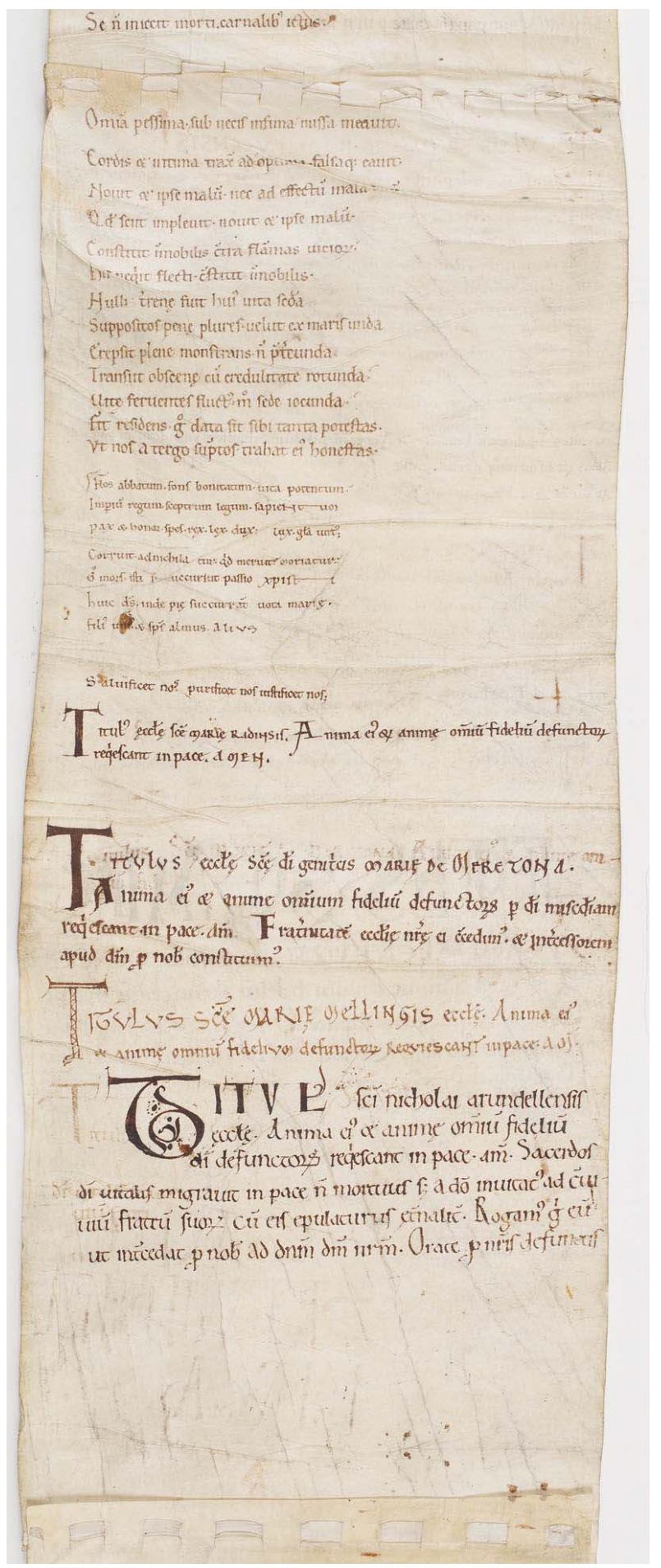

Figure 4

Tabularia «Études», $\mathrm{n}^{\circ}$ 16, 2016, p. 217-278, 20 octobre 2016 


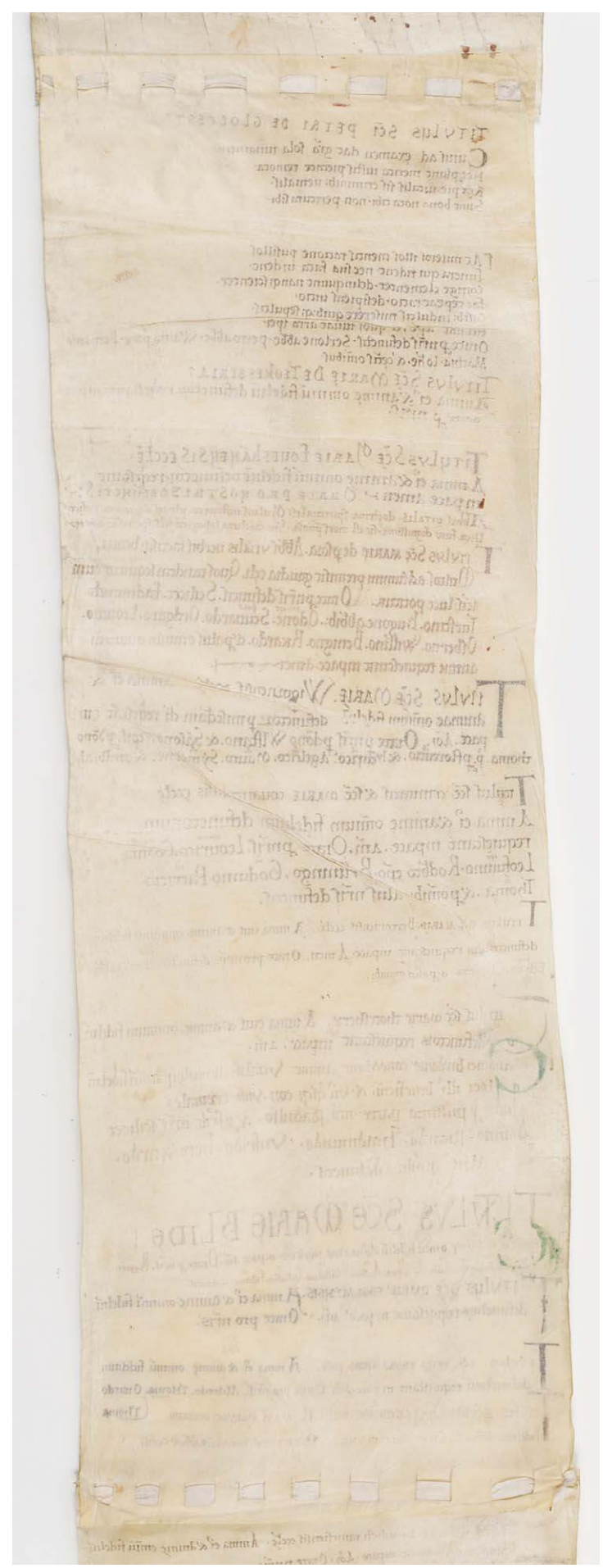

Figure 5

http://www.unicaen.fr/mrsh/craham/revue/tabularia/print.php?dossier=dossier15\&file=03goullet.xml 